Board of Governors of the Federal Reserve System

International Finance Discussion Papers

Number 1310

February 2021

\title{
Globalization, Trade Imbalances and Labor Market Adjustment
}

Rafael Dix-Carneiro, Joao Paulo Pessoa, Ricardo Reyes-Heroles, Sharon Traiberman

\begin{abstract}
Please cite this paper as:
Dix-Carneiro, Rafael, Joao Paulo Pessoa, Ricardo Reyes-Heroles, Sharon Traiberman (2021).

"Globalization, Trade Imbalances and Labor Market Adjustment," International Finance Discussion Papers 1310. Washington: Board of Governors of the Federal Reserve System, https://doi.org/10.17016/IFDP.2021.1310.
\end{abstract}

NOTE: International Finance Discussion Papers (IFDPs) are preliminary materials circulated to stimulate discussion and critical comment. The analysis and conclusions set forth are those of the authors and do not indicate concurrence by other members of the research staff or the Board of Governors. References in publications to the International Finance Discussion Papers Series (other than acknowledgement) should be cleared with the author(s) to protect the tentative character of these papers. Recent IFDPs are available on the Web at www.federalreserve.gov/pubs/ifdp/. This paper can be downloaded without charge from the Social Science Research Network electronic library at www.ssrn.com. 


\title{
GLOBALIZATION, TRADE IMBALANCES AND LABOR MARKET ADJUSTMENT*
}

\author{
Rafael Dix-Carneiro, Duke University and NBER \\ João Paulo Pessoa, São Paulo School of Economics \\ Ricardo Reyes-Heroles, Federal Reserve Board \\ Sharon Traiberman, New York University
}

February 2, 2021

\begin{abstract}
We study the role of global trade imbalances in shaping the adjustment dynamics in response to trade shocks. We build and estimate a general equilibrium, multi-country, multi-sector model of trade with two key ingredients: (a) Consumption-saving decisions in each country commanded by representative households, leading to endogenous trade imbalances; (b) labor market frictions across and within sectors, leading to unemployment dynamics and sluggish transitions to shocks. We use the estimated model to study the behavior of labor markets in response to globalization shocks, including shocks to technology, trade costs, and inter-temporal preferences (savings gluts). We find that modeling trade imbalances changes both qualitatively and quantitatively the short- and long-run implications of globalization shocks for labor reallocation and unemployment dynamics. In a series of empirical applications, we study the labor market effects of shocks accrued to the global economy, their implications for the gains from trade, and we revisit the "China Shock" through the lens of our model. We show that the US enjoys a $2.2 \%$ gain in response to globalization shocks. These gains would have been $73 \%$ larger in the absence of the global savings glut, but they would have been $40 \%$ smaller in a balanced-trade world.
\end{abstract}

JEL Codes: F1, F16

Keywords: Globalization, labor markets, trade imbalances

\footnotetext{
*Dix-Carneiro: rafael.dix.carneiro@duke.edu; Pessoa: joao.pessoa@fgv.br; Reyes-Heroles: ricardo.m.reyesheroles@frb.gov; Traiberman: sharon.traiberman@nyu.edu. We would like to thank seminar and conference participants for helpful feedback. Traiberman gratefully acknowledges support by Early Career Research Grant 18-156-15 from the W.E. Upjohn Institute for Employment Research. The views in this paper are solely the responsibility of the authors and should not be interpreted as reflecting the views of the Board of Governors of the Federal Reserve System or of any other person associated with the Federal Reserve System.
} 
"One major contrast between most economic analyses of globalization's impact and those of the broader public ... is the focus, or lack thereof, on trade imbalances. The public tends to see trade surpluses or deficits as determining winners and losers; the general equilibrium trade models that underlay the 1990s' consensus gave no role to trade imbalances at all. The economists' approach is almost certainly right for the long run ... Yet in the long run we are all dead, and rapid changes in trade balances can cause serious problems of adjustment ..."

Paul Krugman, "Globalization: What Did We Miss?"1

\section{Introduction}

A large body of evidence shows that globalization can lead to significant labor market disruption. For instance, Autor et al. (2013) show that American workers in regions facing steeper import competition from China are less likely to work in manufacturing and more likely to be unemployed. $^{2}$ This work has generated considerable interest and research in understanding, modeling, and quantifying the adjustment process in response to globalization shocks. ${ }^{3}$ Yet, this literature has abstracted from modeling trade imbalances, and has been silent on how they could influence the labor market adjustment process.

This gap is puzzling in light of the size and persistence of trade imbalances in the last three decades, coupled with an increased discomfort among American policy makers towards trade deficits. Indeed, there is a pervasive concern among many policy makers and the public that trade deficits are undesirable as they crowd out domestic production and are detrimental to jobs and workers. ${ }^{4}$ When trade is balanced, equilibrium forces ensure that a contraction of importcompeting sectors is met with a simultaneous expansion of export-oriented sectors. On the other hand, if globalization shocks induce countries to run trade imbalances, these shifts are no longer synchronized, affecting the dynamics of reallocation. Hence, the behavior of trade imbalances can influence the dynamics of job losses and gains, especially in the presence of unemployment and labor market frictions.

In this paper, we study how endogenizing trade imbalances influences the labor market adjustment process in response to globalization. Does ignoring trade imbalances when we investigate the labor market consequences of trade shocks matter at all? How much insight do we lose in doing so? To address these questions, we build on existing models of globalization and labor market adjustment and develop an estimable, general equilibrium, multi-country, multi-sector model with three

\footnotetext{
${ }^{1}$ See Krugman (2019).

${ }^{2}$ Other recent papers tying globalization shocks to labor market disruptions include Dix-Carneiro and Kovak (2017, 2019), Pierce and Schott (2016), Costa et al. (2016), Dauth et al. (2018), Utar (2018), among many others.

${ }^{3}$ See Artuç et al. (2010), Dix-Carneiro (2014), Traiberman (2019), and Caliendo et al. (2019).

${ }^{4}$ For examples of recent policy discussions, see Scott (1998), Bernanke (2005), and Navarro (2019).
} 
key ingredients: (i) Consumption-saving decisions in each country are determined by the optimizing behavior of representative households, leading to endogenous trade imbalances; (ii) Labor market frictions across and within sectors lead to unemployment dynamics, and sluggish transitions to shocks; and (iii) Ricardian comparative advantage forces promote trade but geographical barriers inhibit it.

In our model, trade imbalances arise from country-level representative households making consumption and savings decisions. ${ }^{5}$ These decisions give rise to an Euler Equation that dictates how countries smooth consumption over time in response to shocks in productivity, trade costs, and inter-temporal preferences. Our approach relies neither on ad hoc rules for imbalances nor on specifying the path of imbalances exogenously, which are common in the international trade literature. Instead, our perspective builds on the workhorse model of imbalances in international macroeconomics, providing a natural benchmark for understanding how they shape the labor market adjustment process. ${ }^{6}$

Turning to production and the labor market, workers in each country are organized into the representative households. They choose in which sector to work, taking into account how their choices affect the household's maximizing problem. Similarly, firms choose in which sector to produce, maximizing expected discounted profits. Together, a firm and worker produce tradable intermediate varieties that are aggregated into sector-level outputs used as inputs into production, or for consumption. Goods markets are perfectly competitive, but international trade is subject to trade costs. Labor markets feature two sources of frictions: (i) switching costs to moving across sectors à la Artuç et al. (2010); and (ii) matching frictions within sectors à la Mortensen and Pissarides (1994). In particular, our framework allows for job creation and destruction to respond to trade shocks, leading to rich unemployment dynamics and speaking to a key concern of the public's anxiety over globalization. ${ }^{7}$

We estimate our model using a simulated method of moments and data from the World Input Output Database and the United States Current Population Survey. To ensure tractability of the estimation procedure, we assume the economy is in steady state and we match data moments in year 2000. The procedure conditions on the observed trade shares and allows us to estimate our parameters country by country, greatly simplifying the process.

To understand the main mechanisms in our model, we first compare its response to different sets of shocks to the response of the same model under balanced trade - an assumption that most of the trade literature makes. ${ }^{8}$ We find that modeling imbalances can lead to significantly larger

\footnotetext{
${ }^{5}$ See Obstfeld and Rogoff (1995) for a survey of this approach to imbalances in international macroeconomics.

${ }^{6}$ More recent work on global imbalances builds on the standard consumption savings model by adding financial frictions (e.g., Caballero et al. (2008) and Mendoza et al. (2009)), or demographics (e.g., Barany et al. (2018)) .

${ }^{7}$ Pavcnik (2017) reviews survey data showing that only $20 \%$ of Americans believe trade creates jobs, while $50 \%$ believe it destroys them.

${ }^{8}$ Specifically, under balanced trade, we impose that aggregate expenditure equals aggregate revenues for each
} 
unemployment and reallocation responses to globalization shocks. To be specific, consider a hypothetical positive temporary shock to Chinese productivity. In this case, consumption smoothing leads to opposing patterns of reallocation in both the short and long run. In the short run, China saves by lending to the rest of the world, and running a trade surplus. To do so, China expands its tradable sectors while other countries contract theirs, instead expanding in nontradables. In the long run, other countries pay off their debt to China, permanently expanding their tradable sectors above their initial steady-state levels. In contrast, in a balanced-trade world, short-run reallocation is only dictated by changes in comparative advantage, while a temporary shock has no long-run effects. Because it takes time for workers to find jobs, and it takes time for firms to find workers, a magnification of reallocation induces a larger response in unemployment. Importantly, we find no systematic relationship between the response of unemployment and the sign of trade imbalances.

When balanced trade is imposed, long-run allocations depend only on the final level of the shocks. On the other hand, we find that the full path of shocks matters for long-run allocations when trade imbalances are modeled. This dependence on the full path of shocks motivates us to conduct an empirical analysis where we extract the various technology, trade cost, and intertemporal preference shocks that the global economy faced between 2000 and 2014. We use the extracted shocks to answer three counterfactual questions. First, we show that the differences in predictions between our model relative to a world of balanced trade are quantitatively important in response to our extracted shocks.

In our second counterfactual exercise, we study the implications of trade imbalances and labor market frictions for the gains from trade, typically computed using the sufficient-statistics approach of Arkolakis et al. (2012) and extended by Costinot and Rodríguez-Clare (2014). Differences in the predicted consumption effects of trade are significant, both qualitatively and quantitatively. We show that imbalances and labor market frictions both play important roles in explaining the discrepancies. Relatedly, we compute the globalization gains accrued to each country between 2000 and 2014, and compare them to those obtained in a world without the global savings glut, or in a world with balanced trade. We show that the US enjoys a $2.2 \%$ gain in response to globalization shocks accrued to the world between 2000 and 2014. These gains would have been $73 \%$ larger in the absence of the global savings glut, but would have been $40 \%$ smaller if we had lived in a balanced-trade world.

Finally, we use our model to revisit the "China shock," which consists of: (i) the rapid increase in Chinese manufacturing productivity since 2000; (ii) changes in trade costs over the same time period; (iii) China's large national savings rate (the "savings glut"). ${ }^{9}$ Aligned with previous findings, we corroborate that China contributed to the decline of the United States manufacturing sectors

country in every time period.

${ }^{9}$ See Autor et al. (2016) and references therein for more discussion of the China shock. 
between 2000 and 2014. However, this decline in manufacturing was quickly accompanied by job creation in Services and Agriculture, leading to small unemployment effects. We find that shocks to the Chinese economy had a modest effect on the US trade deficit, highlighting that the evolution of the US trade deficit is a result of the full constellation of shocks hitting the global economy. On the other hand, if we feed the model with all empirically-extracted shocks but neutralize the Chinese savings glut (China's inter-temporal preference shocks), the trade deficit in the US would have shrunk in the short run, but would have been amplified in the long run. This amplification results in an even larger long-run contraction in manufacturing.

Our paper speaks to a large literature that investigates the labor market consequences of globalization, both empirically and quantitatively. We make two contributions to this literature by incorporating both involuntary unemployment and trade imbalances into the state-of-the-art Ricardian trade model of Caliendo and Parro (2015). Broadly speaking, quantitative trade models based on Eaton and Kortum (2002) have only allowed for a non-employment option (i.e., voluntary unemployment) or have focused on steady-state analyses, ignoring transitional dynamics. Caliendo et al. (2019) is an important example of a dynamic quantitative trade model in which workers make a labor supply decision and face mobility frictions across sectors and regions. However, their model does not feature job losses and unemployment. On the other end, Carrère et al. (2020) and Guner et al. (2020) incorporate search frictions and unemployment into multi-sector extensions of Eaton and Kortum (2002), but do not study out-of-steady-state dynamics. In a recent exception, Rodriguez-Clare et al. (2020) incorporates wage rigidity into the model of Caliendo et al. (2019) to investigate the unemployment effects of the China Shock on local labor markets in the United States. ${ }^{10}$

Importantly, though, none of these papers model trade imbalances. We do so by incorporating the workhorse model of imbalances used in the international macroeconomics literature allowing for savings decisions by means of an international bonds market as in Reyes-Heroles (2016). ${ }^{11}$ In that regard, our paper is closely related to Kehoe et al. (2018) who explore the implications of the increase in the United States trade deficit for the secular decline in manufacturing labor over the last four decades. However, this paper does not incorporate sluggish labor market adjustment nor unemployment dynamics. ${ }^{12}$

\footnotetext{
${ }^{10}$ In addition to these papers based on the Eaton and Kortum model, Helpman and Itskhoki (2010) add labor market frictions to a two-country Melitz model, and Heid and Larch (2016) add labor market frictions to an Armington model of trade. Coşar et al. (2016) incorporate search frictions and unemployment to an estimable small open economy Melitz model with firm dynamics, but focus on steady-state analyses. Ruggieri (2019) extends that model to study the transition in response to trade shocks. Similarly, Helpman and Itskhoki (2015) also analyze the dynamic behavior of a two-country Melitz model with labor market frictions. Finally, Kambourov (2009), Artuç et al. (2010), Dix-Carneiro (2014), and Traiberman (2019) also study transitional dynamics, but from the lenses of small open economy models.

${ }^{11} \mathrm{~A}$ few papers have analyzed the consequences of current account rebalancing on labor reallocation and unemployment by considering changes in imbalances as exogenous, e.g. Obstfeld and Rogoff (2005), Dekle et al. (2007), and Eaton et al. (2013).

${ }^{12}$ In International Macroeconomics, Kehoe and Ruhl (2009), Meza and Urrutia (2011), and Ju et al. (2014) are
} 
The rest of this paper is structured as follows. Section 2 outlines our model. Section 3 describes the data we use and our estimation procedure. In Section 4 we present our estimates and model fit analysis, but we also interpret the key mechanisms of the model by simulating a series of impulse response functions. Section 5 conducts a series of empirical applications of our model. We conclude and discuss future research in Section 6.

\section{Model}

Our model builds on existing workhorse models of globalization, trade imbalances and labor market adjustment. Trade imbalances are modeled according to the inter-temporal approach of Obstfeld and Rogoff (1995), and the trade bloc is based on Caliendo and Parro (2015). We adopt the framework in Artuç et al. (2010) to model labor mobility frictions across sectors and the structure in Mortensen and Pissarides (1994) to model search frictions and job creation and destruction. Sections 2.1 through 2.9 formalize our model showing how these different frameworks fit together.

\subsection{Environment}

There is no aggregate uncertainty, so that all agents have perfect foresight of aggregate variables. There are $i=1, \ldots, N$ countries. Each country $i$ has a constant labor force given by $\bar{L}_{i}$ workers/consumers. There are $k=1, \ldots, K$ sectors. Each sector $k$ is characterized by a continuum set of varieties $j \in[0,1]$ that can be traded across countries. These varieties are then aggregated by perfectly competitive domestic firms, in each country, into non-tradable composite sector-specific intermediate goods according to:

$$
Q_{k, i}^{I, t}=\left(\int_{0}^{1}\left(Q_{k, i}^{t}(j)\right)^{\varepsilon} d j\right)^{\frac{1}{\varepsilon}}
$$

where $Q_{k, i}^{t}(j)$ is the quantity employed of variety $j$ in sector $k$ and country $i$ at time $t$, and $\sigma=\frac{1}{1-\varepsilon}$ is the elasticity of substitution across varieties within sectors. These composite sector-specific goods are solely used as intermediate inputs for the production of a final good or for the production of varieties. The price of one unit of the composite good of sector $k$ in country $i$ is given by the price index associated with (1), which we denote by $P_{k, i}^{I, t}$.

A non-tradable final good is produced by perfectly competitive firms that aggregate sectorspecific composite goods $Q_{k, i}^{I, t}$ according to:

$$
Q_{i}^{F, t}=\prod_{k=1}^{K}\left(Q_{k, i}^{I, t}\right)^{\mu_{k, i}}
$$

examples of the scarce work studying the interaction between the current account and labor market reallocation. 
where $\mu_{k, i}>0$ and $\sum_{k=1}^{K} \mu_{k, i}=1 \forall i$. The price of one unit of the final good is given by the price index associated with (2), which we denote by $P_{i}^{F, t}$.

\subsection{Labor Markets}

Workers and single-worker firms producing varieties engage in a costly search process. Firms post vacancies, but not all of them are filled. Workers search for a job, but not all of them are successful, leading to involuntary unemployment. Each variety $j$ constitutes a different labor market. More precisely, the unemployment rate $u_{k, i}^{t}(j)$ is variety-specific, as is the vacancy rate $v_{k, i}^{t}(j)$. Both variables are expressed as a fraction of the labor force $L_{k, i}^{t}(j)$, measured as the sum of workers who are employed or unemployed and searching within sector $k$ and variety $j$ at time $t$. We impose that $m_{i}\left(u_{k, i}^{t}(j), v_{k, i}^{t}(j)\right)$ matches are formed (as a fraction of the labor force $L_{k, i}^{t}(j)$ ), where the matching function $m_{i}$ is increasing in both arguments, concave, and homogeneous of degree 1.

From now on, we drop the index $j$, but the reader should keep in mind that all labor market variables are country-sector-variety specific. Define labor market tightness as:

$$
\theta_{k, i}^{t} \equiv \frac{v_{k, i}^{t}}{u_{k, i}^{t}}
$$

The fact that $m_{i}$ has constant returns to scale allows us to write the probability that a vacancy matches with a worker as $q_{i}\left(\theta_{k, i}^{t}\right)$, for a decreasing function $q_{i}$, and the probability that an unemployed worker matches with a vacancy as $\theta_{k, i}^{t} q_{i}\left(\theta_{k, i}^{t}\right)$. We assume that workers can costlessly move across varieties $j$ within a sector, but that they face mobility costs across sectors, as we describe in the next section.

\subsection{Households}

Countries are organized into representative families, each with a household head that, taking prices as given, determines consumption, savings, and the allocation of workers across sectors maximizing aggregate utility. We first describe the utility of individual workers, then we show how household heads aggregate members' utilities. For ease of notation, we temporarily omit the country subscript $i$ and let $\ell$ index individuals.

If a worker ended period $t-1$ unemployed in sector $k$ she can either search in sector $k$ at time $t$ (at no additional cost) or incur a moving cost $C_{k k^{\prime}}$ and search in sector $k^{\prime}$ at time $t$ - so that $C_{k k}=0$. If a worker $\ell$ is not employed at the production stage at $t$, she receives preference shocks $\left\{\omega_{k, \ell}^{t}, k=1, \ldots, K\right\}$ for each sector at time $t$. After unemployed workers receive these shocks, the household head decides whether to keep each worker in the same sector and restrict him to search there at $t$, or to incur a mobility cost and allow him to search in another sector. The $\omega_{k, \ell}^{t}$ shocks 
are iid across individuals, sectors and time, and are assumed to follow a Gumbel distribution with parameters $\left(-\gamma^{E M} \zeta, \zeta\right)$ where $\gamma^{E M}$ is the Euler-Mascheroni constant and $\zeta$ its shape parameter. This structure follows closely Artuç et al. (2010).

After being allocated to search in sector $k^{\prime}$ at $t$ the unemployed worker receives unemployment utility $b_{k^{\prime}}$ and matches with a firm with probability $\theta_{k^{\prime}}^{t} q\left(\theta_{k^{\prime}}^{t}\right)$. We follow Mortensen and Pissarides (1994) and assume that once a worker and a firm match at $t$, a match-specific productivity for $t+1$ production, $x_{\ell}^{t+1}$, is randomly drawn from a distribution $G_{k, i}$ with $[0, \infty)$ support. This productivity is constant over time from then on. At this point, the household head or the firm can break a match if keeping it active is not optimal. Finally, at the end of every period, and following the matching process, there is an exogenous probability $\chi_{k}$ of existing matches to dissolve (excluding new ones). Successful matches that occur at time $t$ only start to produce at $t+1$, and workers employed in sector $k$ are then paid wages denoted by $w_{k}^{t+1}\left(x_{\ell}^{t+1}\right)$. Finally, if a worker produces in sector $k$, she receives a non-pecuniary benefit of $\eta_{k}$. Figure 1 details the timing of the model. Section 2.5 describes the bargaining process that occurs at $t_{a}$ and section 2.4.2 describes the decision of firms to post vacancies at time $t_{c}$.

Figure 1: Timing of the Model

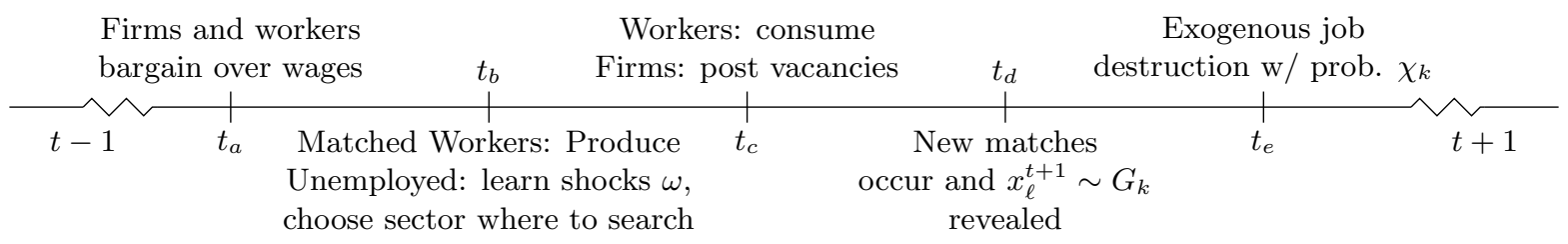

Given this setup, the period utility for individual $\ell$ at time $t$ is,

$$
\mathcal{U}_{\ell}^{t}\left(e_{\ell}^{t}, k_{\ell}^{t+1}, k_{\ell}^{t}, \omega_{\ell}^{t}, c_{\ell}^{t}\right)=\left(1-e_{\ell}^{t}\right)\left(-C_{k_{\ell}^{t}, k_{\ell}^{t+1}}+b_{k_{\ell}^{t+1}}+\omega_{k_{\ell}^{t+1}, \ell}^{t}\right)+e_{\ell}^{t} \eta_{k_{\ell}^{t}}+u\left(c_{\ell}^{t}\right)
$$

where $k_{\ell}^{t}$ is the sector where individual $\ell$ starts period $t$ (that sector was determined at $t-1$ ), $k_{\ell}^{t+1}$ is the $t+1$ sector of choice (which is decided at time $t$, interim period $t_{b}$ in Figure 1 ), $e_{\ell}^{t}$ is the employment status at the production stage (interim period $\left.t_{b}\right), \omega_{\ell}^{t}=\left(\omega_{1, \ell}^{t}, \ldots, \omega_{K, \ell}^{t}\right)$ are idiosyncratic utility shocks received by unemployed workers at interim period $t_{b}$, and $c_{\ell}^{t}$ is individual consumption. If individual $\ell$ is unemployed in sector $k$ at $t\left(e_{\ell}^{t}=0, k_{\ell}^{t}=k\right)$, the individual can switch sectors (from $k$ to $k_{\ell}^{t+1}$ ), so that mobility $\operatorname{costs} C_{k_{\ell}^{t}, k_{\ell}^{t+1}}$, utility of unemployment $b_{k_{\ell}^{t+1}}$ and shock $\omega_{k_{\ell}^{t+1}, \ell}^{t}$ are incurred (during period $t$ ). On the other hand, if individual $\ell$ is employed during the production stage at $t, e_{\ell}^{t}=1$, the worker enjoys the non-pecuniary benefit of working in sector $k_{\ell}^{t}$ given by $\eta_{k_{\ell}^{t}}$. Finally, individual $\ell$ also enjoys the utility of consumption $u\left(c_{\ell}^{t}\right)$.

From the perspective of the household head, the allocation of workers follows a controlled stochastic process: while the head can choose workers' sectors given knowledge of switching costs 
and shocks, employment itself remains a probabilistic outcome. To this end, let $\widetilde{e}_{k}^{t}\left(x_{\ell}^{t+1}\right) \in\{0,1\}$ indicate whether the household head continues on with a match at time $t$ given a match productivity of $x_{\ell}^{t+1}$ in sector $k$. Then, the probability that worker $\ell$ is employed in sector $k$ at time $t+1$, conditional on match productivity $x_{\ell}^{t+1}$ and time $t$ information $\left(k_{\ell}^{t}, e_{\ell}^{t}\right)$ is given by:

$$
\begin{aligned}
\operatorname{Pr}\left(k_{\ell}^{t+1}=k, e_{\ell}^{t+1}=1 \mid x_{\ell}^{t+1}, k_{\ell}^{t}, e_{\ell}^{t}\right) & =\mathcal{I}\left(k_{\ell}^{t}=k\right) e_{\ell}^{t}\left(1-\chi_{k}\right) \widetilde{e}_{k}^{t}\left(x_{\ell}^{t+1}\right) \\
& +\left(1-e_{\ell}^{t}\right) \mathcal{I}\left(k_{\ell}^{t+1}=k\right) \theta_{k}^{t} q\left(\theta_{k}^{t}\right) \widetilde{e}_{k}^{t}\left(x_{\ell}^{t+1}\right) .
\end{aligned}
$$

In words, if $\mathcal{I}\left(k_{\ell}^{t}=k\right) e_{\ell}^{t}=1$, then worker $\ell$ is employed in sector $k$ at time $t$ and the match survives with probability $\left(1-\chi_{k}\right)$ if the family planner decides to keep the match $\left(\widetilde{e}_{k}^{t}\left(x_{\ell}^{t+1}\right)=1\right)$. If $e_{\ell}^{t}=0$, that is, the worker is unemployed at $t$, and the planner chooses $k_{\ell}^{t+1}=k$, then the worker is employed in sector $k$ at time $t+1$ with probability $\theta_{k}^{t} q\left(\theta_{k}^{t}\right) \widetilde{e}_{k}^{t}\left(x_{\ell}^{t+1}\right)$. Importantly, workers' sector and employment status at $t+1, k_{\ell}^{t+1}$ and $e_{\ell}^{t+1}$, are determined by actions taken at $t$.

The head of the household aggregates (4) over family members and maximizes the net present value of utility subject to her budget constraint and the controlled process on employment (5). In addition to consumption and employment decisions, the household head has access to international financial markets by means of buying and selling one-period riskless bonds that are available in zero net supply around the world. One can think of international bond markets in period $t$ as spot markets in which a family buys a piece of paper with face value of $B^{t+1}$ in exchange for a bundle of goods with the same value, and the piece of paper represents a promise to receive goods in period $t+1$ with a value equal to $R^{t+1} B^{t+1}$. International bond markets operate without frictions, so that the nominal returns $R^{t+1}$ are equalized across countries. Finally, the household collects and aggregates profits across all firms, $\Pi^{t}$, but takes this lump-sum payment as given. The household head chooses the path of consumption, $c_{\ell}^{t}$, the path of sectoral choices, $k_{\ell}^{t}$, continuation decisions, $\tilde{e}_{k}^{t}(x)$, and bonds, $B^{t}$, to solve:

$$
\max _{\left\{k_{\ell}^{t}, \widetilde{e}_{k}^{t}(.), B^{t}, c_{\ell}^{t}\right\}} E_{0}\left\{\sum_{t=0}^{\infty}(\delta)^{t} \phi^{t} \int_{0}^{\bar{L}} \mathcal{U}_{\ell}^{t} d \ell\right\},
$$

subject to (5) and the budget constraint:

$$
P^{F, t} \int_{0}^{\bar{L}} c_{\ell}^{t} d \ell+B^{t+1}-\Pi^{t}-R^{t} B^{t}-\int_{0}^{\bar{L}}\left(\sum_{k=1}^{K} \mathcal{I}\left(k_{\ell}^{t}=k\right) e_{\ell}^{t} w_{k}^{t}\left(x_{\ell}^{t}\right)\right) d \ell \leq 0
$$

$E_{0}$ denotes expectations with respect to future idiosyncratic shocks $\omega$. The model has no aggregate uncertainty, so that households and firms have perfect foresight of aggregate variables. $\delta$ is the discount factor, common across all countries, and $\phi^{t}$ is an inter-temporal preference shifter that 
the household head experiences in period $t .^{13}$ These shifters can differ across countries. The budget constraint states the family can buy consumption goods or bonds for next period using profits and wage income, net of interest payments (or collections) on past bonds. Let $\tilde{\lambda}^{t}$ be the Lagrange multiplier on the family's budget constraint (7). ${ }^{14}$ The optimality condition with respect to $c_{\ell}^{t}$ is $u^{\prime}\left(c_{\ell}^{t}\right)=\widetilde{\lambda}^{t} P^{F, t}$, so that individual consumption is equalized across individuals within the household: $c_{\ell}^{t}=c^{t} \forall \ell$. Henceforth, we will refer to $c^{t}$ as per capita consumption. Armed with this observation, Online Appendix A shows that the labor supply decisions solving (6) subject to (7) and (5) can be decentralized and written recursively for unemployed and employed workers. We now turn to this recursive formulation.

Let us return to indexing countries by $i$. Since workers are symmetric up to $x$ and $\eta$ in each country, we stop indexing individual workers. We denote by $\widetilde{U}_{k, i}^{t}\left(\omega^{t}\right)$ the value of unemployment in sector $k$, country $i$ at time $t$ conditional on shocks $\omega^{t}$, and by $W_{k, i}^{t}(x)$ the value of employment conditional on match-specific productivity $x$. If we define $\widehat{\phi}_{i}^{t+1} \equiv \frac{\phi_{i}^{t+1}}{\phi_{i}^{t}}$, the sector decision policy and the continuation rule $\widetilde{e}_{k}^{t}($.$) must solve, conditional on shocks \omega^{t}$ :

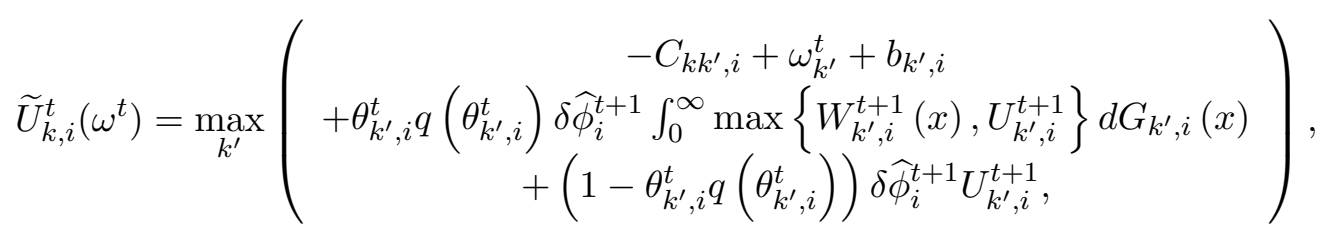

and

$$
W_{k, i}^{t}(x)=\widetilde{\lambda}_{i}^{t} w_{k, i}^{t}(x)+\eta_{k, i}+\delta \widehat{\phi}_{i}^{t+1}\left(1-\chi_{k, i}\right) \max \left\{W_{k, i}^{t+1}(x), U_{k, i}^{t+1}\right\}+\delta \widehat{\phi}_{i}^{t+1} \chi_{k, i} U_{k, i}^{t+1}
$$

In equation (8), $U_{k, i}^{t} \equiv E_{\omega}\left(\widetilde{U}_{k, i}^{t}\left(\omega^{t}\right)\right)$ is the expected value of $\widetilde{U}_{k, i}^{t}\left(\omega^{t}\right)$, integrated over $\omega^{t}$. The first line in this equation corresponds to the costs of switching sectors, $-C_{k k^{\prime}, i}+\omega_{k^{\prime}, i}^{t}$, as well as the sector-specific value of being unemployed in that sector $b_{k^{\prime} i}$. The second line is the probability of finding a match $\theta_{k^{\prime}, i}^{t} q\left(\theta_{k^{\prime}, i}^{t}\right)$ multiplied by the discounted value of the match. Note that for low values of $W_{k^{\prime}, i}^{t+1}(x)$, the household head dissolves the match so that the worker obtains $U_{k^{\prime}, i}^{t+1}$. Finally, the third line is the discounted value of being unemployed next period if the worker fails to successfully match.

In equation $(9), w_{k, i}^{t}(x)$ is the wage paid by a firm with match productivity $x$. Note that it is

\footnotetext{
${ }^{13}$ As will become clear later, inter-temporal preference shocks are going to be important for our model to match the time-series behavior of final expenditures across countries in the data. The use of these shifters is common in the international macroeconomics literature. See Stockman and Tesar (1995) or Bai and Ríos-Rull (2015). However, as discussed in the introduction, the fact that these shifters lead to wedges in Euler equations implies that they can also be viewed as generated by frictions underlying asset markets that directly affect households' aggregate saving decisions.

${ }^{14}$ In an abuse of terminology we will continue to refer to $\tilde{\lambda}^{t}$ as the Lagrange multiplier. However, the correct shadow price associated with period's $t$ budget constraint is given by $\delta^{t} \phi^{t} \widetilde{\lambda}^{t}$.
} 
multiplied by the household head's Lagrange multiplier on the budget constraint $\widetilde{\lambda}_{i}^{t}$. To decentralize the household's problem, individual workers must take into account the effect of their labor supply decisions on the whole family's utility. The second term is the non-pecuniary benefit of working in sector $k$ in country $i$. The next terms are the continuation values: with probability $\left(1-\chi_{k, i}\right)$ the match does not exogenously dissolve and the worker continues the match; with probability $\chi_{k, i}$ the match exogenously breaks and the worker receives the value of unemployment in $k$. Since $\omega$ is Gumbel distributed, the policy rule for unemployed workers can be solved analytically, so that transition rates between $t$ and $t+1$ can be written as:

$$
s_{k k^{\prime}, i}^{t, t+1}=\frac{\exp \left\{\begin{array}{c}
-C_{k k^{\prime}, i}+b_{k^{\prime}, i}+ \\
\left.\theta_{k^{\prime}, i}^{t} q\left(\theta_{k^{\prime}, i}^{t}\right) \delta \widehat{\phi}_{i}^{t+1} \int_{0}^{\infty} \max \left\{W_{k^{\prime}, i}^{t+1}(x)-U_{k^{\prime}, i}^{t+1}, 0\right\} d G_{k^{\prime}, i}(x)+\delta \widehat{\phi}_{i}^{t+1} U_{k^{\prime}, i}^{t+1}\right\}^{1 / \zeta_{i}}
\end{array}\right.}{\sum_{k^{\prime \prime}=1}^{K} \exp \left\{\begin{array}{c}
-C_{k k^{\prime \prime}, i}+b_{k^{\prime \prime}, i}+ \\
\left.\theta_{k^{\prime \prime}, i}^{t} q\left(\theta_{k^{\prime \prime}, i}^{t}\right) \delta \widehat{\phi}_{i}^{t+1} \int_{0}^{\infty} \max \left\{W_{k^{\prime \prime}, i}^{t+1}(x)-U_{k^{\prime \prime}, i}^{t+1}, 0\right\} d G_{k^{\prime \prime}, i}(x)+\delta \widehat{\phi}_{i}^{t+1} U_{k^{\prime \prime}, i}^{t+1}\right\}^{1 / \zeta_{i}}
\end{array}\right.}
$$

\section{$2.4 \quad$ Firms}

\subsubsection{Incumbents}

Firms produce by combining the labor of one single worker with composite intermediate inputs purchased from all sectors. All firms producing variety $j$ in sector $k$ and country $i$ have access to common productivity $z_{k, i}^{t}(j)$ and are paid $p_{k, i}^{t}(j)$ for each unit of production. A firm producing variety $j$ in sector $k$ with match-specific productivity $x$ and employing composite intermediate inputs $\left\{M_{\ell, i}^{t}\right\}_{\ell=1}^{K}$ obtains revenue:

$$
Y_{k, i}^{t}(j, x)=p_{k, i}^{t}(j) z_{k, i}^{t}(j) x^{\gamma_{k, i}}\left(\prod_{\ell=1}^{K}\left(M_{\ell, i}^{t}\right)^{\nu_{k \ell, i}}\right)^{\left(1-\gamma_{k, i}\right)}
$$

where $\gamma_{k, i} \in(0,1), \nu_{k \ell, i}>0$, and $\sum_{\ell=1}^{K} \nu_{k \ell, i}=1$

Firms are price takers in product and intermediate-input markets and decide on the usage of $\left\{M_{\ell, i}^{t}\right\}_{\ell=1}^{K}$ solving:

$$
S_{k, i}^{t}(j, x)=\max _{\left\{M_{\ell, i}^{t}\right\}} p_{k, i}^{t}(j) z_{k, i}^{t}(j) x^{\gamma_{k, i}}\left(\prod_{\ell=1}^{K}\left(M_{\ell, i}^{t}\right)^{\nu_{k \ell, i}}\right)^{\left(1-\gamma_{k, i}\right)}-\sum_{\ell=1}^{K} P_{\ell, i}^{I, t} M_{\ell, i}^{t}
$$

where $S_{k, i}^{t}(j, x)$ denotes the revenue (net of intermediate input payments) generated by the match between a firm and a worker with productivity $x$ producing variety $j$. One can show that:

$$
S_{k, i}^{t}(j, x)=\widetilde{w}_{k, i}^{t}(j) x
$$


where

$$
\begin{gathered}
\widetilde{w}_{k, i}^{t}(j) \equiv \gamma_{k, i}\left(1-\gamma_{k, i}\right)^{\frac{1-\gamma_{k, i}}{\gamma_{k, i}}}\left(P_{k, i}^{M, t}\right)^{\frac{\gamma_{k, i}-1}{\gamma_{k, i}}}\left(p_{k, i}^{t}(j) z_{k, i}^{t}(j)\right)^{\frac{1}{\gamma_{k, i}}} \\
\text { and } P_{k, i}^{M, t} \equiv \prod_{\ell=1}^{K}\left(\frac{P_{\ell, i}^{I, t}}{\nu_{k \ell, i}}\right)^{\nu_{k \ell, i}}
\end{gathered}
$$

is the price of one unit of the intermediate input composite $i m_{k, i}^{t} \equiv \prod_{\ell=1}^{K}\left(M_{\ell, i}^{t}\right)^{\nu_{k \ell, i}}$ in sector $k$ and country $i$. We assume that following entry, switching across varieties within sector is costless for firms. Hence, no arbitrage across varieties will ensure that $\widetilde{w}_{k, i}^{t}(j)=\widetilde{w}_{k, i}^{t}\left(j^{\prime}\right)$ and $p_{k, i}^{t}(j) z_{k, i}^{t}(j)=$ $p_{k, i}^{t}\left(j^{\prime}\right) z_{k, i}^{t}\left(j^{\prime}\right) \forall j, j^{\prime}$. Therefore, $\widetilde{w}_{k, i}^{t}$ and $p_{k, i}^{t} z_{k, i}^{t}$ do not depend on the specific variety. This implies symmetry across varieties, allowing us to drop the index $j$ identifying individual varieties. ${ }^{15}$ Given the expression of the surplus (13), we will henceforth refer to $\left\{\widetilde{w}_{k, i}^{t}\right\}$ as "sectoral surpluses". As will become clearer in Section 2.7 these sectoral surpluses will play the same role as wages do in Caliendo and Parro (2015).

We can write the value function for incumbent firms, $J_{k, i}^{t}(x)$, as:

$$
J_{k, i}^{t}(x)=\widetilde{\lambda}_{i}^{t}\left(\widetilde{w}_{k, i}^{t} x-w_{k, i}^{t}(x)\right)+\left(1-\chi_{k, i}\right) \delta \widehat{\phi}_{i}^{t+1} \max \left\{J_{k, i}^{t+1}(x), 0\right\}
$$

The first term is the firm's current profit, and the second is the firm's continuation value of the match. ${ }^{16}$ If $J_{k, i}^{t}(x)<0$ the firm does not produce and exits.

\subsubsection{New Entrants}

Potential entrants can match with a worker by posting vacancies in $k$ (and variety $j$ ). We assume that posting a vacancy costs $\kappa_{k, i}$ units of the final good, and so amounts to total cost $\kappa_{k, i} P_{i}^{F, t}$. Vacancies are posted at the interim period $t_{c}$ as illustrated in Figure 1 . As argued above, $J_{k, i}^{t}(x)$ is not variety specific, which means the variety distinction can also be ignored for new entrants. If a firm successfully matches with a worker at $t$, production starts at $t+1$. If we denote the expected value of an open vacancy by $V_{k, i}^{t}$, then:

$$
V_{k, i}^{t}=-\widetilde{\lambda}_{i}^{t} \kappa_{k, i} P_{i}^{F, t}+\delta \widehat{\phi}_{i}^{t+1}\left[\begin{array}{c}
q_{i}\left(\theta_{k, i}^{t}\right) \int_{0}^{\infty} \max \left\{J_{k, i}^{t+1}(s), 0\right\} d G_{k, i}(s) \\
+\left(1-q_{i}\left(\theta_{k, i}^{t}\right)\right) \max \left\{V_{k, i}^{t+1}, 0\right\}
\end{array}\right]
$$

\footnotetext{
${ }^{15}$ Remember that we assume that workers face no mobility frictions across varieties within sectors.

${ }^{16}$ Firm profits are multiplied by the multiplier on the family's budget constraint in order to keep the units, utils, consistent between the firm's and worker's problem. However, if one divides $J_{k, i}^{t}(x)$ by $\widetilde{\lambda}_{i}^{t}$, then from the Euler Equation we derive below, it is clear that this formulation is equivalent to a risk neutral firm discounting profits using the nominal interest rate $R^{t+1}$.
} 
The first term on the right hand side is the cost of posting vacancies, which is converted to utility units by the Lagrange multiplier $\widetilde{\lambda}_{i}^{t}$. The second term says that in the next period entrants match with probability $q_{i}\left(\theta_{k, i}^{t}\right)$ and obtain the expected value of $\max \left\{J_{k, i}^{t+1}, 0\right\}$ starting in the next period. If they do not match, they can post another vacancy. To close the model, we impose free entry so that $V_{k, i}^{t} \leq 0 \forall k, i, t{ }^{17}$

\section{$2.5 \quad$ Wages}

The surplus of a match between a worker and a firm is defined as the utility generated by the match in excess of the parties' outside options. Imposing the free entry condition $\left(V_{k, i}^{t}=0\right)$, the outside option to the firm is 0 , while to the worker it is $U_{k, i}^{t}$. Hence, the surplus of the match is given by $\mathcal{S}_{k, i}^{t}(x) \equiv J_{k, i}^{t}(x)+W_{k, i}^{t}(x)-U_{k, i}^{t}$. We assume that firms and workers engage in Nash bargaining over the surplus, with the workers' bargaining weight given by $\beta_{k, i}$. This leads to $J_{k, i}^{t}(x)=\beta_{k, i} \mathcal{S}_{k, i}^{t}(x)$, which combined with equations (9) and (16) imply that $\mathcal{S}_{k, i}^{t}, W_{k, i}^{t}$, and $J_{k, i}^{t}$ are increasing functions of $x$, implying that matches only remain active at $t$ if $x>\underline{x}_{k, i}^{t}$ where $\underline{x}_{k, i}^{t}$ solves:

$$
\mathcal{S}_{k, i}^{t}\left(\underline{x}_{k, i}^{t}\right)=J_{k, i}^{t}\left(\underline{x}_{k, i}^{t}\right)=W_{k, i}^{t}\left(\underline{x}_{k, i}^{t}\right)-U_{k, i}^{t}=0 .
$$

The wage equation resulting from Nash bargaing is:

$$
w_{k, i}^{t}(x)=\beta_{k, i} \widetilde{w}_{k, i}^{t} x+\left(1-\beta_{k, i}\right) \frac{\left(U_{k, i}^{t}-\delta \widehat{\phi}_{i}^{t+1} U_{k, i}^{t+1}-\eta_{k, i}\right)}{\widetilde{\lambda}_{i}^{t}},
$$

for $x \geq \underline{x}_{k, i}^{t}$. This is similar to the standard wage equation in search models: the worker's wage is a weighted average of the surplus, and a function of their outside option. The only new term is the non-pecuniary benefit, which is subtracted from the outside option. By integrating wages across all individuals in the economy, one can solve for the family's total wage income.

\subsection{Labor Market Dynamics}

Since workers can switch sectors between periods $t_{a}$ and $t_{c}$, the sector-specific unemployment rates actually differ at these two points in time within the same period. To this end, we first define the beginning of period sector-specific unemployment rate as $\widetilde{u}_{k, i}^{t-1}$, and labor force as $L_{k, i}^{t-1}$. After workers switch sectors, (measured before matching at $t_{d}$ ), we define $u_{k, i}^{t}$ to be the share of sector- $k$

\footnotetext{
${ }^{17}$ In the equilibria we consider in this paper, we verify that this condition holds with equality, both in steady state and along transition paths.
} 
workers searching for a job. It is given by:

$$
u_{k, i}^{t}=\frac{\sum_{\ell=1}^{K} L_{\ell, i}^{t-1} \widetilde{u}_{\ell, i}^{t-1} s_{\ell k, i}^{t, t+1}}{L_{k, i}^{t}}
$$

where $s_{\ell k, i}^{t, t+1}$ denotes the transition rate from unemployment in sector $\ell$ to search in sector $k$ between $t$ and $t+1$ - see equation (10). $L_{k, i}^{t}$ is the number of workers in sector $k$ at $t$ (more precisely at $t_{c}$ ) and is equal to:

$$
L_{k, i}^{t}=L_{k, i}^{t-1}+\underbrace{\sum_{\ell \neq k} L_{\ell, i}^{t-1} \widetilde{u}_{\ell, i}^{t-1} s_{\ell k, i}^{t, t+1}}_{\text {Inflow }}-\underbrace{L_{k, i}^{t-1} \widetilde{u}_{k, i}^{t-1}\left(1-s_{k k, i}^{t, t+1}\right)}_{\text {Outflow }}
$$

where the second term in the right hand side is the flow of workers into sector $k$ and the third term is the flow of workers out of sector $k$.

Only firms with $x \geq \underline{x}_{k, i}^{t+1}$ produce at $t+1$. Therefore, the number of jobs created in sector $k$ is given by:

$$
J C_{k, i}^{t}=L_{k, i}^{t} u_{k, i}^{t} \theta_{k, i}^{t} q_{i}\left(\theta_{k, i}^{t}\right)\left(1-G_{k, i}\left(\underline{x}_{k, i}^{t+1}\right)\right)
$$

and the number of jobs destroyed is given by:

$$
\begin{aligned}
J D_{k, i}^{t} & \equiv\left(\chi_{k, i}+\left(1-\chi_{k, i}\right) \operatorname{Pr}\left(\underline{x}_{k, i}^{t} \leq x<\underline{x}_{k, i}^{t+1} \mid \underline{x}_{k, i}^{t} \leq x\right)\right) L_{k, i}^{t-1}\left(1-\widetilde{u}_{k, i}^{t-1}\right) \\
& =\left(\chi_{k, i}+\left(1-\chi_{k, i}\right) \max \left\{\frac{G_{k, i}\left(\underline{x}_{k, i}^{t+1}\right)-G_{k, i}\left(\underline{x}_{k, i}^{t}\right)}{1-G_{k, i}\left(\underline{x}_{k, i}^{t}\right)}, 0\right\}\right) L_{k, i}^{t-1}\left(1-\widetilde{u}_{k, i}^{t-1}\right),
\end{aligned}
$$

where $\operatorname{Pr}\left(\underline{x}_{k, i}^{t} \leq x<\underline{x}_{k, i}^{t+1} \mid \underline{x}_{k, i}^{t} \leq x\right)$ is the share of active firms above the productivity threshold at $t$ but below at $t+1$ (endogenous exit). Consequently, the rate of unemployment at the end of period $t$, after job creation and job destruction, is given by:

$$
\widetilde{u}_{k, i}^{t}=\frac{L_{k, i}^{t} u_{k, i}^{t}-J C_{k, i}^{t}+J D_{k, i}^{t}}{L_{k, i}^{t}}
$$

Equations (20)-(24) describe the evolution of labor market stocks over time. In any given period, these stocks are bound by the labor market clearing condition:

$$
\sum_{k=1}^{K} L_{k, i}^{t}=\bar{L}_{i}
$$




\subsection{International Trade}

Our model of international trade closely follows Caliendo and Parro (2015). Varieties are traded across countries. Under the assumptions of perfect competition and iceberg trade costs, the cost of variety $j$ from sector $k$ produced in $o$ can be purchased in country $i$ at a price $p_{k, o}^{t}(j) d_{k, o i}^{t}$, where the first term is the price of variety $j$ in country $o$ and the second term is the iceberg trade cost of shipping from country $o$ to country $i$. From (14) we can write:

$$
p_{k, i}^{t}(j)=\frac{c_{k, i}^{t}}{z_{k, i}^{t}(j)},
$$

for each variety $j$ and where

$$
c_{k, i}^{t} \equiv\left(\frac{\widetilde{w}_{k, i}^{t}}{\gamma_{k, i}}\right)^{\gamma_{k, i}}\left(\frac{P_{k, i}^{M, t}}{1-\gamma_{k, i}}\right)^{1-\gamma_{k, i}}
$$

Recall that due to the no-arbitrage condition we impose across varieties, $c_{k, i}^{t}$ is equalized within sectors, so that price variation across varieties is dictated by $z_{k, i}^{t}(j)$.

We assume that in any country $i$, sector $k$ and period $t$, the productivity component $z_{k, i}^{t}(j)$ is independently drawn from a Frechet distribution with scale parameter $A_{k, i}^{t}$ - which is country, sector, and time specific - and shape parameter, $\lambda$, which is time invariant. ${ }^{18}$ Consumers buy the lowest cost variety across countries, treating the same variety from different origins as perfect substitutes. Under these assumptions, it can be shown that the resulting price index for the composite sector-specific intermediate good (1) is given by:

$$
P_{k, i}^{I, t}=\Gamma_{k, i}\left[\sum_{o=1}^{N} \frac{A_{k, o}^{t}}{\left(c_{k, o}^{t} d_{k, o i}^{t}\right)^{\lambda}}\right]^{-1 / \lambda}
$$

where $\Gamma_{k, i}$ is a constant. In turn, the price of the final good (2) is given by:

$$
P_{i}^{F, t}=\prod_{k=1}^{K}\left(\frac{P_{k, i}^{I, t}}{\mu_{k, i}}\right)^{\mu_{k, i}} .
$$

If we define the object $\Phi_{k, i}^{t} \equiv \sum_{o=1}^{N} \frac{A_{k, o}^{t}}{\left(c_{k, o}^{t} d_{k, o i}^{t}\right)^{\lambda}}$ then consumers in country $i$ spend a share $\pi_{k, o i}^{t}$ of

\footnotetext{
${ }^{18}$ The CDF for the Frechet is given by $F_{k, i}^{t}(z)=\exp \left(-A_{k, i}^{t} \times z^{-\lambda}\right)$.
} 
their sector- $k$ expenditures on varieties from country $o$ given by:

$$
\pi_{k, o i}^{t} \equiv \frac{E_{k, o i}^{t}}{E_{k, i}^{t}}=\frac{A_{k, o}^{t}\left(c_{k, o}^{t} d_{k, o i}^{t}\right)^{-\lambda}}{\Phi_{k, i}^{t}}
$$

where $E_{k, i}^{t}=\sum_{o=1}^{N} E_{k, o i}^{t}$ is the total expenditure of country $i$ on sector $k$ varieties and $E_{k, o i}^{t}$ is the total expenditure of country $i$ on sector $k$ varieties produced by country $o$. Market clearing requires that total revenue $Y_{k, o}^{t}$ coming from the production of varieties in sector $k$ and country $o$ must be equal to sales to all countries $i=1, \ldots, N$, and so:

$$
Y_{k, o}^{t}=\sum_{i=1}^{N} E_{k, o i}^{t}=\sum_{i=1}^{N} \pi_{k, o i}^{t} E_{k, i}^{t}
$$

Let $C_{i}^{t} \equiv \bar{L}_{i} c_{i}^{t}$ denote total consumption of the final good. Define $E_{i t}^{C} \equiv P_{i}^{F, t} C_{i}^{t}$ as total expenditure on final goods, and let $E_{k, i}^{V, t}$ be the total expenditure of sector $k$ in country $i$ on vacancy posting costs. We can write $E_{k, i}^{t}$ as:

$$
E_{k, i}^{t}=\mu_{k, i} E_{i}^{C, t}+\mu_{k, i} \sum_{\ell=1}^{K} E_{\ell, i}^{V, t}+\sum_{\ell=1}^{K}\left(1-\gamma_{\ell, i}\right) \nu_{\ell k, i} Y_{\ell, i}^{t} .
$$

The right hand side represents total expenditure on sector $k$ goods used in final consumption, vacancy posting costs, and as intermediate inputs, respectively. In turn, let $I_{i}^{t}$ denote total disposable income in country $i$, which is given by the portion of revenue that is not devoted to intermediate good payments minus vacancy posting costs, that is, $I_{i}^{t}=\sum_{\ell=1}^{K}\left(\gamma_{\ell, i} Y_{\ell, i}^{t}-E_{\ell, i}^{V, t}\right)$. Net exports are then given by $N X_{i}^{t} \equiv I_{i}^{t}-E_{i}^{C, t}$, and we can rewrite (32) as:

$$
E_{k, i}^{t}=\mu_{k, i}\left(\sum_{\ell=1}^{K} \gamma_{\ell, i} Y_{\ell, i}^{t}-N X_{i}^{t}\right)+\sum_{\ell=1}^{K}\left(1-\gamma_{\ell, i}\right) \nu_{\ell k, i} Y_{\ell, i}^{t}
$$

Finally, labor market clearing dictates that total revenues coming from the production of varieties in sector $k$ and country $i$ is given by:

$$
Y_{k, i}^{t}=\frac{\widetilde{w}_{k, i}^{t}}{\gamma_{k, i}} L_{k, i}^{t-1}\left(1-\widetilde{u}_{k, i}^{t-1}\right) \int_{\underline{x}_{k, i}^{t}}^{\infty} \frac{s}{1-G_{k, i}\left(\underline{x}_{k, i}^{t}\right)} d G_{k, i}(s)
$$

\subsection{Trade Imbalances}

Note that equations (31), (33) and (34) can be solved for any given values of $\left\{N X_{i}^{t}\right\}$, such that $\sum_{i} N X_{i}^{t}=0$. However, these are not necessarily consistent with the household's optimal dynamic 
behavior. To this end, we turn to the determination of net exports $\left\{N X_{i}^{t}\right\}$ in equilibrium. The solution to the household head's problem (6) must satisfy the following Euler equation:

$$
\frac{u^{\prime}\left(c_{i}^{t}\right) / P_{i}^{F, t}}{u^{\prime}\left(c_{i}^{t+1}\right) / P_{i}^{F, t+1}}=\delta \widehat{\phi}_{i}^{t+1} R^{t+1}
$$

and financial and goods markets in each country are linked according to:

$$
N X_{i}^{t}=I_{i}^{t}-P_{i}^{F, t} C_{i}^{t}=B_{i}^{t+1}-R^{t} B_{i}^{t}
$$

Finally, to close this part of the model, we impose that bonds are in zero net supply, $\sum_{i} B_{i}^{t}=0$, and that the initial distribution of bonds is given by $\left\{B_{i}^{0}\right\}$. If the model is initially in steady state, it is easy to verify that $R^{0}=\frac{1}{\delta}$.

Having described the dynamic problem of the household, we can now discuss long-term trade imbalances in our model. To do so, consider beginning from an initial steady state. Suppose that at time $t=1$, the economy unexpectedly experiences a series of shocks that end in finite time, and that a new steady state is reached at time $T .{ }^{19}$ In this case, we arrive at the following equation for the final steady-state value of deficits: ${ }^{20}$

$$
N X_{i}^{T}=-\frac{1-\delta}{\delta}\left(B_{i}^{0} \times \prod_{\tau=1}^{T-1} R^{\tau}+\sum_{t=1}^{T-1}\left(\prod_{\tau=t+1}^{T-1} R^{\tau}\right) N X_{i}^{t}\right)
$$

This equation shows that the behavior of long run imbalances is determined by initial wealth allocations $\left\{B_{i}^{0}\right\}$ and the short-run behavior of net exports $\left\{N X_{i}^{t}\right\}$. This second piece is key in our model: if a country runs a series of trade deficits (surpluses) in the short run, even if they begin with a zero bond position, they may run trade surpluses (deficits) in perpetuity.

\subsection{Equilibrium}

An equilibrium in this model is a set of initial steady-state allocations $\left\{L_{k, i}^{0}, \underline{x}_{k, i}^{0}, B_{i}^{0},\right\}$, a final steady-state allocations $\left\{L_{k, i}^{\infty}, \underline{x}_{k, i}^{\infty}, B_{i}^{\infty},\right\}$ and sequences of policy functions for workers/firms $\left\{s_{k k^{\prime}, i}^{t}, \underline{x}_{k, i}^{t}, w_{k, i}^{t}(x)\right\}$, value functions for workers/firms $\left\{U_{k, i}^{t}, W_{k, i}^{t}, J_{k, i}^{t}\right\}$, labor market tightnesses $\left\{\theta_{k, i}^{t}\right\}$, bond decisions by the households $\left\{B_{i}^{t}\right\}$, bond returns $\left\{R^{t}\right\}$, allocations $\left\{L_{k, i}^{t}, u_{k, i}^{t}, c_{k, i}^{t}\right\}$, profits and household consumption $\left\{\Pi_{i}^{t}, C_{i}^{t}\right\}$, trade shares $\left\{\pi_{k, i o}^{t}\right\}$, sectoral surpluses $\left\{\widetilde{w}_{k, i}^{t}\right\}$, and price indices $\left\{P_{k, i}^{I, t}, P_{k, i}^{F, t}\right\}$ such that:

1. Worker and firms' value functions solve (8), (9), and (16).

\footnotetext{
${ }^{19}$ In our model, because of labor market dynamics, the final steady state is only reached in the limit, but the approximation that the final steady state is reached in finite time is exactly the one used in computation.

${ }^{20}$ We also invoke a transversality condition that $\lim _{T \rightarrow \infty}\left[\prod_{s=1}^{T} R^{s}\right]^{-1} B_{i}^{T} \rightarrow 0 \forall i$.
} 
2. The free entry condition holds in each country and sector: $V_{k, i}^{t}=0 \forall k, i, t$.

3. The wage equation solves the Nash bargaining problem and is given by (19).

4. Allocations and unemployment rates evolve according to (20), (21), (24).

5. Consumption and bonds decisions solve the household's dynamic consumption-savings problem (6) subject to (5) and (7).

6. Price indices are given by equations (28) and (29).

7. Goods markets clear: equations (30)-(32) are met.

8. Labor markets clear: $\sum_{k=1}^{K} L_{k, i}^{t}=\bar{L}_{i}$.

9. Bonds market clears: $\sum_{i=1}^{N} B_{i}^{t}=0$.

10. The initial and final steady-state equilibria satisfy equations (A.15)-(A.36) in Online Appendix B.

\section{Data and Estimation}

Our main source of cross-country data is the World Input Output Database (WIOD), which compiles data from individual National Accounts combined with bilateral international trade data. These data cover 56 sectors and 44 countries, including a Rest of the World aggregate, between 2000 and 2014. We divide the economy into six sectors and six countries. We consider a world comprised by the United States, China, Europe, Asia/Oceania, the Americas and the Rest of the World aggregate constructed by the WIOD. Each country's economic activity consists of six sectors: Agriculture; Low-, Mid- and High-Tech Manufacturing; Low- and High-Tech Services. Tables I and II detail these divisions. We solve our model at the quarterly frequency.

The WIOD dataset allow us to generate various moments that we use in our procedure (detailed below) to estimate a subset of the parameters: (a) employment shares across sectors and countries; (b) average wages across sectors and countries; (c) trade shares; (d) net exports. Other moments used in the estimation procedure are obtained from ILOSTAT and the United States Current Population Survey (CPS). From ILOSTAT, we extract country-specific unemployment rates. From the CPS, we compute yearly transition rates between sectors in the United States, as well as the dispersion of wages. Table III summarizes the statistics targeted in the estimation.

Table IV summarizes the parameters we need to numerically solve the model. We split them into three categories: (i) parameters that are fixed at values previously reported in the literature 
Table I: Country Definitions

\begin{tabular}{ll}
\hline \hline 1 & USA \\
2 & China \\
3 & Europe \\
4 & Asia/Oceania \\
5 & Americas \\
6 & Rest of the World (ROW) \\
\hline \hline
\end{tabular}

Notes: Asia/Oceania $=\{$ Australia, Japan, South

Korea, Taiwan $\}$, Americas $=\{$ Brazil, Canada, Mexico $\}$, Rest of the World $=\{$ Indonesia, India, Russia, Turkey, Rest of the World $\}$

Table II: Sector Definitions

\begin{tabular}{|c|c|c|}
\hline 1 & Agriculture/Mining & Agriculture, Forestry and Fishing; Mining and quarrying \\
\hline \multirow[t]{2}{*}{2} & Low-Tech Manufacturing & Wood products; Paper, printing and publishing; \\
\hline & & $\begin{array}{l}\text { Coke and refined petroleum; Basic and fabricated metals; } \\
\text { Other manufacturing }\end{array}$ \\
\hline \multirow[t]{2}{*}{3} & Mid-Tech Manufacturing & Food, beverage and tobacco; Textiles; \\
\hline & & $\begin{array}{l}\text { Leather and footwear; Rubber and plastics; Non-metallic } \\
\text { mineral products }\end{array}$ \\
\hline \multirow[t]{2}{*}{4} & High-Tech Manufacturing & Chemical products; Machinery; \\
\hline & & cal equipment; Tran \\
\hline \multirow[t]{2}{*}{5} & Low Tech Services & Utilities; Construction; Wholesale and retail trade; \\
\hline & & $\begin{array}{l}\text { Transportation; Accommodation and food service activities; } \\
\text { Activities of households as employers }\end{array}$ \\
\hline 6 & Hi Tech Services & $\begin{array}{l}\text { Publishing; Media; Telecommunications; Financial, real estate } \\
\text { and business services; Government, education, health }\end{array}$ \\
\hline
\end{tabular}

Table III: Summary of Statistics Used in the MSM Procedure

\begin{tabular}{lc}
\hline \hline Statistic & Source \\
\hline Employment allocations across sectors and countries & WIOD \\
Average wages across sectors and countries & WIOD \\
Trade shares across country pairs and sectors & WIOD \\
Net exports across countries & WIOD \\
Unemployment rates across countries & ILOSTAT \\
Coefficient of variation of log-wages in the United States & CPS \\
Yearly transition rates between sectors and from and to & \\
unemployment for the United States & CPS \\
\hline \hline
\end{tabular}


because they are difficult to identify given the available data (Panel A); (ii) parameters that can be determined without having to solve the model (Panel B); and (iii) parameters that are estimated by the method of simulated moments, where we target the moments shown in Table III. ${ }^{21}$

As discussed in Artuç et al. (2010), we are not able to separately identify $\zeta_{i}$ - the dispersion of $\omega$ shocks - and mobility costs $C_{k \ell, i}$ without time variation in wages and transition rates across sectors. For this reason, we follow Artuç et al. (2010) and impose $\zeta_{i}$, to be equal to 1.63 and common across countries. ${ }^{22}$ We then estimate mobility costs following the method of simulated moments procedure we describe below. Given that we solve our model at the quarterly frequency, we impose the discount factor to be $\delta=0.9924$, leading to an annual discount factor of 0.97 - the same value as in Artuç et al. (2010). Flinn (2006) discusses the difficulty in identifying the parameters of matching functions without relying on data on vacancies. We parameterize the matching function according to Coşar et al. (2016): $q_{i}(\theta)=\left(1+\theta^{\xi_{i}}\right)^{1 / \xi_{i}}$ and impose their estimate $\xi_{i}=1.84$ to be common across countries. ${ }^{23}$ Flinn (2006) also highlights the difficulty in estimating the bargaining power parameters without firm-level data. As a result, we follow standard practice in the search literature and set $\beta_{k, i}=0.5$ (Mortensen and Pissarides, 1999). The Frechet scale parameter $\lambda=4$ comes from Simonovska and Waugh (2014). ${ }^{24}$ Finally, we assume individuals have log utility over consumption, $u(c)=\log (c)$. As described in Panel B, the WIOD dataset allows us to directly extract various parameters of the model such as country-specific final expenditure shares $\mu_{k, i}$, labor expenditure shares $\gamma_{k, i}$ and input-output matrices $\nu_{k \ell, i}$; this follows from the Cobb-Douglas assumptions we imposed in the production functions and on how final consumption is aggregated.

We estimate the parameters described in Panel $\mathrm{C}$ using the method of simulated moments. Let $\Theta=\left(\Theta_{1}, \ldots, \Theta_{N}\right)$ be the vector of these country-specific parameters. Our estimation procedure assumes that the economy is in steady state in 2000 and conditions on the observed 2000 trade shares $\pi_{k, o i}^{D a t a}$ and net exports $N X_{i}^{D a t a}$ —so these moments are perfectly matched. Online Appendix B summarizes the equations that must be satisfied in a steady-state equilibrium. For a given guess of $\Theta$, we use our equilibrium equations, conditional on $\pi_{k, o i}^{\text {Data }}$ and $N X_{i}^{\text {Data }}$, to generate: (a) unemployment rates across countries; (b) employment allocations and average wages across sectors and countries; (c) yearly transition rates between sectors and from and to unemployment in the United States; and (d) cross-sectional wage dispersion in the United States. The estimation procedure searches for a vector of parameters $\Theta$ that minimizes the distance between the model

\footnotetext{
${ }^{21}$ To be precise, we do not target trade shares and net exports, we impose them in the estimation, so that they are matched exactly.

${ }^{22}$ Estimates of $\zeta_{i}$ in the literature range from 0.7 in Denmark (Traiberman, 2019) to 2.1 in Brazil (Dix-Carneiro, 2014). The value of 1.63 in Artuç et al. (2010) is well within these bounds, making it a conservative choice. In addition, Traiberman (2019) estimates $\zeta_{i}=1.9$ in Denmark when the method in Artuç et al. (2010) is applied, which disregards worker heterogeneity and human capital accumulation.

${ }^{23}$ One convenient property of this functional form is that it always leads to $q_{i}(\theta)$ and $\theta q_{i}(\theta)$ to be bounded by 0 and 1 .

${ }^{24}$ Caliendo and Parro (2015) find a similar value for $\lambda$ when it is imposed to be equal across sectors: 4.55 .
} 
Table IV: Summary of Parameters

\begin{tabular}{lcll}
\hline \hline & \multicolumn{2}{c}{ Panel A. Fixed According to the Literature } \\
\hline Parameter & Value & Description & Source \\
\hline$\delta$ & 0.9924 & Discount factor & Artuç et al. (2010) \\
$\zeta_{i}$ & 1.63 & Dispersion of $\omega$ shocks & Artuç et al. (2010) \\
$\xi_{i}$ & 1.84 & Matching Function & Coşar et al. (2016) \\
$\beta_{k, i}$ & 0.5 & Worker Bargaining Power & Mortensen and Pissarides (1999) \\
$\lambda$ & 4 & Frechet Scale Parameter & Simonovska and Waugh (2014) \\
\hline \hline \multicolumn{5}{c}{ Panel B. Estimated Outside of the Model } \\
\hline Parameter & Description & Source \\
\hline$\mu_{k, i}$ & Final Expenditure Shares & WIOD \\
$\gamma_{k, i}$ & Labor Expenditure Shares & WIOD \\
$\nu_{k \ell, i}$ & Input-Output Matrix & WIOD \\
\hline \hline \multicolumn{5}{c}{ Panel C. Estimated by Method of Simulated Moments } \\
\hline Parameter & Description \\
$\widetilde{\kappa}_{k, i}$ & Vacancy Costs \\
$\chi_{k, i}$ & Exogenous Exit \\
$\sigma_{k, i}^{2}$ & Distribution of Match-Specific Prod. \\
$\eta_{k, i}$ & Sector-specific Utility \\
$C_{k k^{\prime}}$ & Mobility Costs \\
$b_{k, i}$ & Unemployment Utility \\
\hline \hline
\end{tabular}

Note: Artuç et al. (2010) use an annual discount factor of $\delta=0.97$. Since we work at the quarterly frequency, we use $\delta=0.97^{\frac{1}{4}}$. The distribution of match-specific productivities is imposed to follow a log-normal distribution $G_{k, i} \sim \log \mathcal{N}\left(0, \sigma_{k, i}^{2}\right)$.

generated moments and those we measure in the data.

We impose a few restrictions on the parameters estimated by the simulated method of moments. First, the identification of mobility costs $C_{k \ell, i}$ relies on data on both wages and yearly inter-sectoral transition rates. Although these data can be found in household surveys across a few of the countries we consider, we opted to identify these parameters using transition rates for the United States only. Therefore, we impose mobility costs to be common across countries. Also for identification purposes, we need to impose $C_{k k}=0$ for all $k$. Second, we impose the distribution of match-specific productivities $G_{k, i}$ to follow a log-normal distribution with mean 0 and variance $\sigma_{k, i}^{2}$. We restrict $\sigma_{k, i}^{2}$ to be the same across sectors and countries, and identify it by targeting the cross-sectional coefficient of variation of log-wages in the CPS. Third, we impose that the exogenous exit rates $\chi_{k, i}$ are decomposed into a country component $\chi_{i}$ and a sector component $\chi_{k}$ (that is, $\chi_{k, i}=\chi_{i}+\chi_{k}$ ). The sector-specific component $\chi_{k}$ is identified from US transitions from sector-specific employment to unemployment. The country-specific components are then adjusted to better match the countryspecific unemployment rates. Fourth, the utility of unemployment $b_{k, i}$ is imposed to be country 
specific (that is $b_{k, i}=b_{i}$ ). This parameter will be important for the model to be able to match levels of wages across countries. Finally, sector- and country-specific utility terms $\eta_{k, i}$ help the model simultaneously fit sector-specific wages and employment shares. To achieve identification, we need to impose $\eta_{k_{0}, i}=0$ for some sector $k_{0}$ (we picked $k_{0}=$ Agriculture).

A convenient aspect of our approach is that, by conditioning on the observed 2000 trade shares and trade imbalances, and normalizing total world revenues $\sum_{k} \sum_{i} Y_{k, i}=1$, we can solve for sector-country revenues $\left\{Y_{k, i}\right\}$ that are independent of $\Theta$. Specifically, equations (31), (33), and the normalization lead to a system of equations in $\left\{Y_{k, i}\right\}$, which can be solved before starting the estimation procedure. Consequently, the sector- and country-specific labor demand side of the model is fixed throughout the estimation procedure, allowing the labor supply side in each country to be solved in isolation. To see this, notice that equation (34) contains revenues on the left hand side, and the right hand side only depends on country-specific sectoral variables and parameters. Therefore, in steady state, observed trade flows and trade imbalances are sufficient statistics for international linkages. This property allows us to estimate the model country by country, greatly simplifying the estimation procedure. ${ }^{25}$ Indeed, if all parameters were country specific and we had the same set of data for each country, estimation could be conducted in parallel for each country. As a final point, a convenient aspect of conducting the estimation conditional on the observed trade shares is that we do not have to estimate the technology parameters $A_{k, i}$ and trade costs $d_{k, o i}$. We develop algorithms to perform counterfactual responses to shocks to technology parameters and trade costs relying on the exact hat algebra approach in Dekle et al. (2007), Dekle et al. (2008) and Caliendo and Parro (2015).

Although the model could be flexibly estimated country by country if we had data on yearly transitions across sectors for all countries, we impose $C_{k \ell, i}$ and $\sigma_{k, i}^{2}$ to be constant across countries and identified off the United States' CPS transition rates and wage dispersion data. Therefore, we proceed in two steps. First, we estimate the model for the United States, back out $C_{k \ell}, \sigma_{k}^{2}$ and $\chi_{k}$. In a second step, we estimate the rest of the parameters in parallel, country by country, conditional on the values of $C_{k \ell}, \sigma_{k}$, and $\chi_{k}$ that are estimated in the first step.

At this point, it is worthwhile mentioning that our estimation approach, which does not estimate $A_{k, i}$ and trade costs $d_{k, o i}$, cannot recover $\kappa_{k, i}$. Instead, we are able to recover the value of $\widetilde{\kappa}_{k, i} \equiv$ $\frac{\kappa_{k, i} P_{i}^{F}}{\widetilde{w}_{k, i}}$ in the 2000 steady-state equilibrium. Our counterfactual algorithms then allow $\widetilde{\kappa}_{k, i}$ to respond to shocks, using exact hat algebra. It is also important to clarify that identifying $\widetilde{\kappa}_{k, i}$ is difficult in practice. Even though the objective function is not flat with respect to $\widetilde{\kappa}_{k, i}$, it tends to be relatively flat for a wide range of values. For that reason, we follow Hagedorn and Manovskii (2008) and impose that the cost of posting one vacancy $\kappa_{k, i} P_{i}^{F}$ equals $0.58 \times \bar{w}_{k, i}$, where $\bar{w}_{k, i}$ is the

\footnotetext{
${ }^{25}$ The method of simulated moments objective function is highly non-linear and non-convex, so that global optimization routines, such as Simulated Annealing, must be applied. Breaking a large parameter vector into smaller subsets of parameters that can be estimated separately greatly simplifies the estimation procedure.
} 
average wage in sector $k$ and in country $i$. Hagedorn and Manovskii (2008) base their calibration on a combination of the labor and capital costs of posting vacancies in a single-sector search model. ${ }^{26}$ While their model and ours differ on several dimensions - e.g., we have multiple sectors and match heterogeneity - there are two reasons we turn to their calculation. First, Hagedorn and Manovskii combine micro and macro data from multiple sources, mapping their numbers to observable costs of recruitment, capital purchasing, and other costs; second, their calibration of vacancy posting costs does not rely on other parameters of the model, just observable data (e.g., the capital share of income and the unemployment rate), so that their estimate is not contaminated by the fact that our other parameters are not always estimated to be the same as Hagedorn and Manovskii's. We impose this constraint by adding the deviations between $\left(\widetilde{\kappa}_{k, i} \times \widetilde{w}_{k, i}\right) / \bar{w}_{k, i}=\left(\kappa_{k, i} \times P_{i}^{F}\right) / \bar{w}_{k, i}$ and 0.58 in our method of simulated moments objective function. The full estimation algorithm is described in Online Appendix C.1.

\section{Estimation Results and Mechanisms}

\subsection{Estimates and Model Fit}

The estimated parameters can be found in the Online Appendix C.7, in Tables A.1 through A.8. We first discuss the parameters that are obtained outside of the model. The Online Data Appendix details how these are computed with data from the WIOD. Table A.1 displays the final expenditure shares $\mu_{k, i}$. We can separate the countries in this table in two groups with similar expenditure shares: (1) United States, Europe, Asia, and Americas; and (2) China and Rest of the World. The biggest difference between these two countries is that China and the Rest of the World spend a much larger share of their disposable income on Agricultural goods and significantly lower share on High-Tech Services.

Table A.2 displays the share of revenues devoted to labor payments. This share varies mostly within countries and across sectors and ranges between 0.24 (in High-Tech Manufacturing in China) and 0.68 (in High-Tech Services in Rest of the World). Finally, Table A.3 displays the average across countries of the input-output matrices. As is well known, the diagonal elements tend to be larger than the off-diagonal elements.

Our mobility costs are displayed in Table A.4. Artuç et al. (2010) express their mobility costs estimates as multiples of average wages. In contrast, in our model, workers compute their value functions and make decisions based on $\widetilde{\lambda}_{i} \times \widetilde{w}_{k, i}$, and not just based on sector-specific average wages. Therefore, to make our estimates comparable to those in Artuç et al. (2010), we express

\footnotetext{
${ }^{26}$ As the authors point out, in the steady state of a single sector search model, capital is equivalent to interpreting vacancies as capital and reinterpreting the productivity process. In this sense, a vacancy is a metaphor for the whole cost of hiring a worker - the flow cost of capital, HR efforts, etc.. We adopt a similar view in our paper, without modeling each piece.
} 
(a) Employment Shares

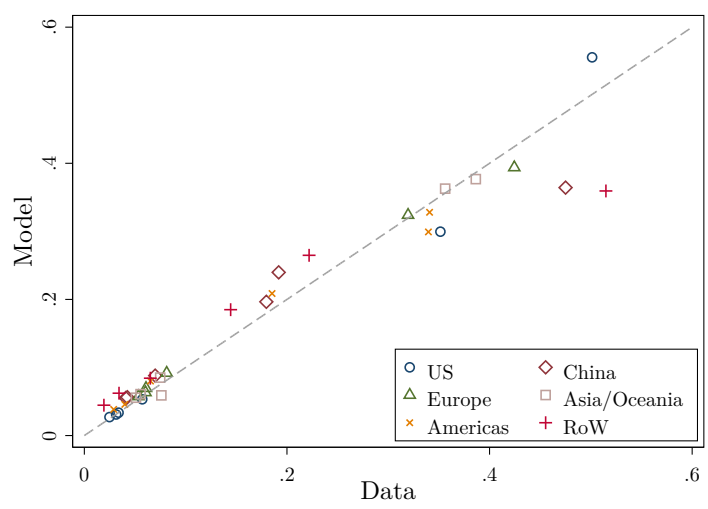

(c) Average Wages

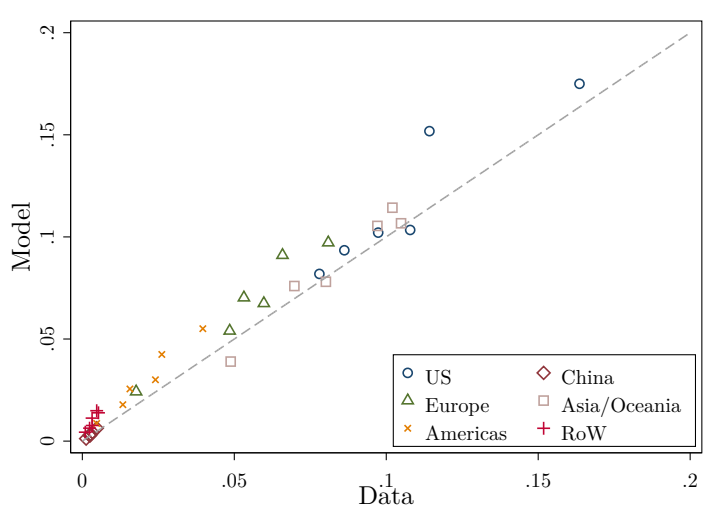

(b) Unemployment Rates

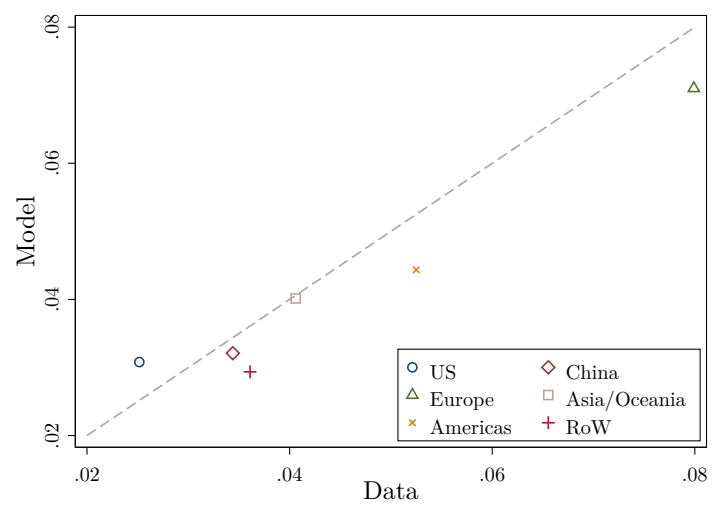

(d) Transition Rates

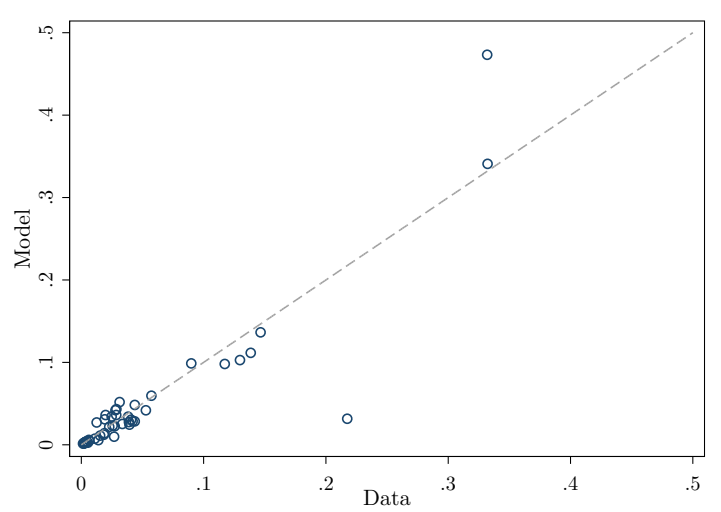

mobility costs as multiples of $\widetilde{\lambda}_{U S} \times \bar{w}_{U S}$, where $\bar{w}_{U S}$ is the average wage in the US (in the data). We obtain that our estimated mobility costs across sectors (as a fraction of $\tilde{\lambda}_{U S} \times \bar{w}_{U S}$ ) range from virtually zero to a maximum of 3.1. These numbers are of the same order of magnitude, but lower than what Artuç et al. (2010) estimate (in their preferred specification, mobility costs are 6 times average wages). We believe that two ingredients of our model absent from theirs, search frictions and sector-specific utility terms $\eta_{k, i}$, account for our lower estimates of mobility costs. Indeed, Artuç and McLaren (2015) extends ACM to allow for $\eta$ terms and also find lower moving frictions. Finally, Table A.7 shows that the values of unemployment $b_{i}$ tend to be negative across all countries. As the search literature has shown, a negative value of unemployment is necessary to generate the magnitudes of wage dispersion typically found in the data (e.g., Hornstein et al., 2011; Meghir et al., 2015).

Figure 2 shows the model fit for the various moments we target: (a) employment shares across sectors and countries; (b) unemployment rates across sectors and countries; (c) average wages across sectors and countries; and (d) inter-sectoral transition rates in the United States. As a general rule, 
the model matches the targeted moments well.

\subsection{Impulse Response Functions}

Endogenizing trade imbalances as optimal inter-temporal consumption decisions implies that the dynamics of the shocks hitting the economy are key for the evolution of imbalances. Hence, in order to understand the rich mechanisms at play in our model, we study the model's behavior in response to three sets of shocks. First, we subject the model to a tenfold increase in $A_{k, C h i n a}$, the productivity location parameter in China, uniform across sectors $k$ and lasting for five years before turning back to its original level - the magnitude of this shock is in line with the size of actual changes in Chinese productivity that we recover in section 5.1. Next, we feed the model with a tenfold permanent increase in $A_{k, \text { China }}$ that happens once and for all at $t=1$. Finally, we simulate a slow-moving and linear increase in $A_{k, \text { China }}$ that reaches a tenfold increase in 15 years. It remains at that level from there on. These shocks are illustrated in Figure 3. The economy is initially in steady state, so that the shocks are unanticipated at $t=0$, but their paths are fully anticipated at $t=1$. To highlight the quantitative and qualitative importance of modeling trade imbalances, we study the behavior of the complete model with bonds as well as the behavior of a model without bonds, where trade is balanced in every period - that is $N X_{i}^{t}=0 \forall i, t{ }^{27}$

We start with the temporary shock depicted in Figure 3a. The evolution of net exports in Figure 4 aligns with what we would have expected from standard macroeconomic models with inter-temporal decisions. The temporary boost in productivity, and therefore income, induces China to save in the short run by running a large trade surplus. As productivity reverts back to its initial level, China sustains a permanent trade deficit of $21 \%$ of GDP, as it consumes the return on its savings. On the other hand, the remaining countries borrow from China, running trade deficits in the short run. As the productivity increase in China vanishes, all countries start to sustain permanent trade surpluses, repaying their debts to China.

While these aggregate patterns are standard, this productivity shock has varied impacts on the more disaggregated economy. Specifically, the rise in productivity induces substantial labor reallocation across sectors. We summarize reallocation patterns relying on the following measure:

$$
\text { Reallocation }_{i}^{t}=\frac{1}{2} \sum_{s=1}^{t} \sum_{k=1}^{J}\left|\frac{L_{i, k}^{s}}{L_{i}^{s}}-\frac{L_{i, k}^{s-1}}{L_{i}^{s-1}}\right|
$$

which accumulates yearly changes in sectoral employment shares over time.

Figure 5a plots the evolution of the reallocation index (38) across countries. When we consider

\footnotetext{
${ }^{27}$ To make these two cases comparable, we study the complete model responses (with trade imbalances) imposing that trade is balanced in the initial steady state. See Online Appendix C.3 for details on how we implement this procedure using exact hat algebra techniques.
} 
Figure 3: Shocks to Chinese Productivity $\widehat{A}_{k, \text { China }}-$ Uniform Across Sectors

(a) Temporary

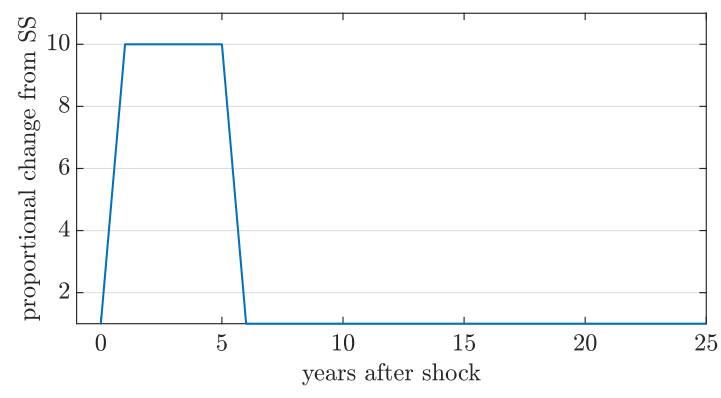

(c) Slow Moving

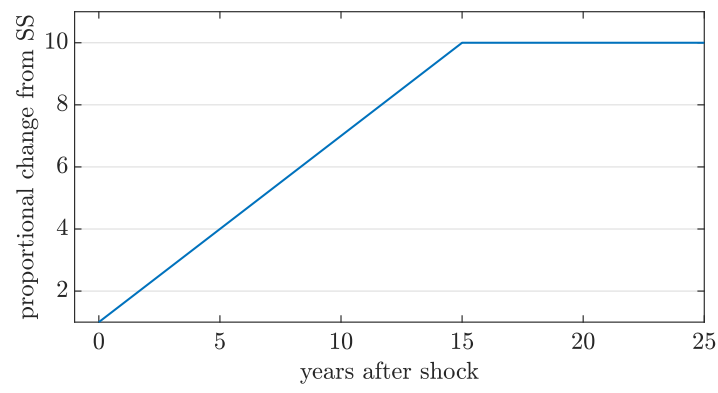

(b) Once-And-For-All

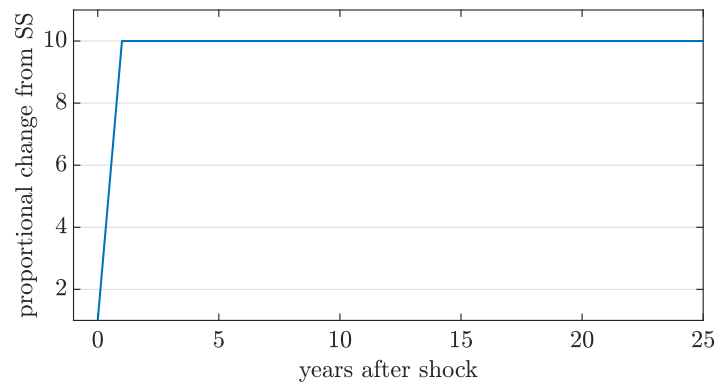

our complete model with bonds, there is an immediate and steep rise in reallocation in all countries following China's productivity jump. After productivity returns to its initial level, reallocation continues for several years before reaching its final steady-state level. Even though we consider a uniform productivity shock across sectors, we observe substantial labor reallocation within China and within other countries. ${ }^{28}$ The reason that uniform productivity changes can have large impacts on labor reallocation is trade across countries, which adds asymmetries to how sectors respond to the same productivity shock. Our model features two sources of asymmetries. First, heterogeneity in trade costs implies that the tradability of goods differs across countries and sectors. As explained by Dornbusch et al. (1977), transfers (here, net exports) across countries alter the terms of trade and therefore the allocation of economic activity within each country. Second, countries differ in their final consumption bundles which implies that the global distribution of final consumption expenditure matters for the allocation of workers across sectors. Relatedly, heterogeneity in inputoutput linkages across countries also generate changes in countries' terms of trade and global expenditure patterns.

The increase in reallocation is directly tied to an increase in unemployment in all countries,

\footnotetext{
${ }^{28}$ Note that in a closed economy without mobility costs and search frictions, we would have seen no reallocation effects given homothetic preferences and our production structure as relative prices would not change. However, the presence of heterogeneous mobility costs across sectors could lead to some reallocation effects as the option value of search would change differentially across sectors. However, based on Shimer (2005) we expect this effect to be quantitatively small.
} 
Figure 4: Net Exports Over GDP in Response to Temporary Productivity Growth in China (Figure 3a)
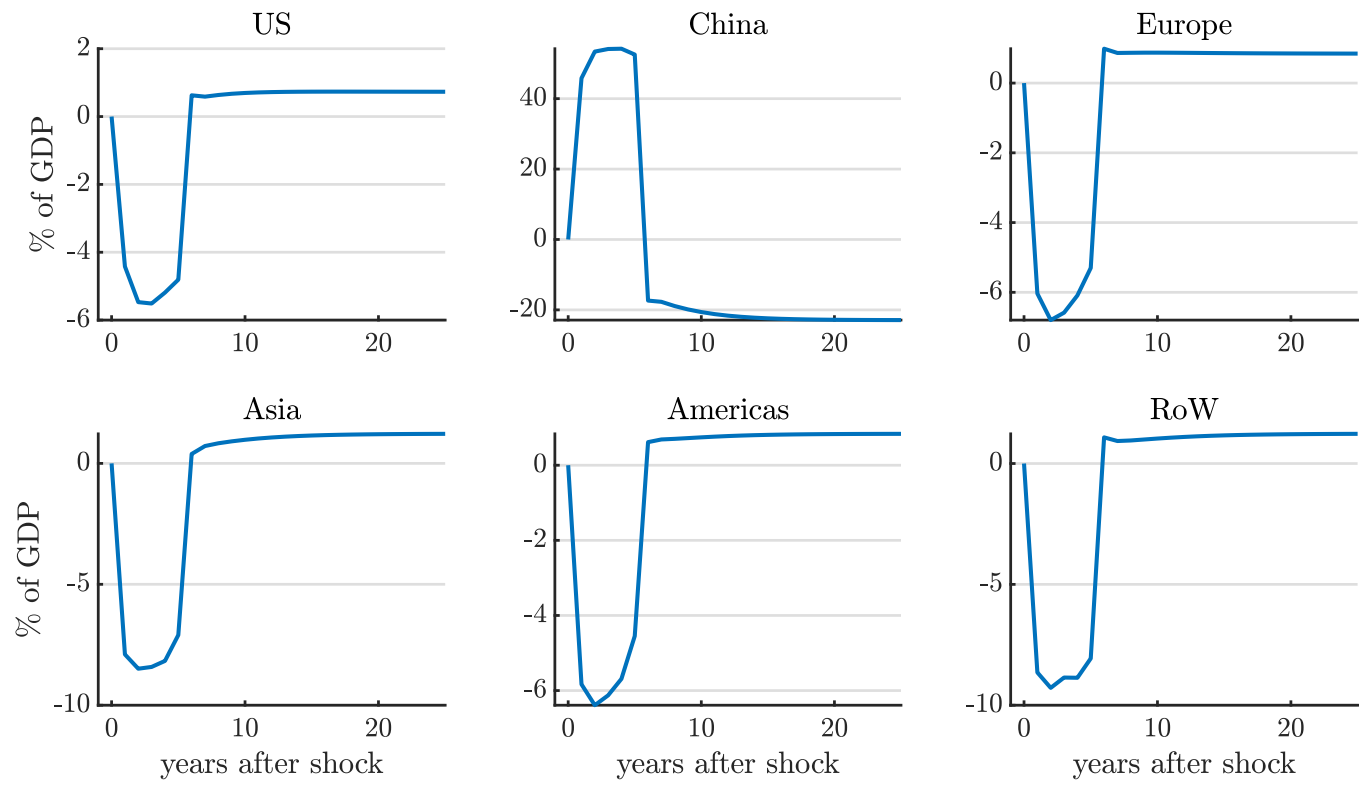

as shown in Figure 5b. This highlights a key mechanism in our model: even positive labor demand shocks can increase unemployment through asymmetric shifts in labor demand. Once again, there are two reasons this can occur. First, contracting sectors will destroy low productivity jobs, dislocating workers who must switch sectors. Second, it takes time for workers who switch into expanding sectors to find new jobs. Relatedly, that there is no systematic relationship between the response of unemployment and the sign of trade imbalances.

The reason why modeling imbalances generates more reallocation is due to the richer patterns of reallocation that emerge, shown in Figures $5 \mathrm{c}$ (for the full model) and $5 \mathrm{~d}$ (for balanced trade). When we consider the complete model with bonds, rising imports in the US following Chinese productivity growth do not need to be immediately met with rising exports. Instead, the US imports goods in the short run and reallocates labor to service sectors, which are less tradable. This pattern follows from homothetic preferences, and so demand for services increases when total consumption in the US rises - as this demand cannot be easily met with imports. However, if we impose balanced trade in every period, exports from the US must immediately rise to offset the increase in imports. In doing so, labor reallocates to the sector in which it has highest comparative advantage (high relative productivity coupled with lower trade costs), which is also the sector in which China has higher relative demand: Agriculture. Reallocation when trade is balanced is driven by trade costs and comparative advantage, which do not change much in response to the temporary productivity growth in China.

Turning to the long run, savings and borrowing behavior leads to permanent changes in final 
Figure 5: Labor Market Dynamics in Response to Temporary Productivity Growth in China (3a)

(a) Reallocation Index
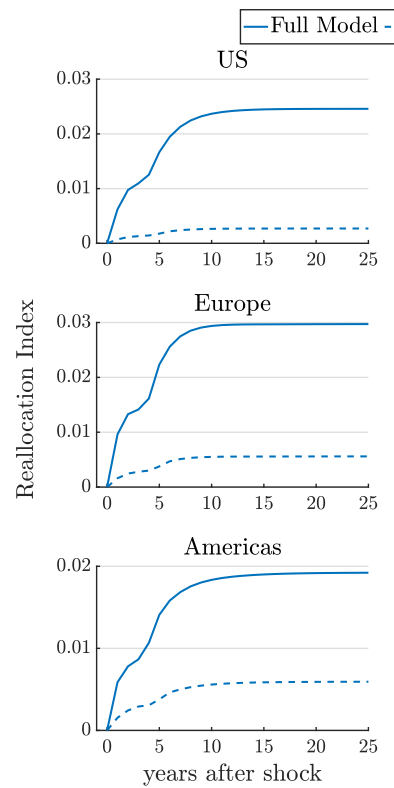

(c) Labor Allocations - Full Model
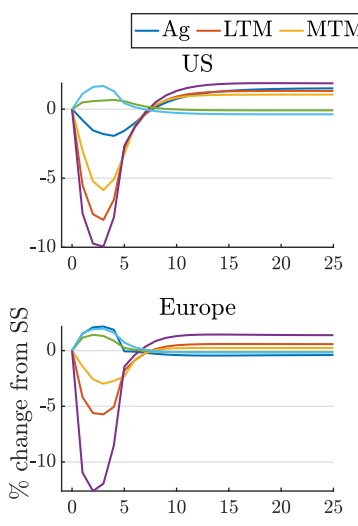

Americas

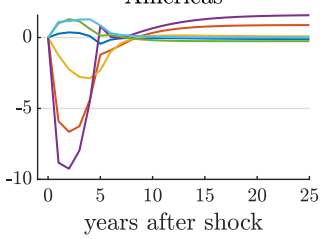

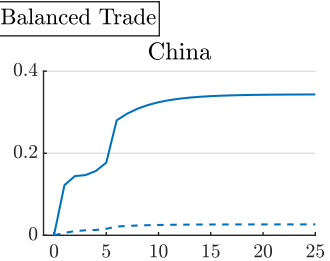
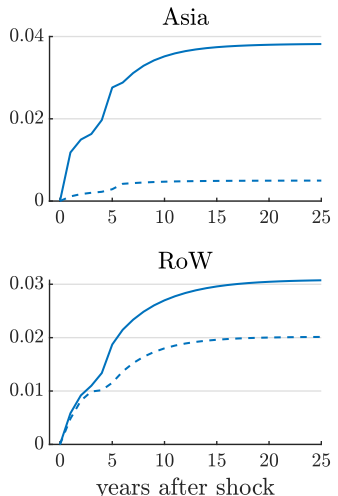

-HTM - LTS - HTS

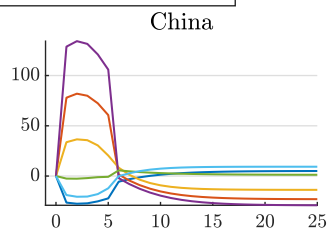

Asia

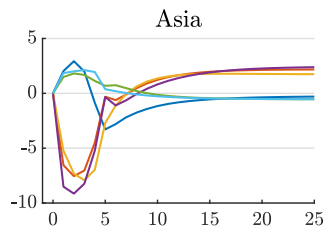

RoW

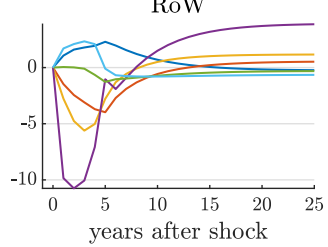

(b) Unemployment

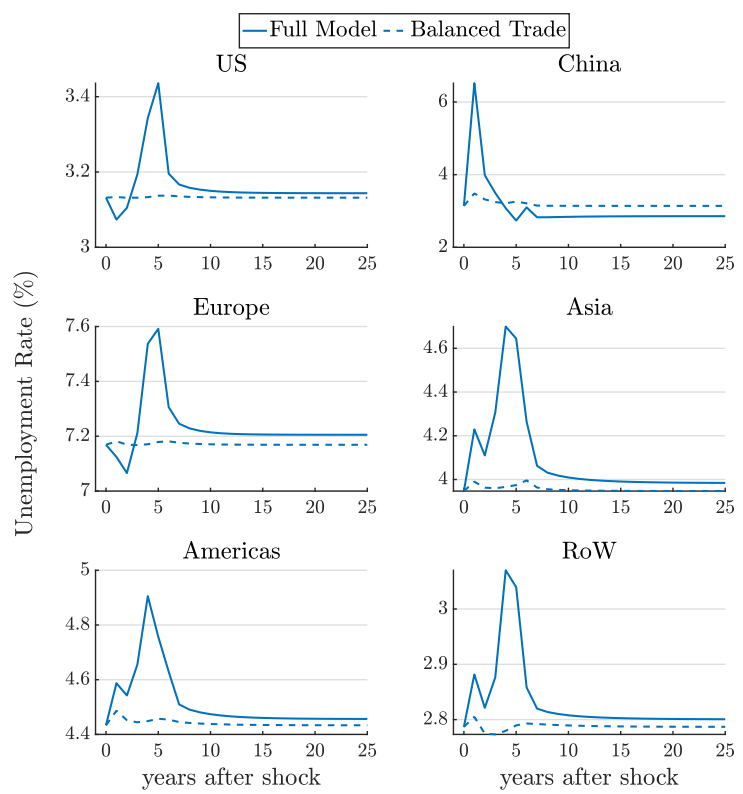

(d) Labor Allocations - Balanced Trade
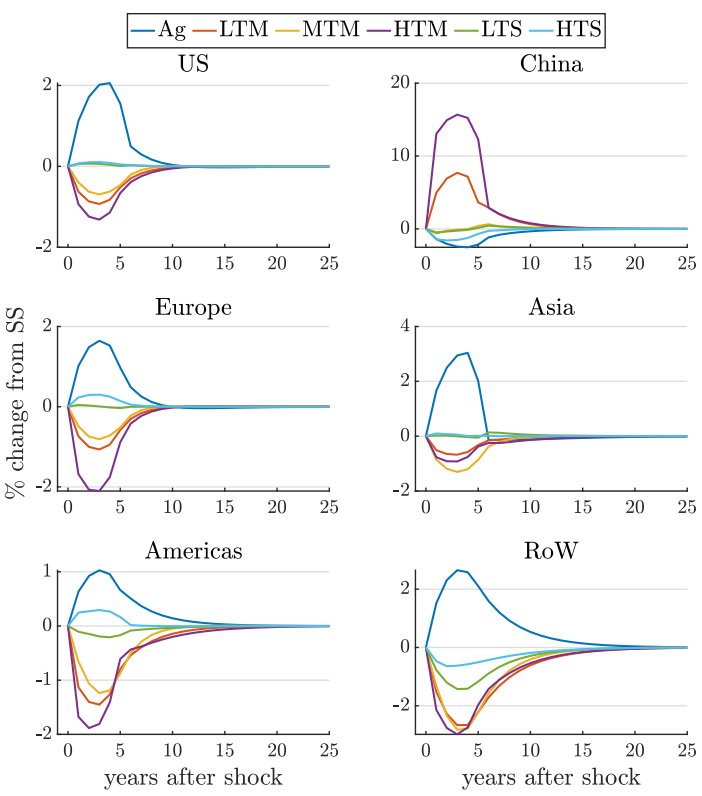

RoW

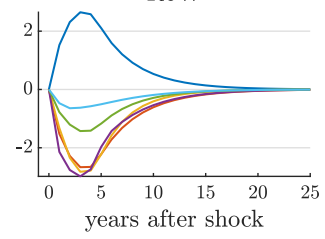


steady-state bond positions across countries. These permanent shifts in the long-run allocation of wealth carry implications for steady-state labor allocations and unemployment. The sources of long-run reallocation are similar to those operating in the short run, except that the effects of the temporary shock on the distribution of bonds positions (and therefore wealth) across countries is permanent. Specifically, the US runs a large deficit in the short run, which must be paid off in the form of long-run trade surpluses. To repay its debt with China, it shifts labor back to its goods sectors which can easily ship their production internationally. In the long run, the goods sectors in the US expand relative to the initial steady state, whereas its service sectors slightly contract. These long-run effects in response to a temporary shock are in sharp contrast with those in the model with balanced trade, which predicts that long-run allocations are unchanged relative to initial ones.

We next turn to the analysis of the once-and-for-all shock in Chinese productivity depicted in Figure 3b. Figure 6 highlights that the effect of this shock on net exports across countries is of much smaller magnitude. Indeed, when an economy is subject to a permanent shock the importance of savings to smooth consumption is of second order. If labor were perfectly mobile across sectors, economies would instantaneously reallocate labor and achieve their unconstrained optimal levels of expenditure. Because labor is not perfectly mobile, we do observe imbalances arising, but these are modest. It is therefore not surprising that, in the event of once-and-for-all permanent shocks, the behavior of the model with imbalances is similar to one without imbalances. ${ }^{29}$

\footnotetext{
${ }^{29}$ However, it is worth pointing out that this prediction of the model could be different for a shock that is not uniform across sectors, or for permanent shocks to trade costs (Reyes-Heroles, 2016; Alessandria and Choi, 2019).
} 
Figure 6: Net Exports Over GDP in Response to Once-and-for-all Productivity Growth in China (Figure 3b)
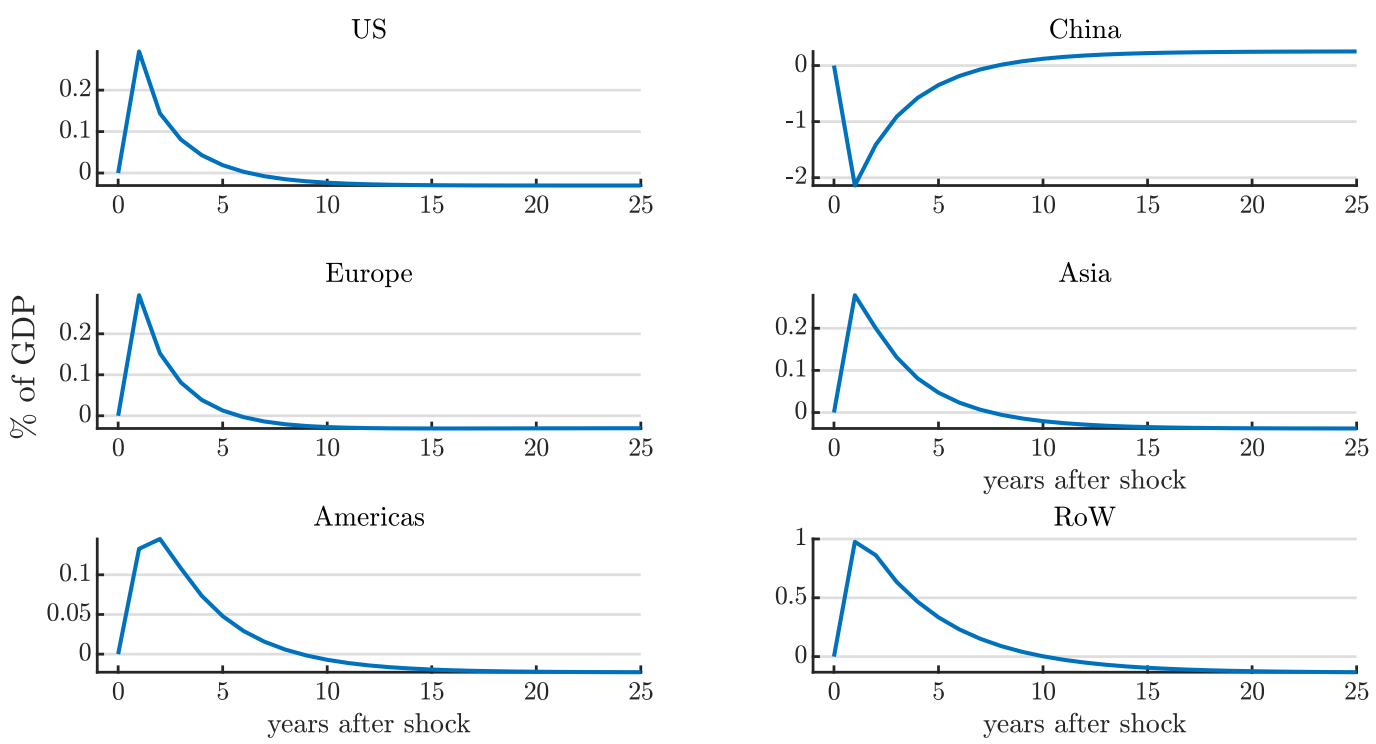

Finally, we study the behavior of our model when we feed it with a slow increase in Chinese productivity, as is illustrated in Figure 3c. Figure 7 shows that, in this case, China heavily borrows in the short run in anticipation of its large increase in long-run productivity. All other countries lend to China by running large surpluses for over ten years. As with the temporary shock, trade imbalances greatly magnify the extent to which reallocation and unemployment respond relative to the trade balance benchmark. Figures $8 \mathrm{a}$ and $8 \mathrm{~b}$ show that the short-run labor market dynamics are very different across the two models. With trade imbalances, Figure $8 \mathrm{c}$ shows that the US reallocates resources away from its service sectors and towards its goods sectors so that their output can be shipped to China. However, in the long run, China needs to repay its debt and so ships goods back to the US. This leads to a long-run contraction of manufacturing in the US and an expansion of the remaining sectors. Compare this behavior with the monotonic decline in manufacturing in the US if trade is imposed to be balanced in Figure 8d. The magnitude of the manufacturing contraction is also much more limited under trade balance.

The main conclusion of this section is that trade imbalances can significantly amplify the reallocation and unemployment responses to temporary shocks. They can also lead to very different patterns of inter-sectoral reallocation in the short and long run. However, the quantitative relevance of endogenizing imbalances depends on the nature of the shock, as illustrated by our once-and-for-all permanent shock example. This fact can be appreciated by comparing the results for the temporary and slow moving shocks. An additional important result worth pointing out from our previous analysis is that the magnitude of reallocation and unemployment responses is independent of the 
sign of the trade imbalance. Finally, our simulations highlight that, with trade imbalances, the full path of shocks matter for the behavior of imbalances over time, but also for the determinantion of their long-run consequences. Given the importance of the path of shocks for trade imbalances and the adjustment process, we now turn to the analysis of the labor market consequences of the shocks the global economy actually experienced between 2000 and 2014.

Figure 7: Net Exports Over GDP in Response to Slow Productivity Growth in China (Figure 3c)
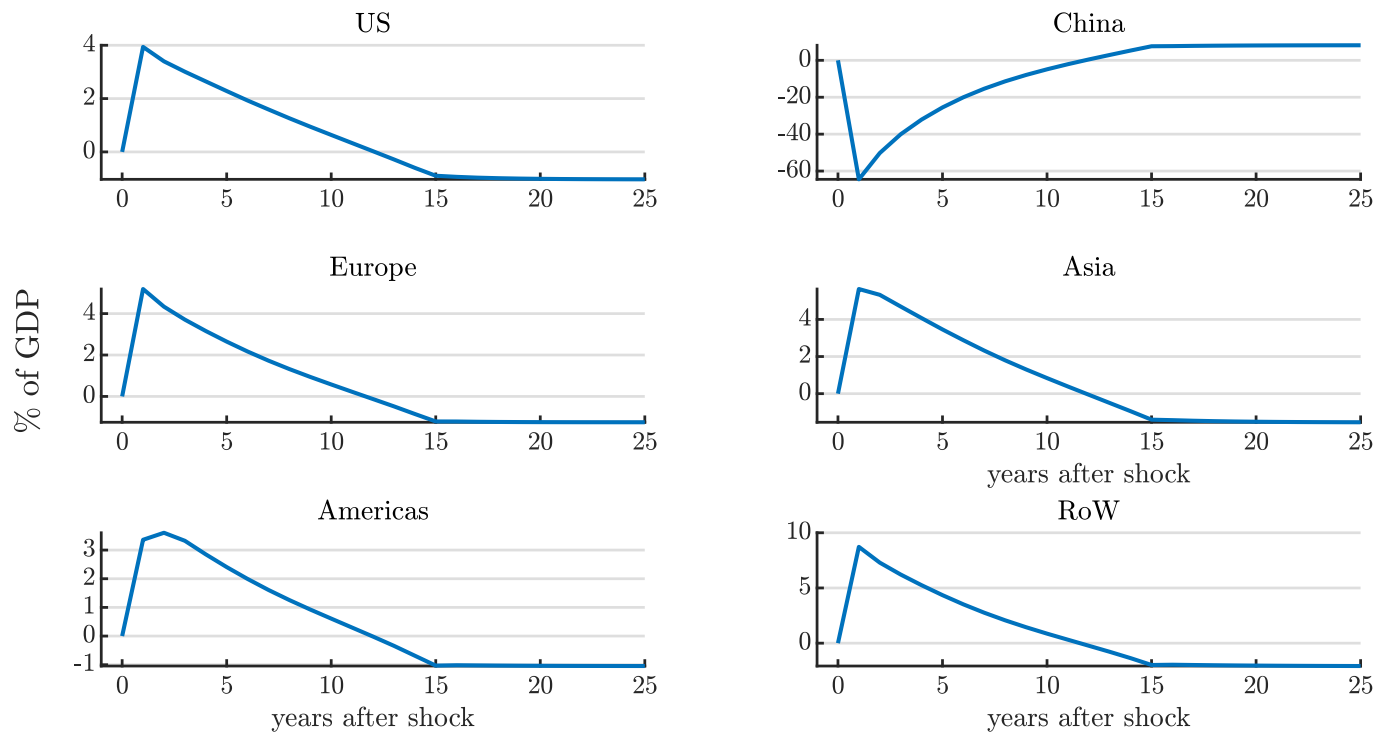

\section{Counterfactuals}

Section 4.2 showed that the exact path of shocks shape the magnitude and evolution of trade imbalances over time, directly influencing long-run outcomes through changes in the long-run global distribution of bond holdings. For this reason, we conduct an empirical exercise in which we extract the various shocks the global economy has actually experienced between 2000 and 2014. Armed with these shocks, we conduct a common exercise in the international trade literature: how do labor markets behave over time in response to the constellation of globalization shocks (i.e., shocks to productivity and trade costs)? In this exercise, we compare the labor markets responses that we obtain with our model of trade imbalances to the responses that we obtain imposing balanced trade. Having established the quantitative importance of trade imbalances for both the adjustment process and long-run allocations, we compare the consumption gains in response to changes in trade costs in our model to those obtained in standard models of trade, as summarized by the sufficient statistic approach developped by Arkolakis et al. (2012). Next, we compute the globalization gains accrued to each country between 2000 and 2014, and compare them to those obtained in a world 
Figure 8: Labor Market Dynamics in Response to Slow Productivity Growth in China (Figure 3c)

(a) Reallocation Index
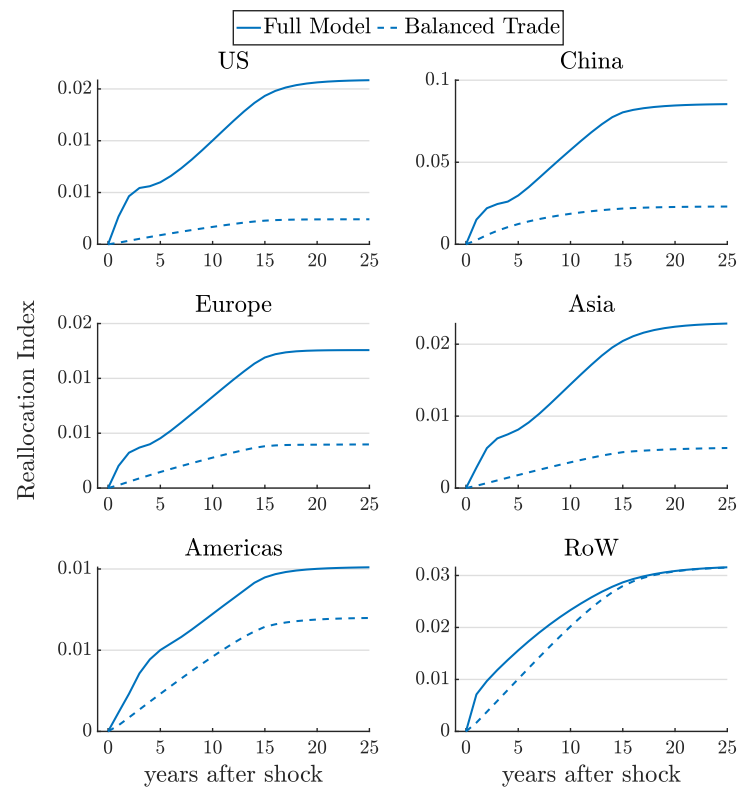

(c) Labor Allocations - Full Model
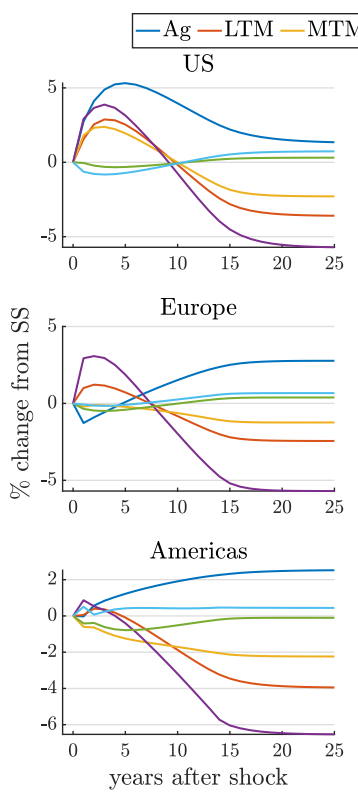
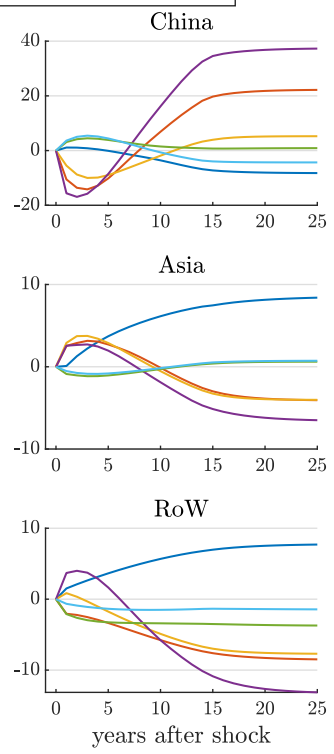

(b) Unemployment

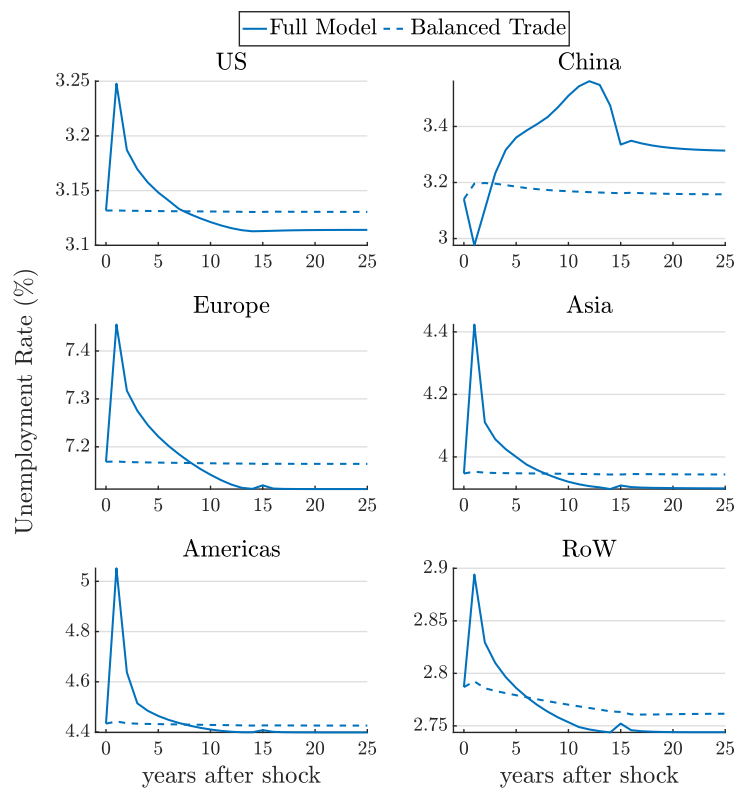

(d) Labor Allocations - Balanced Trade
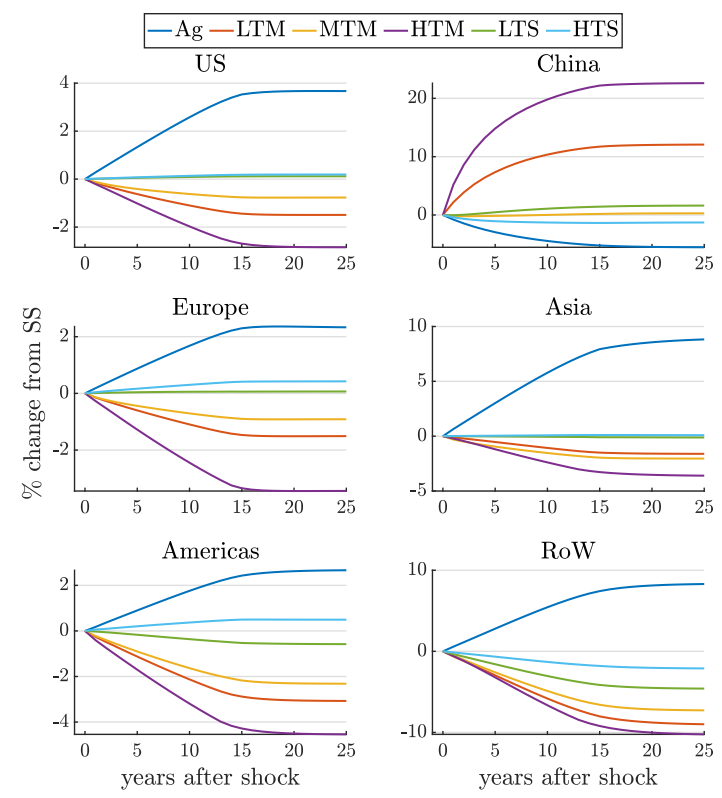
without the global savings glut, or in a world with balanced trade (i.e., no bonds). Finally, given the recent interest on the "China shock" on the US labor market, we use our extracted shocks to study the effects of shocks to the Chinese economy on the behavior of unemployment and manufacturing employment in the US. We also investigate the contribution of the "China shock" for the US trade deficit and for the trade surplus in China.

\subsection{Extracting Shocks from the Data}

This section obtains the time series for three sets of shocks in the global economy between December of 2000 and December of 2014: changes in trade costs $\left\{\widehat{d}_{k, o i}^{t}\right\}$, inter-temporal preference shocks $\left\{\widehat{\phi}_{i}^{t}\right\}$ and productivity shocks $\left\{\widehat{A}_{k, i}^{t}\right\}$. We measure changes in trade costs and productivity relative to December of 2000 (which we label $t=0$ ): $\widehat{d}_{k, o i}^{t}=\frac{d_{k, o i}^{t}}{d_{k, o i}^{0}}, \widehat{A}_{k, i}^{t}=\frac{A_{k, i}^{t}}{A_{k, i}^{0}}$. On the other hand, shocks to inter-temporal preferences are relative to the previous period: $\widehat{\phi}_{i}^{t+1} \equiv \frac{\phi_{i}^{t+1}}{\phi_{i}^{t}} \cdot 30$

We use WIOD data to construct time series of trade shares $\left\{\pi_{k, o i}^{t}\right\}$, sectoral price indices $\left\{P_{k, i}^{I, t}\right\}$ and final good expenditures $\left\{E_{i}^{C, t}\right\}$ between December of 2000 and December of 2014. Armed with these data, we can exploit the gravity structure of the trade block of the model, as in Head and Ries (2001) and Eaton et al. (2016), to recover the changes in bilateral trade costs combining equations equations (28) and (30):

$$
\widehat{d}_{k, o i}^{t}=\frac{\widehat{P}_{k, i}^{I, t}}{\widehat{P}_{k, o}^{I, t}}\left(\frac{\widehat{\pi}_{k, o o}^{t}}{\widehat{\pi}_{k, o i}^{t}}\right)^{1 / \lambda}
$$

In turn, we rely on the Euler equation (35) and normalize $\widehat{\phi}_{U S}^{t}=1 \forall t$, as in Reyes-Heroles (2016), to recover the inter-temporal preference shocks:

$$
\widehat{\phi}_{i}^{t+1}=\frac{E_{i}^{C, t+1}}{E_{i}^{C, t}} \frac{E_{U S}^{C, t}}{E_{U S}^{C, t+1}} \text { for } t=1, \ldots, T-1,
$$

where $T$ is the last period for which we have data, which refers to December of 2014. Note that we still need to determine $\widehat{\phi}_{i}^{T+1}$, but we will need to use the structure of the model to do so, as this value will depend on the model-implied steady-state value for final good expenditures $E_{i}^{C, \infty}$.

Finally, we recover the productivity shocks $\left\{\widehat{A}_{k, i}^{t}\right\}$ using:

$$
\widehat{A}_{k, i}^{t}=\frac{\widehat{\pi}_{k, i i}^{t}}{\left(\widehat{P}_{k, i}^{I, t}\right)^{\lambda}}\left(\widehat{c}_{k, i}^{t}\right)^{\lambda} .
$$

\footnotetext{
${ }^{30}$ We impose $\widehat{A}_{k, i}^{t}=\widehat{A}_{k, i}^{T}$ and $\widehat{d}_{k, o i}^{t}=\widehat{d}_{k, o i}^{T}$ for all $t>T$, where $T$ is the last period for which we have data $(T=14$ and refers to December 2014). We also impose and $\widehat{\phi}_{i}^{t}=1$ for all $t>T+1$-as explained below, the value of $\widehat{\phi}_{i}^{T+1}=1$ is set to gauge the model-implied steady-state value for final consumption expenditures $E_{i}^{C, \infty}$.
} 
Given that $\widehat{c}_{k, i}^{t}$ depends on $\widetilde{w}_{k, i}^{t}$, which has no data counterpart, we need to use the full structure of the model to recover the sequence of productivity shocks. Online Appendix C.6 details the algorithm to recover the shocks $\left\{\widehat{A}_{k, i}^{t}\right\}$ as well as $\left\{\widehat{\phi}_{i}^{T+1}\right\}$ using the full structure of the model. To be able to recover the full set of shocks the economy experienced between 2000 and 2014, we assume the economy faces no additional shocks after 2015. That is, the values for $\left\{A_{k, i}^{t}\right\},\left\{d_{k, o i}^{t}\right\}$ and $\left\{\phi_{i}^{t}\right\}$ are imposed to be constant from 2015 onwards. $^{31}$

Figure 9a shows an increase in productivity all over the world. In particular, China has experienced large increases in productivity, especially in manufacturing sectors. ${ }^{32}$ Other emerging economies - which comprise the bulk of the Americas and the Rest of the World aggregate - also experienced impressive productivity growth, while growth was more muted for advanced economies.

Turning to trade costs, we first construct a summary statistic to capture this large object. We focus on the average import cost for each country-sector pair, weighted by their initial steady state import shares:

$$
\bar{d}_{k, i}^{t}=\sum_{o \neq i} \frac{\pi_{k, o i}^{0}}{1-\pi_{k, i i}^{0}} \widehat{d}_{k, o i}^{t} .
$$

Figure 9b plots this index for each country and sector. In general, import trade costs are declining for the United States and Asia, and approximately flat in Europe (with some heterogeneity across sectors). Perhaps surprisingly, starting after the 2008 financial crisis and concurrent collapse in trade, initially falling import trade costs in China begin to revert and are actually larger by the end of the sample. This estimate of changes in trade costs reflects the fall in the share of trade in output, as documented in Bems et al. (2013). The sources for these increasing frictions are myriad, and include policy changes in countries like China, as well as changes in supply chain management, and other reasons. That said, our measures of frictions are a standard, straightforward, measure of the implied barriers to trade.

Finally, we turn to our measure of shocks to inter-temporal preferences, which are presented in Figure 10. The shocks in the US are normalized to 1 in every period. In Europe and Asia (except China), the discount factor shocks fluctuate around 1, suggesting little persistent deviations in consumption behavior from what would be expected with a simple consumption smoothing model. On the other hand, China, the Americas, and the aggregated remaining countries (Rest of the World) exhibit persistent shocks to their inter-temporal preferences, suggesting increased patience over the period we consider. These persistent deviations are often referred to as the "global savings

\footnotetext{
${ }^{31}$ We also assume the economy is in steady state in 2000 and fully anticipates the full set of current and future shocks in 2001.

${ }^{32}$ While we plot changes in the productivity location parameters $\left\{\widehat{A}_{k, i}^{t}\right\}$, this is not directly comparable to productivity in the classic sense of a Solow Residual. In order to make sense of the magnitudes, note that TFP growth, defined as $\widehat{c}_{k, i}^{t} / \widehat{P}_{k, i}^{I, t}$, can be expressed as $\left(\widehat{A}_{k, i}^{t} / \widehat{\pi}_{k, i i}^{t}\right)^{1 / \lambda}$. Therefore, using our recovered values for $\widehat{A}_{k, i}^{t}$, data on changes in trade shares, and imposing $\lambda=4$, the magnitude for actual annualized TFP growth in China ranges from 3 to $5 \%$ per year, depending on the sector - which is in line with growth accounting estimates discussed in Zhu (2012).
} 
Figure 9: Extracted Globalization Shocks

(a) Productivity Shocks $\widehat{A}_{k, i}^{t}$
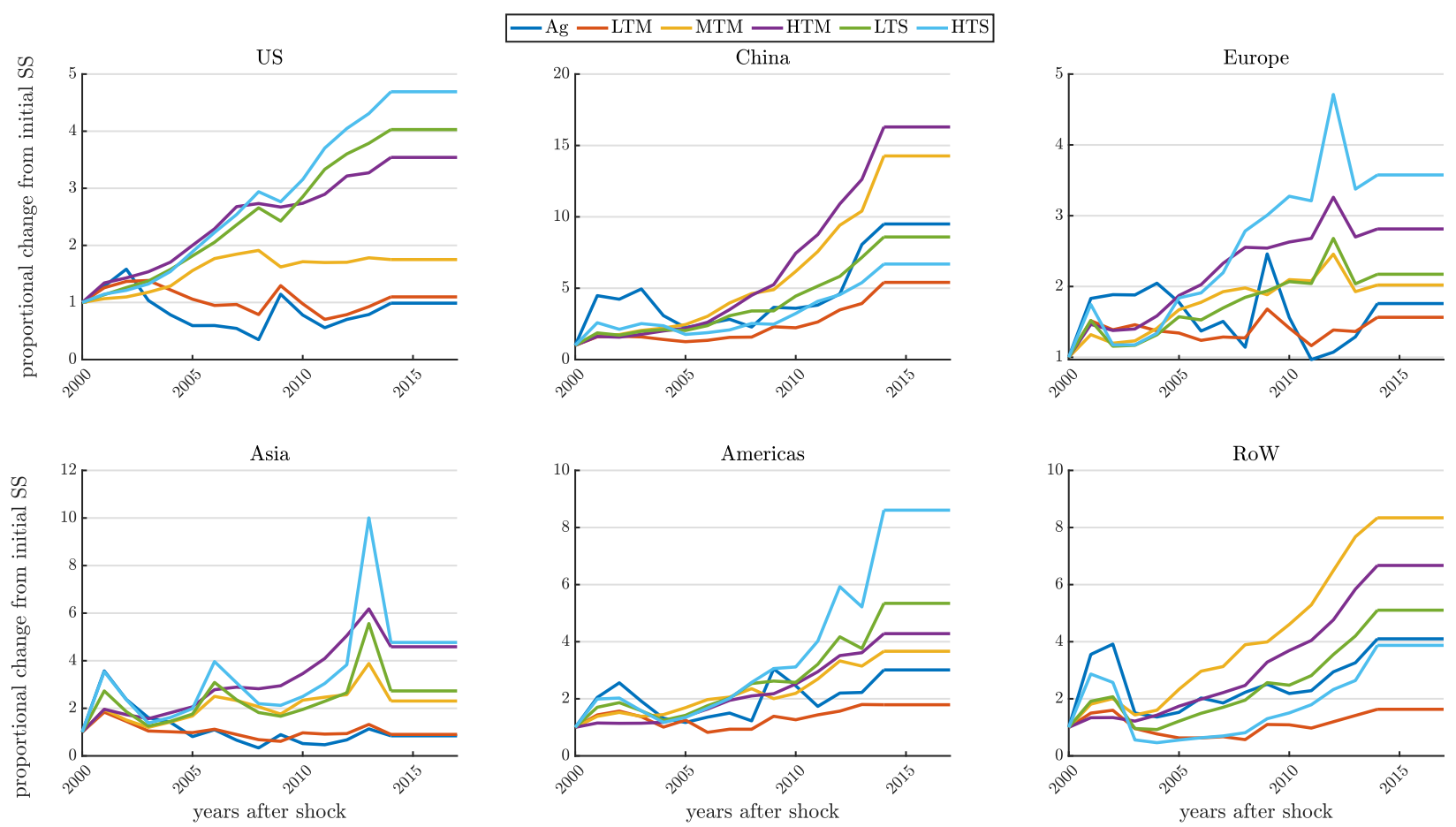

(b) Trade-Weighted Import Costs $\bar{d}_{k, i}^{t}$ (See Equation (42))
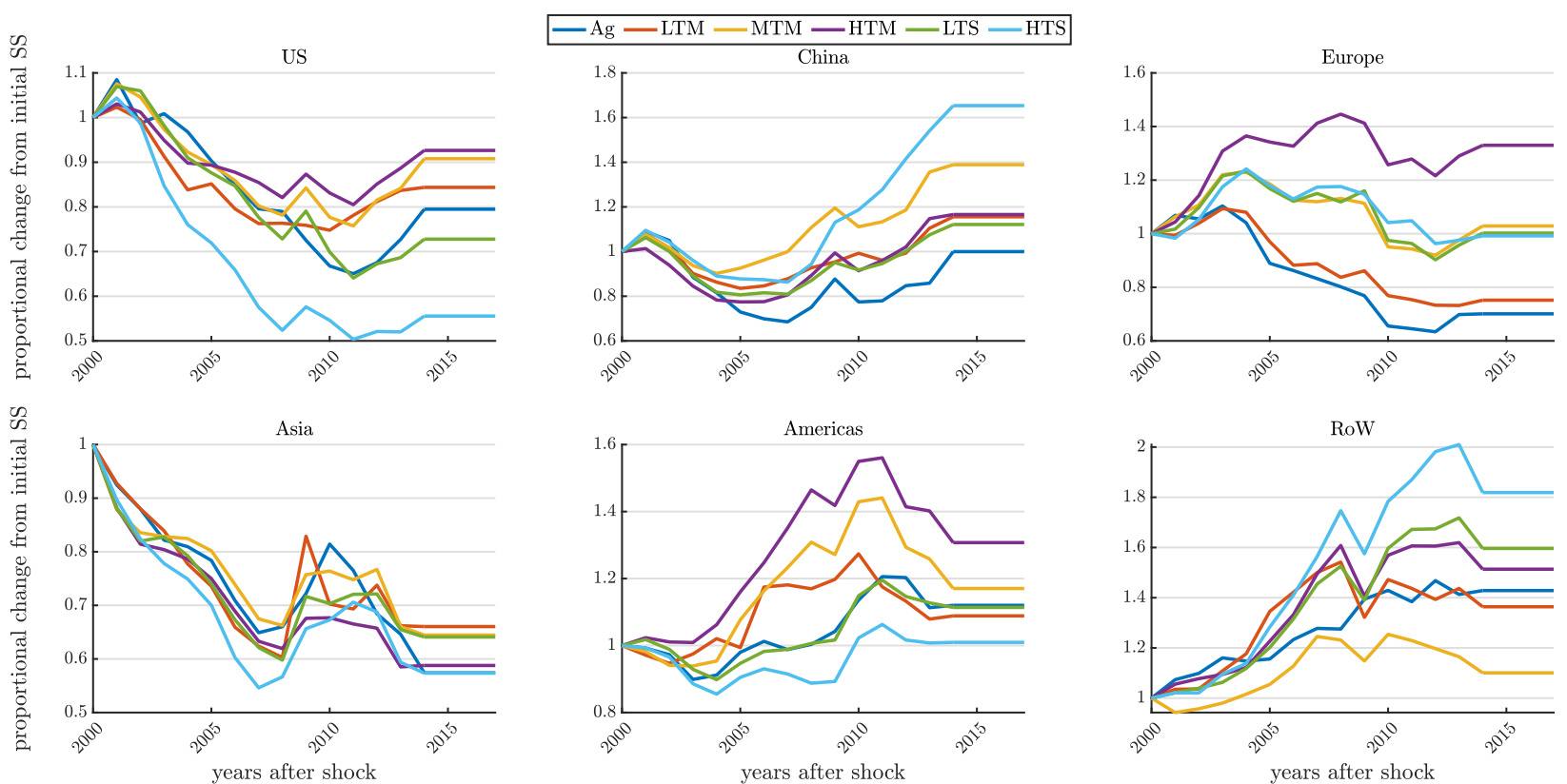
glut." ${ }^{33}$ It is important to recognize that there are rich dynamics to consumption in the real world, reflecting preferences, frictions, and other factors. We are agnostic on the exact theory, instead summarizing the effect of these channels with the $\widehat{\phi}_{i}^{t}$ shocks. This is useful because it allows us to ask counterfactual questions about the dynamics of globalization shocks without the global savings glut, without having to specify what policy or change in deep parameters to achieve this - a useful benchmark to compare against the usual assumption in trade of no consumption smoothing whatsoever.

Figure 10: Extracted Inter-Temporal Preference Shocks $\widehat{\phi}_{i}^{t}$
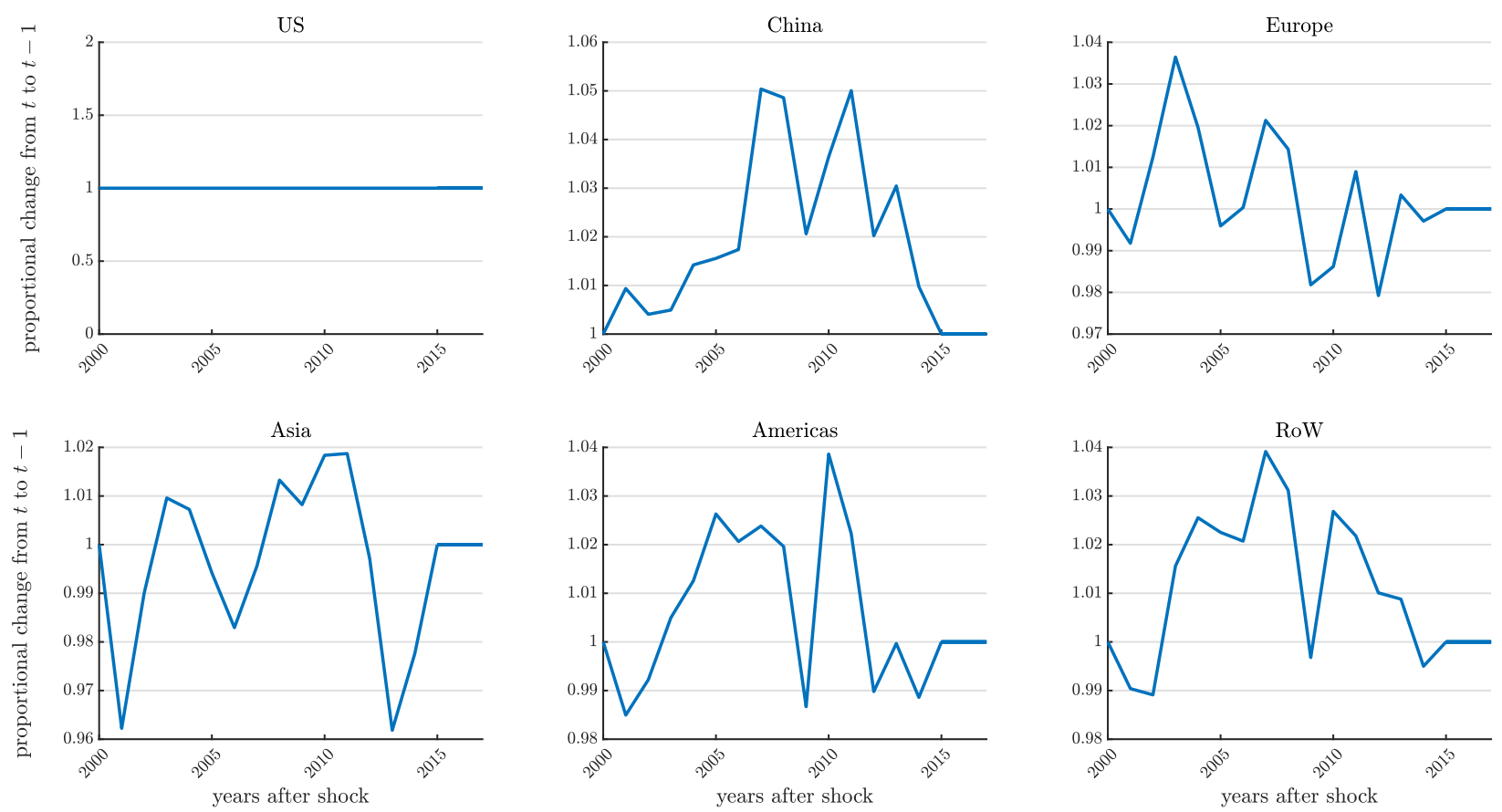

To extract the shocks the global economy experienced between 2000 and 2014, we have solely relied on data on trade shares, sectoral prices, and aggregate final expenditures across countries. We have not used information on labor allocations or trade imbalances. It is therefore natural to ask how the model-implied behavior of labor allocations and trade imbalances compare to those in the data. A note of caution before we proceed with this comparison: given our perfect foresight assumption, once we feed the shocks into the model, responses at impact are large as agents (suddenly) fully anticipate the path of future shocks. That said, Figure 11 compares labor allocations in the United States in our model (Panel a) to labor allocations in the data (Panel b). Our model replicates the decline of manufacturing as a whole, the expansion of services, and the decline and rebound of agriculture. The magnitudes in our model are compressed, but we believe this is explained by

\footnotetext{
${ }^{33}$ The large trade surplus that China has been running since the early 2000s is a puzzle for models in which the main driving forces are productivity shocks. For instance, as argued by Song et al. (2011), financial frictions within China are key drivers of the Chinese savings glut. Our inter-temporal preference shocks constitute a reduced-form way to allow the model to match the time series behavior of Chinese aggregate expenditures and the rest of the world.
} 
pre-existing trends driving the decline of manufacturing as well as the fact that we impose that the inter-temporal shocks $\widehat{\phi}_{i}^{t}$ revert to 1 after 2014. The latter observation is important as individuals in the model anticipate the end of the savings glut years before the end of our sample period, leading to some rebalancing happening before 2014. This observation is also important to explain the strong rebound of Low-Tech Manufacturing, for which we do not find support in the data. To verify the plausibility of this explanation, we simulated the same shocks we extracted with one difference: we keep the $\widehat{\phi}_{i}^{t}$ shocks fixed at their 2012 to 2014 averages for 15 more years (that is, until 2029). Indeed, not only the magnitude of the downsize of manufacturing that we obtain is larger than what is depicted in Figure 11a, but also Low-Tech steadily contracts until 2014.

Figure 11: Comparing Labor Allocations in the Model and Data

(a) Model

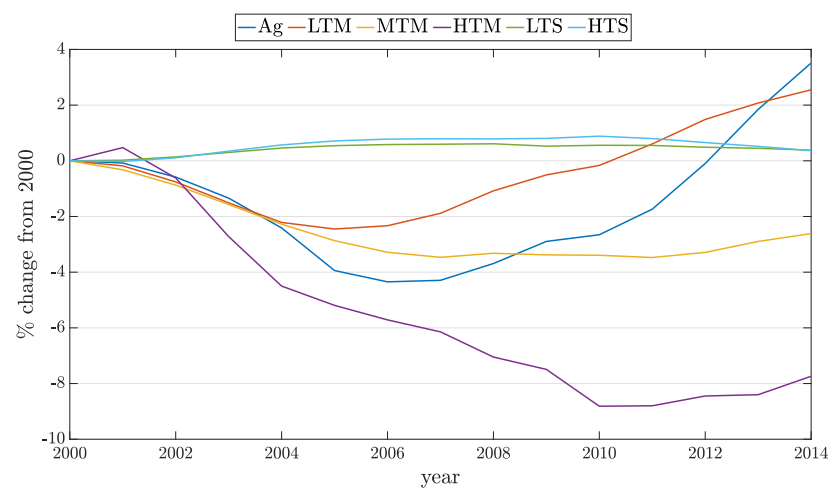

(b) Data

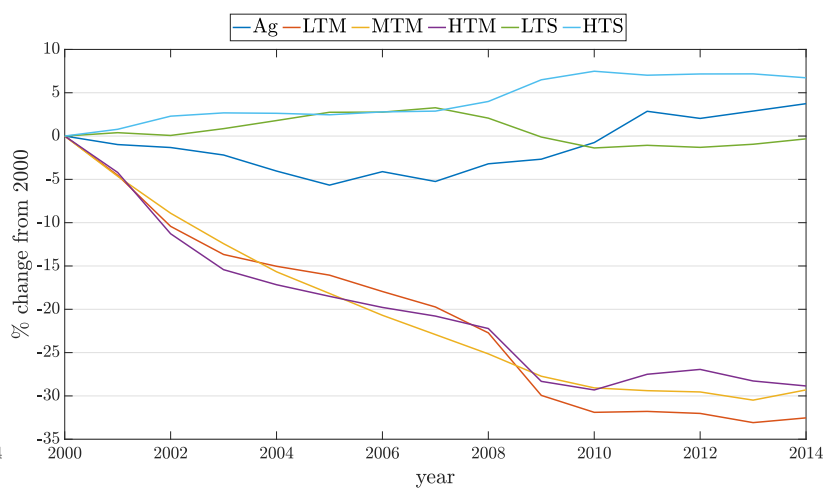

Figure 12 compares the model-implied trade imbalances in the US and China to those in the data. The model is able to capture the large surplus in China as well as the persistent deficit in the US. However, the model misses the behavior of Chinese imbalances in the beginning of the period. This happens as China anticipates its massive shocks that are coming (both large increases in productivity and shocks to inter-temporal preferences), which leads to large unemployment in the short run. In turn, this implies an initial production shortage and trade deficits.

\subsection{Global Technology and Trade Shocks}

Our first counterfactual exercise focuses on the general equilibrium effects of changes in productivity and trade costs since 2000, with no shocks to inter-temporal preferences. In particular, we focus on the consequences of our extracted series of $\left\{\widehat{A}_{i}^{t}\right\}$ and $\left\{\widehat{d}_{k, o i}^{t}\right\}$ above. Our goal is to isolate the quantitative significance of globalization on labor market outcomes in a setting where workers smooth their consumption over time. As we will show, the shocks we recover from the data have significant impacts on global imbalances, which has implications for adjustment dynamics, unemployment, wages, and, ultimately, consumption.

Before discussing results, it is helpful to contrast our approach with standard practice. There 
Figure 12: Comparing Net Exports in the Model and Data

(a) Model

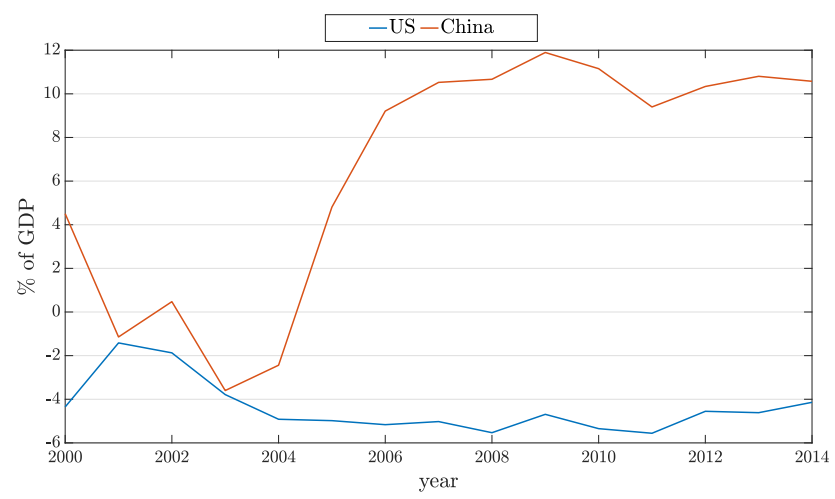

(b) Data

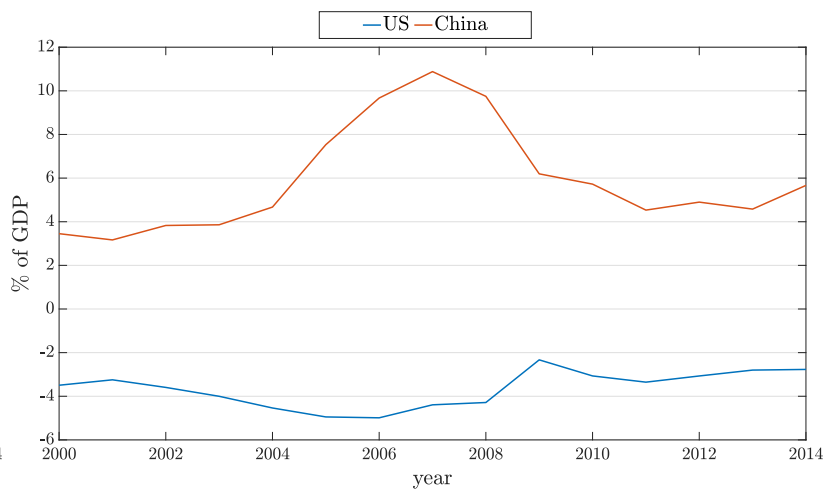

are two typical approaches to dealing with imbalances in the quantitative trade literature. The first approach involves fixing imbalances in the data, or assuming balanced trade period by period. This method has been employed in both static models (e.g., Eaton and Kortum (2002) and Dekle et al. (2007)) and dynamic models (e.g., Dix-Carneiro (2014) and Traiberman (2019)). The use of this method in dynamic models is a particularly strong assumption, since it implicitly imposes that workers and governments have no access to borrowing or savings mechanisms. The second approach, involves assigning ownership shares of capital or fixed factors to agents at an initial point, and fixing these shares over time and in counterfactuals. Although this procedure does not follow from optimizing behavior, this method has been used in Caliendo et al. (2019), allowing for imbalances to change as returns to fixed factors change. If returns to capital increase while those to labor decline, this implies that workers have access to some social insurance. Nevertheless, agents are prevented from buying or selling shares in response to the shocks they face. ${ }^{34}$

To be able to compare the implications of the globalization shocks in our model of trade imbalances relative to a model without imbalances, we start both models from a steady steady with trade balance - that is $N X_{i}^{t}=0 \forall i$ at $t=0$, see Online Appendix C.3 for details on our procedure, which is based on exact hat algebra. Figure 13 shows that the globalization shocks we feed into the model can lead to substantial imbalances in the short run. These predictions range from a trade deficit in China of up to $65 \%$ of GDP to a trade surplus in Asia close to 20\%. The US runs a short-run surplus amounting to close to 10\% of GDP. As expected from previous literature, if we purge the model of the inter-temporal preference shocks, we see China running a large trade deficit in the short run. This is not surprising in light of our discussion in section 4.2 as Chinese productivity strongly and steadily increases between 2000 and 2014, more than in other countries.

\footnotetext{
${ }^{34}$ These approximations may be reasonable in a situation where imbalances are small along the transition path from one equilibrium to the next. This would be the case, for example, if the primary force for changes in imbalances were shocks to aggregate consumption, and orthogonal to trade shocks (i.e., if imbalances were driven by $\widehat{\phi}_{i}^{t}$ ). Nevertheless, these are imperfect if the path and magnitude of trade shocks lead to large changes in consumption and savings, which we show is the case below.
} 
Figure 13: Net Exports Over GDP in Response to Global Technology and Trade Shocks
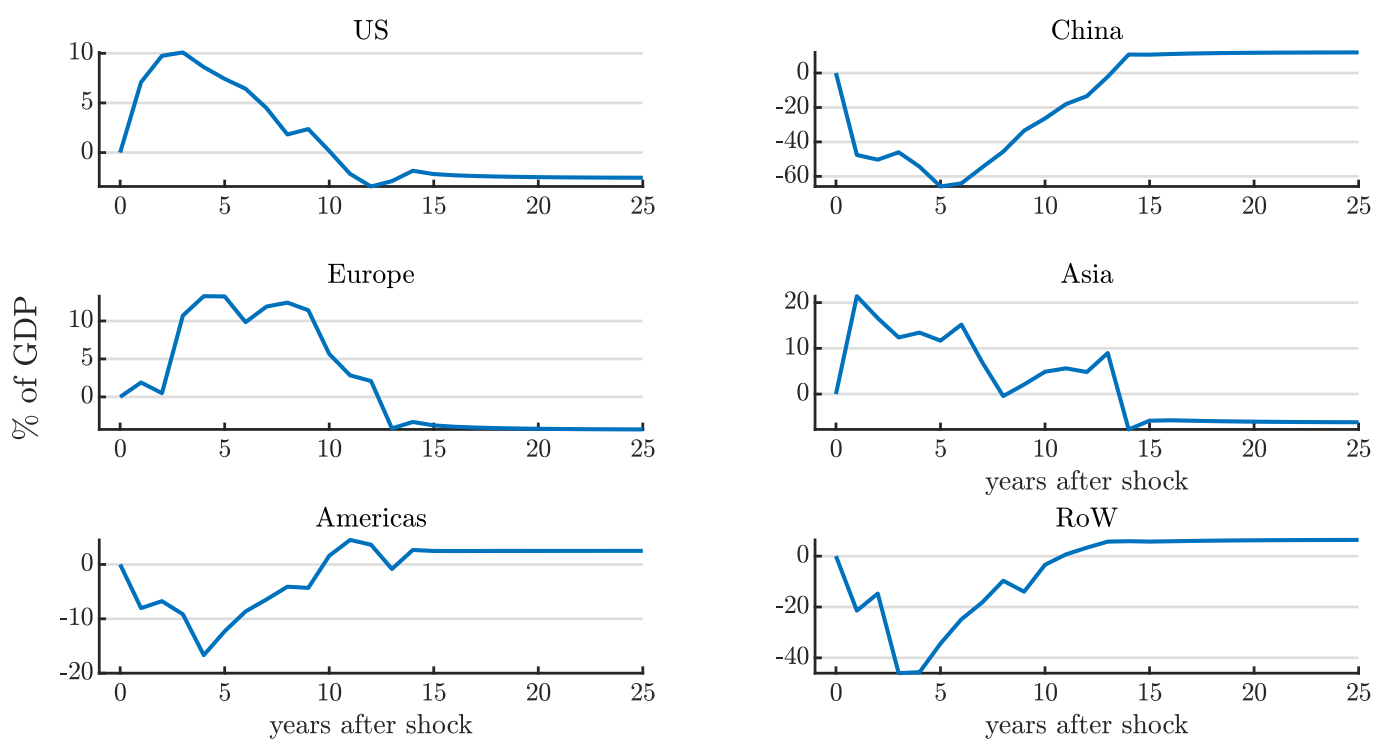

We learned in our analysis of section 4.2 that trade imbalances can amplify the amount of reallocation in the economy relative to a model where trade is balanced. Our empirical exercise corroborates this finding, as we illustrate in Figure 14a. The behavior of unemployment is also quite different, and driven by a larger amount of reallocation. Figures 13 and 14b also show that the responses of unemployment are not systematically related to the sign of imbalances. Importantly, Figures 14c and 14d highlight the importance of modeling trade imbalances in understanding the adjustment process in response to globalization shocks. A model that imposes trade balance often predicts opposing patterns of short-run reallocation. For example, our model with imbalances predicts that the globalization shocks would lead to an expansion of all manufacturing sectors in the US in the short run, but a contraction in the long run. On the other hand, trade balance would lead to a monotonic decline of High-Tech Manufacturing and a long-run expansion of Low-Tech Manufacturing. Other countries also display drastically different patterns of adjustment in both the short and long runs.

\subsection{Trade Costs and Imbalances}

The previous section established that empirically-extracted shocks can lead to significant trade imbalances, and that accounting for these imbalances can substantially alter the adjustment process relative to a balanced-trade world. This section studies the implications of both trade imbalances and labor market frictions for the consumption gains from trade, and for how these compare with the widely used sufficient-statistics approach based on Arkolakis et al. (2012), henceforth ACR. Our model nests the Ricardian model considered in ACR, but violates two of their key assumptions: (i) 
Figure 14: Labor Market Dynamics in Response to Global Technology and Trade Shocks

(a) Reallocation Index
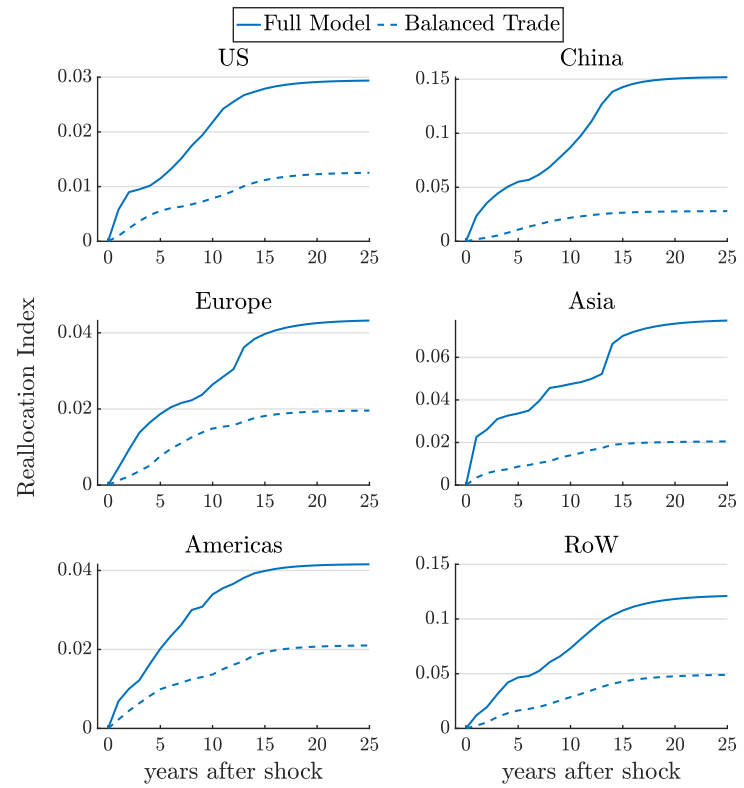

(c) Labor Allocations - Full Model
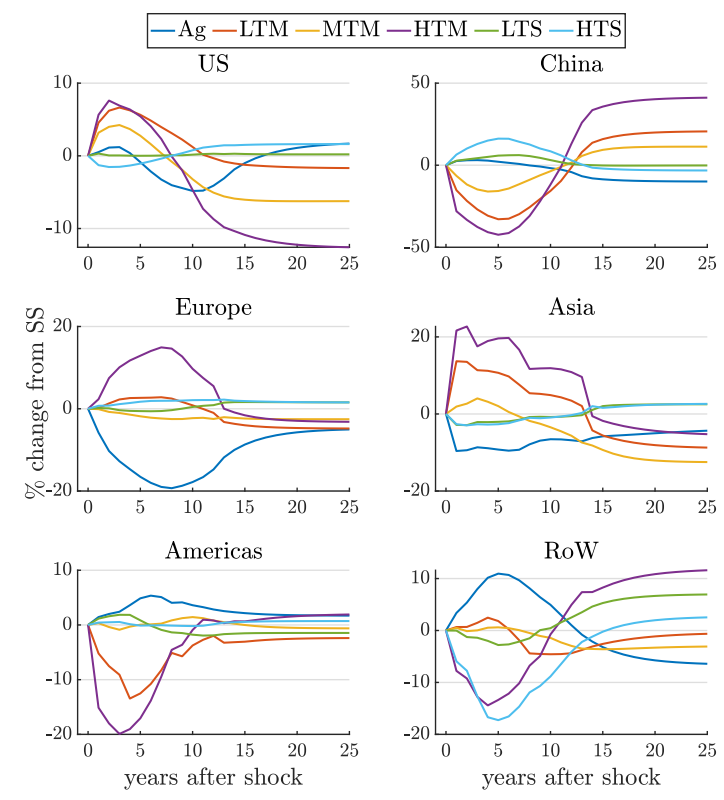

(b) Unemployment

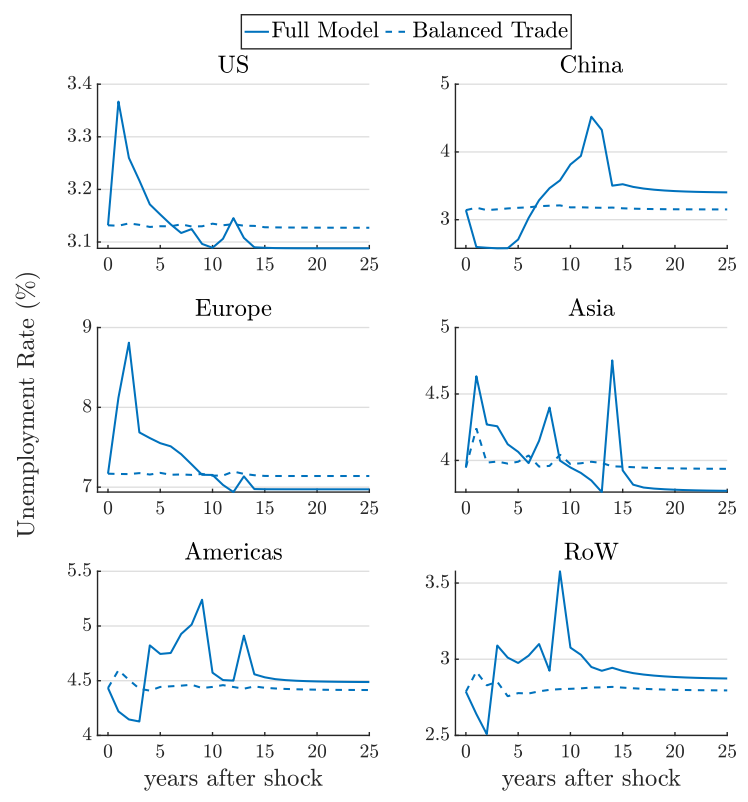

(d) Labor Allocations - Balanced Trade
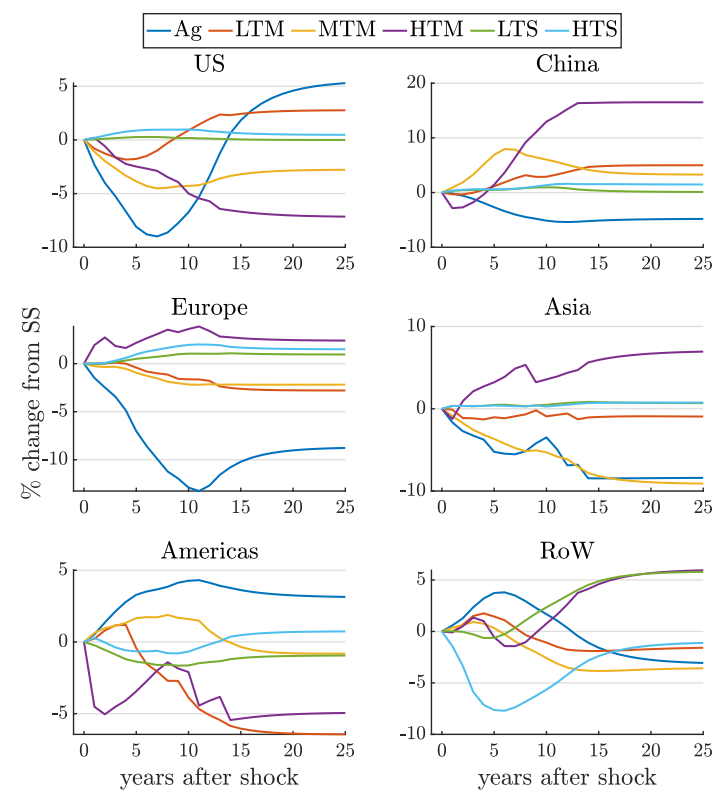
no labor market frictions; and (ii) no trade imbalances.

Concretely, we consider the changes in trade costs between 2000 and 2014 we obtain in Section 5.1 , purging the model of shocks to inter-temporal preferences and to productivity. In this case, the implied gains from trade following Costinot and Rodríguez-Clare (2014), who extend the ACR formula to allow for input-output linkages, is given by:

$$
\widehat{W}_{i}^{\mathrm{ACR}, \text { Static }}=\prod_{j=1}^{K} \prod_{k=1}^{K} \widehat{\pi}_{k, i i}^{-\mu_{j, i} \aleph_{j k, i} / \lambda}
$$

where all of the changes are between final and initial steady states and $\aleph_{j k, i}$ is the $j, k^{\text {th }}$ element of the Leontief Inverse of the input-output matrix in country $i$. We obtain $\widehat{\pi}_{k, i i}$ solving our full model. To perform the comparison between consumption gains in our model and those using the ACR formula, we first focus on the change in steady-state consumption given by our framework. Given the static nature of the ACR model, we believe this is a more direct comparison between our predictions for the gains from trade.

Figure 15a displays the comparison between the long-run ACR gains in consumption (blue bars) and the long-run gains we obtain in our model (red bars). Overall, these gains are quite different. The ACR formula predicts that the US endures a $0.6 \%$ loss in response to the changes in trade costs the global economy experiences, whereas our model predicts that the US experiences a gain of $0.9 \%$. Our conclusions differ starkly in China, where the ACR formula predicts a gain of almost $3 \%$, but our model predicts a long-run loss of $0.7 \%$. These numbers differ because of both labor market frictions and long-run trade imbalances that arise in our model. The latter figures depend on the full path of shocks fed into the model, and not just the initial and final levels of trade costs - as do the ACR gains. Figure 16 shows the long-run trade imbalances that arise in our model. They are particularly important in China, Asia, and the Rest of the World.

Given the dynamic nature of our model, we define the dynamic consumption gains from trade as the ratio between the level of constant consumption that would yield the same net present value consumption that unfolds along the transition path and the initial steady-state consumption. Mathematically:

$$
\widehat{W}_{i} \equiv \exp \left\{(1-\delta) \sum_{t=0}^{\infty} \delta^{t} \log \left(C_{i}^{t}\right)-\log \left(C_{i}^{S S_{0}}\right)\right\} .
$$

Similarly, we can also calculate "ACR Dynamic" gains from trade, by taking the net present value of the static gains calculated by (43) in every period. More precisely:

$$
\widehat{W}_{i}^{\mathrm{ACR}, \text { Dynamic }}=(1-\delta) \sum_{t=0}^{\infty} \delta^{t}\left[\prod_{j=1}^{K} \prod_{k=1}^{K}\left(\widehat{\pi}_{k, i i}^{t}\right)^{-\mu_{j, i} \aleph_{j k, i} / \lambda}\right]
$$


where $\widehat{\pi}_{k, i i}^{t}$ is the change in trade shares between periods 0 and $t$, computed using our full model.

Figure 15: Shocks in Trade Costs and the Consumption Gains from Trade
(a) Long-Run Gains from Trade
(b) Dynamic Gains from Trade
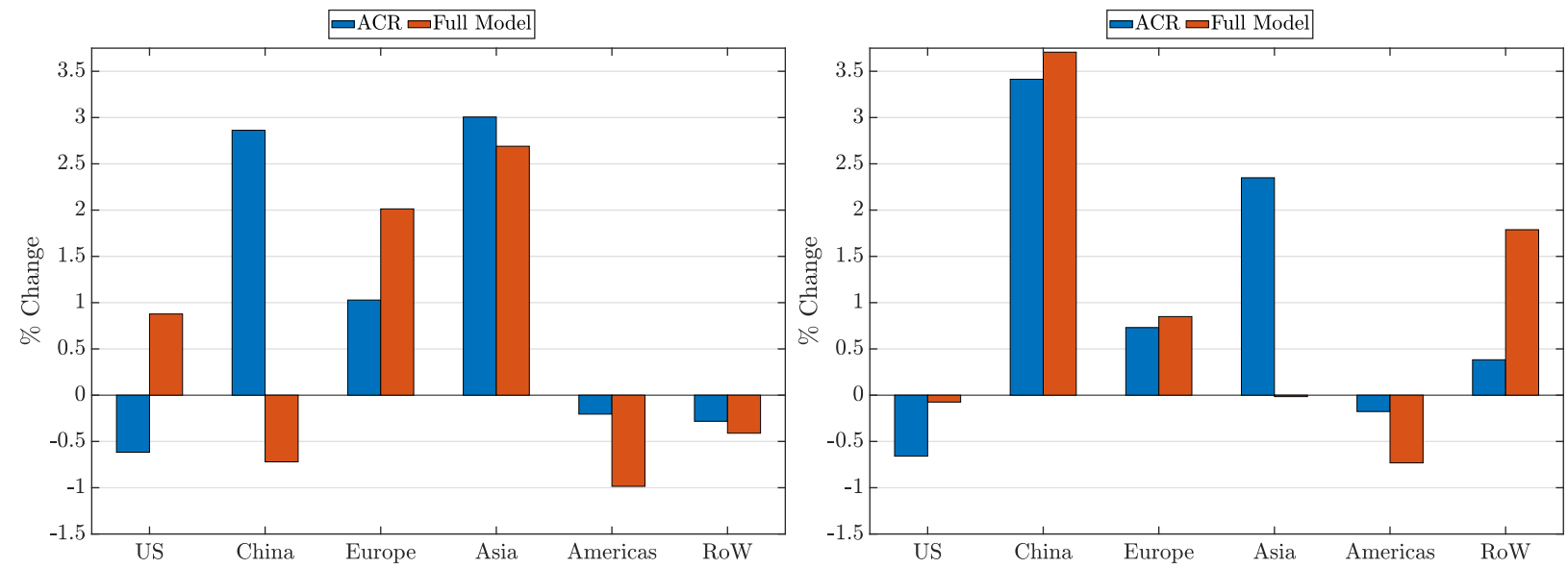

Notes: In Panel (a), the blue bars, "ACR," refer to the consumption gains computed using equation (43); the red bars, "Full Model," refer to the change in steady-state consumption given by our full model with trade imbalances. In Panel (b), the blue bars, "ACR," refer to the present value of gains calculated by using equation (45); the red bars, "Full Model," refer to the present value of gains over the transition in the full model with trade imbalances, using equation (44). In all cases, $\widehat{\pi}_{k, i i}^{t}$ is obtained by simulating our full model initialized with $N X_{i}^{t}=0 \forall i$ at $t=0$

Figure 15b compares the consumption gains predicted by the "dynamic ACR" formula (45) to the dynamic gains computed according our model (44). Although predictions are now similar for China and Europe, they are quite different for the remaining countries. For example, Asia enjoys a consumption gain of almost $2.5 \%$ according to the dynamic ACR formula, whereas our model predicts an essentially zero gain. Also noteworthy, the Rest of the World gains by almost $2 \%$ according to our model, but by less than $0.5 \%$ according to the dynamic ACR formula. In a series of exercises available upon request, we investigate the separate role of trade imbalances and labor market frictions behind the discrepancies between the predictions of the ACR formula and those of our model. To that end, we simulate our model under balanced-trade and still find significant differences between the gains predicted by our model and those by the ACR formula. We conclude that both trade imbalances and labor market frictions are important contributors to the divergences we document.

\subsection{Globalization Gains}

The previous section compared how the consumption gains in our model compare with those from the ACR formula in response to shocks in trade costs alone. We now ask by how much countries benefitted from all globalization shocks during the period we consider, which include shocks to trade costs, technology and inter-temporal preferences. To that aim, we adopt the following procedure. For each country at a time, we neutralized the shocks it experienced between 2000 and 2014, while 
Figure 16: Steady-Sate Changes in Net Exports in Response to Shocks in Trade Costs

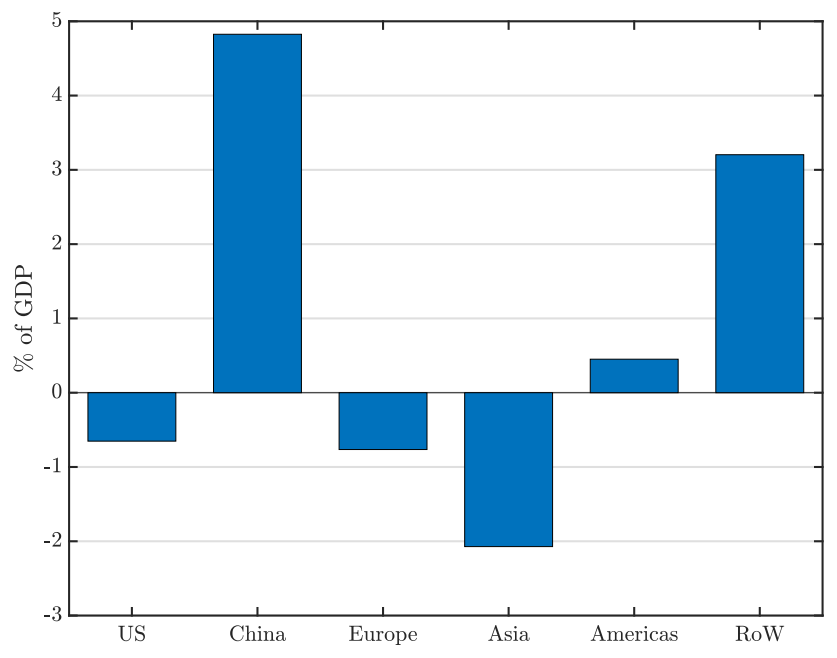

feeding the model with the path of shocks faced by the remaining countries. For example, to assess the effects of globalization on US consumption, we set all US shocks to 1, but we keep shocks to remaining countries at the values we recovered in Section 5.1. Table V displays the results. The first column shows that all countries benefitted from globalization. China benefitted the most, with consumption gains reaching 6.6\%. The Americas and Asia/Oceania gained the least: $0.03 \%$. The magnitude of gains can be significantly affected if we impose trade balance: the second column shows that gains in the US are reduced from $2.2 \%$ to $1.3 \%$. Finally, we investigate the effect of intertemporal shocks in the third and fourth columns (with and without trade imbalances, respectively). If we shut down the global savings glut, welfare in the US is significantly higher, reaching $3.8 \%$. We conclude observing that even though our results are consistent with the belief that the global savings glut was detrimental to the US, balancing trade would lead to an even worse situation.

\subsection{The China Shock}

As a final application of our model, we investigate the role of China on the adjustment of the labor market in the US. This topic has attracted much academic interest since the work of Autor et al. (2013) and Pierce and Schott (2016). We compare the behavior of our model when we feed it with all of the shocks we recovered in Section 5.1 (which we label as the Benchmark counterfactual) to the behavior of the model when (a) shocks to China are set to be equal to the average of shocks experienced by the remaining countries; and when (b) we neutralize China's savings glut by setting $\widehat{\phi}_{\text {China }}^{t}=1$ for all $t .{ }^{35}$ In these simulations, trade imbalances in the initial steady state are set at $N X_{i}^{t=0}=N X_{i}^{D a t a}$, that is, the level of trade imbalances in the data in 2000.

\footnotetext{
${ }^{35}$ Shocks to inter-temporal preferences and their interaction with standard shocks in productivities and trade costs differentiate these counterfactual experiments from Caliendo et al. (2019) and Adão et al. (2020).
} 
Table V: Globalization Consumption Gains Over 2000-2014

\begin{tabular}{lcccc}
\hline \hline Country & Complete Model & Balanced Trade & $\begin{array}{c}\text { Complete Model } \\
\widehat{\phi}_{i}^{t}=1 \forall i, t\end{array}$ & $\begin{array}{c}\text { Balanced Trade } \\
\widehat{\phi}_{i}^{t}=1 \forall i, t\end{array}$ \\
\hline United States & 1.022 & 1.013 & 1.038 & 1.013 \\
China & 1.066 & 1.067 & 1.056 & 1.067 \\
Europe & 1.015 & 1.015 & 1.025 & 1.016 \\
Asia/Oceania & 1.003 & 1.021 & 0.997 & 1.021 \\
Americas & 1.003 & 1.006 & 1.002 & 1.006 \\
Rest of the World & 1.052 & 1.045 & 1.037 & 1.045 \\
\hline \hline
\end{tabular}

Notes: Each row displays $\widehat{W}_{i}$ (see equation (44)) in response to extracted shocks to all other countries when own country shocks are neutralized. Third and fourth columns impose $\widehat{\phi}_{i}^{t}=1 \forall i, t$, neutralizing all inter-temporal preference shocks. To make the Complete Model exercises comparable with the Balanced Trade ones, we initialize the Complete Model with $N X_{i}^{t}=0$ for $t=0$.

We first analyze the situation where shocks to China between 2000 and 2014 are imposed to be equal to the average of shocks across all of the remaining countries. ${ }^{36}$ The objective is to understand the behavior of the global economy in a counterfactual world where Chinese fundamentals evolved like those of an average country. The red dashed line in Figure 17a shows that China runs a much more modest trade surplus over time compared to the Benchmark illustrated by the blue solid line. Interestingly, Figure 17b shows that the behavior of the US trade deficit is barely affected if shocks to China are set to other countries' averages. This result suggests that the evolution of the US trade deficit between 2000 and 2014 was not greatly influenced by the extraordinary shocks China experienced over this period. Instead, the evolution of the trade deficit in the US was primarily dictated by the full constellation of shocks that the global economy experienced over the 2000's. This result highlights an important advantage of our multi-country model relative to two-country models, where changes in the trade balance in one country are mirrored in the other.

Figure 18 describes the impact of the China shock on sectoral reallocation in the US. The red dashed line shows that if shocks to China had behaved in an ordinary way between 2000 and 2014, the contraction in Mid- and High-Tech Manufacturing would have been less pronounced. The effect of the China shock on employment in Low-Tech Manufacturing is not as visible, but the dashed red line is consistently above the solid blue line, showing that shocks to the Chinese economy led to mild contractions in employment in the sector (or prevented stronger growth in the long run). In contrast, employment in Agriculture and Services (particularly Low-Tech Services) moved in opposite directions, quickly absorbing workers displaced from Manufacturing. Figure 19a shows that the behavior of unemployment with the China shock (solid blue line) or without it (dashed

\footnotetext{
${ }^{36}$ Shocks to trade costs from country $i$ to China are set to be equal to a weighted average of shocks between country $i$ and all other countries - weights are given by country sizes $\bar{L}_{i}$. Shocks to trade costs from China to country $i$ are set to be equal to the weighted average of shocks between all remaining countries and country $i$.
} 
Figure 17: The China Shock: Net Exports

(a) NX / GDP in the China

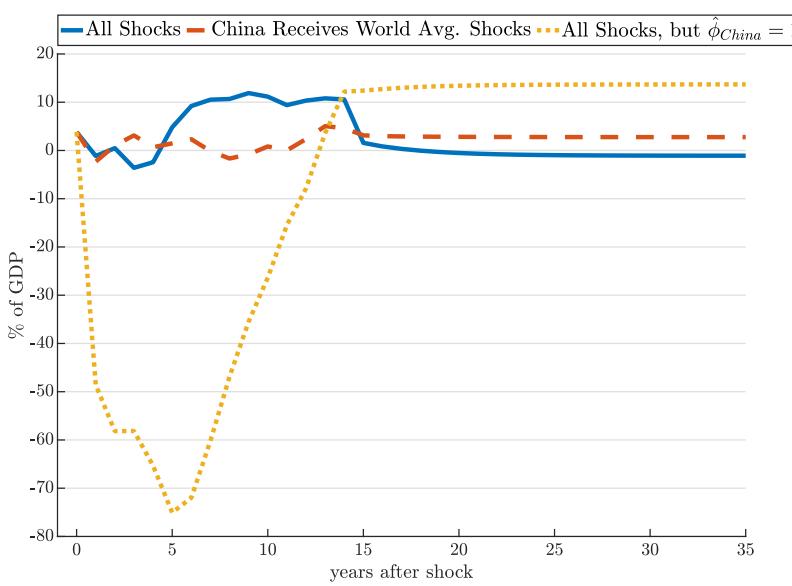

(b) NX / GDP in the US

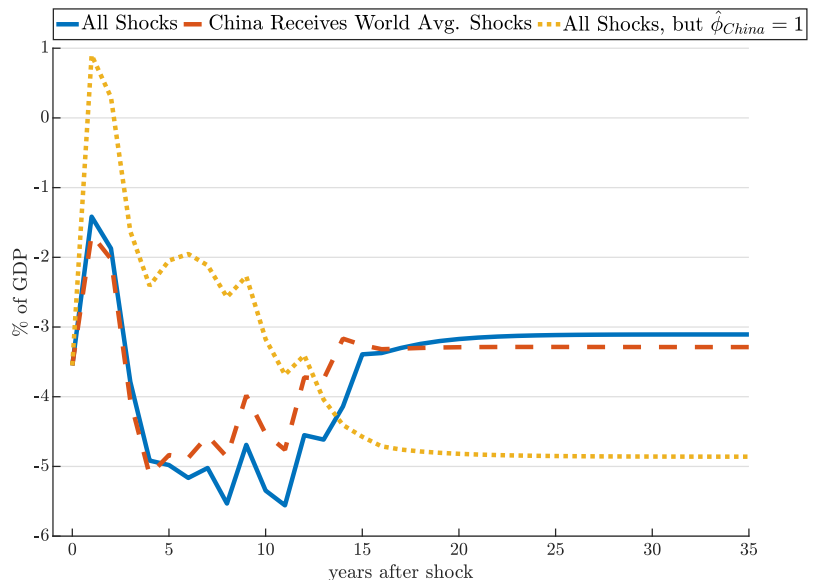

Notes: "All Shocks": model is fed with all shocks recovered in section 5.1. "China Receives World Avg. Shocks": model is fed with all shocks recovered in section 5.1, with the exception of China. For China, productivity and inter-temporal productivity shocks between 2000 and 2014 are imposed to be equal to the average of these shocks across all the remaining countries. Shocks to trade costs from country $i$ to China are set to be equal to a weighted average of shocks between country $i$ and all other countries - weights are given by country sizes $\bar{L}_{i}$. Shocks to trade costs from China to country $i$ are set to be equal to the weighted average of shocks between all remaining countries and country $i$. "All Shocks but $\widehat{\phi}_{\text {China }}=1$ ": model is fed with all shocks recovered in section 5.1 but China's savings glut by setting $\widehat{\phi}_{\text {China }}^{t}=1$ for all $t . t=0$ corresponds to year 2000. $t=14$ corresponds to 2014 , the last year of data we employed to extract the shocks.

red line) is very similar. Indeed, in either case, the unemployment rate reaches a maximum of $3.14 \%$.

It is important to highlight that the small response of US unemployment in Figure 19a is not a mechanical feature of our model. Indeed, Figures 5b, 8b and $14 \mathrm{~b}$ show substantial changes in the unemployment rate in the US and other countries in response to other shocks we simulate. Further insights can be obtained if we study the behavior of sectoral unemployment. Consider the "All Shocks" counterfactual, where we feed the model with all the shocks we extracted in Section 5.1. Figure 19b shows that the response of sectoral unemployment rates is considerably larger than the aggregate response. Specifically, aggregate unemployment ranges from 3.06\% to $3.14 \%$ in response to the extracted shocks. At the sectoral level, unemployment within the manufacturing sectors repond more strongly and range between $3.65 \%$ and $4.35 \%$ and unemployment within agriculture varies between $2.6 \%$ and $3.4 \%$. However, unemployment within the service sectors are essentially unresponsive to the shocks. Therefore, given that $86 \%$ of workers are initially in services, the effect of these shocks on US unemployment is muted. On the other hand, aggregate unemployment ranges from $6.5 \%$ to $12.5 \%$ in Europe and from $3.5 \%$ and $14 \%$ in China, showing that the model is capable of producing substantial unemployment responses to global shocks.

It is also instructive to compare the behavior of the model when it is subjected to all the global shocks we extracted in Section 5.1 (the Benchmark) to its behavior when we subject it with the 
Figure 18: The China Shock: Labor Allocations in the US
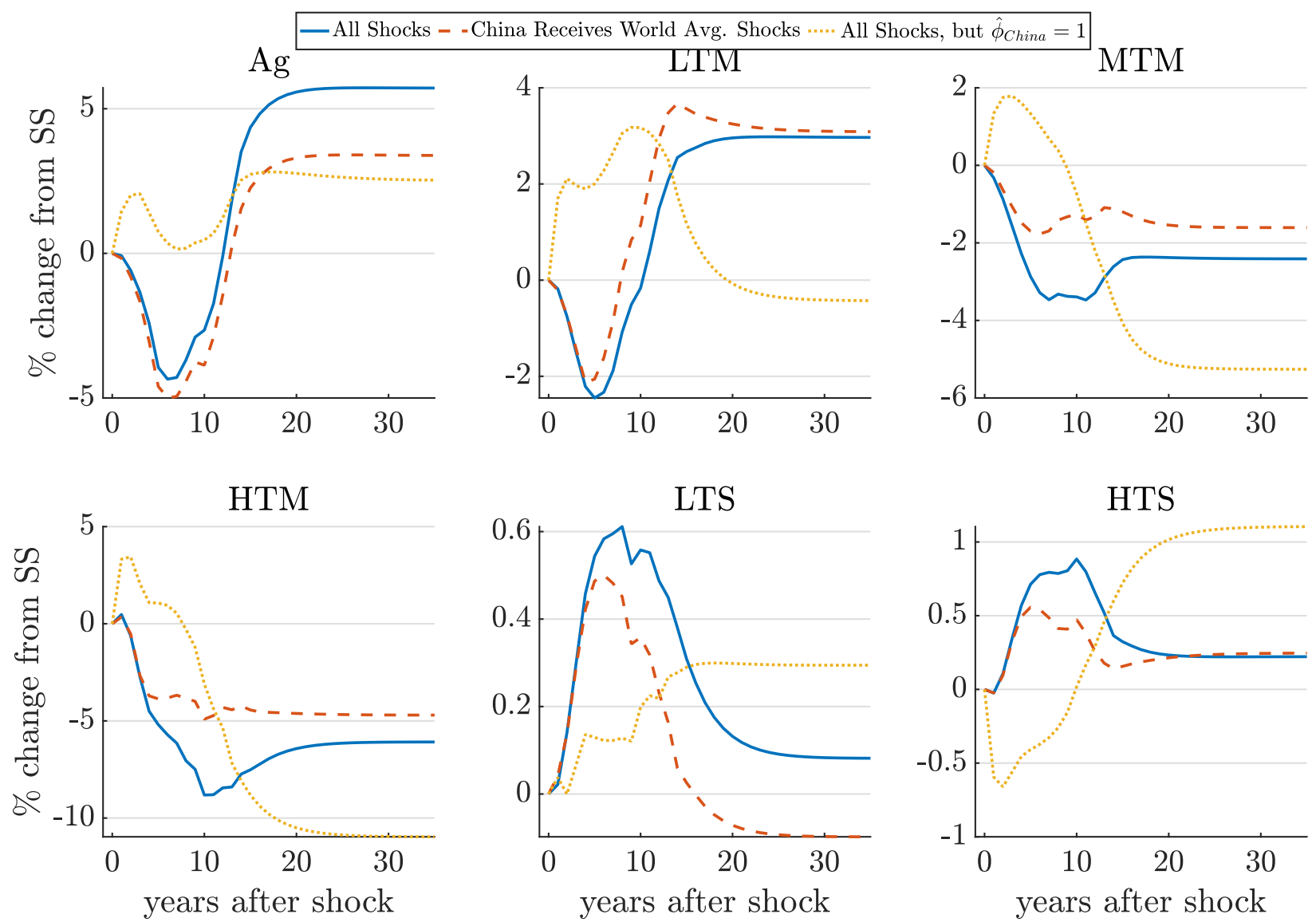

Notes: "All Shocks": model is fed with all shocks recovered in section 5.1. "China Receives World Avg. Shocks": model is fed with all shocks recovered in section 5.1, with the exception of China. For China, productivity and inter-temporal productivity shocks between 2000 and 2014 are imposed to be equal to the average of these shocks across all the remaining countries. Shocks to trade costs from country $i$ to China are set to be equal to a weighted average of shocks between country $i$ and all other countries - weights are given by country sizes $\bar{L}_{i}$. Shocks to trade costs from China to country $i$ are set to be equal to the weighted average of shocks between all remaining countries and country $i$. "All Shocks but $\widehat{\phi}_{\text {China }}=1$ ": model is fed with all shocks recovered in section 5.1 but China's savings glut by setting $\widehat{\phi}_{\text {China }}^{t}=1$ for all $t$. $t=0$ corresponds to year 2000. $t=14$ corresponds to 2014 , the last year of data we employed to extract the shocks.

same shocks but remove China's saving glut (that is, we set $\widehat{\phi}_{\text {China }}^{t}=1$ for all $t$ ). The yellow dotted line in Figure 17a shows that, in the absence of China's savings glut, China would have run a massive trade deficit in the short run, reaching $75 \%$ of GDP. ${ }^{37}$ These large short-run deficits are then accompanied by large permanent trade surpluses in the long run, surpassing 10\% of GDP. The evolution of the dotted yellow line is unsurprising in light of our our discussion in Section 4.2, and, more specifically, given Figure 7. In anticipation of a much higher level of productivity in the future (see Figure 9a), China borrows greatly in the short run to smooth consumption over time.

\footnotetext{
${ }^{37}$ In our model, in the absence of shocks to inter-temporal preferences, countries perfectly smooth consumption expenditure over time. This fact gives rise to a surge in imbalances that is not in line with what we observe in the data. There is ample evidence that the world is far from perfect consumption smoothing or risk sharing as documented and analyzed in Heathcote and Perri (2014).
} 
Figure 19: The China Shock: Unemployment in the US

(a) Aggregate Unemployment

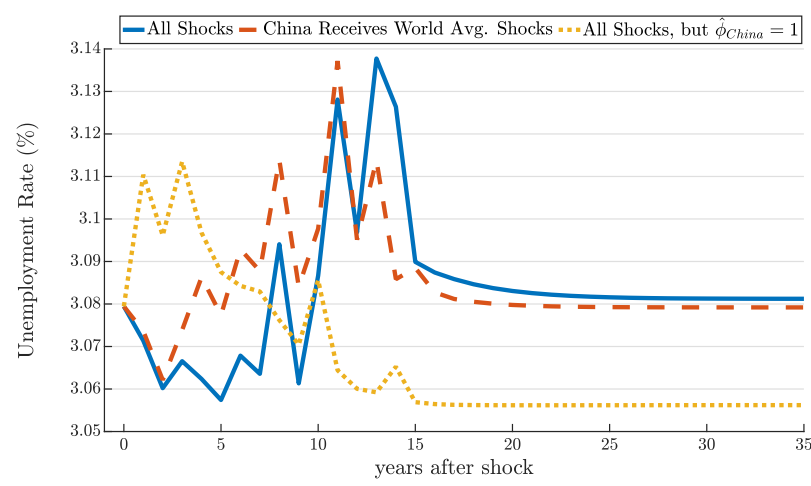

(b) Sectoral Unemployment

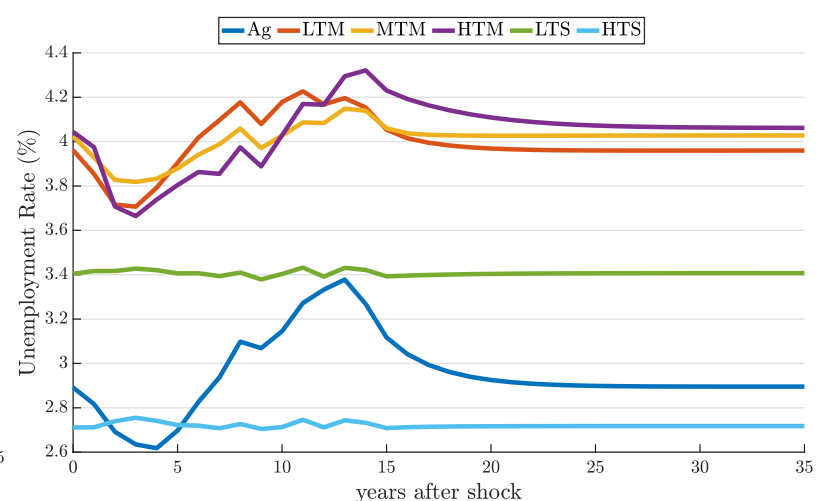

Notes: Panel (a) — "All Shocks": model is fed with all shocks recovered in section 5.1. "China Receives World Avg. Shocks": model is fed with all shocks recovered in section 5.1, with the exception of China. For China, productivity and inter-temporal productivity shocks between 2000 and 2014 are imposed to be equal to the average of these shocks across all the remaining countries. Shocks in trade costs from country $i$ to China are set to be equal to the simple average of shocks between country $i$ and all other countries. Shocks to trade costs from country $i$ to China are set to be equal to a weighted average of shocks between country $i$ and all other countries-weights are given by country sizes $\bar{L}_{i}$. Shocks to trade costs from China to country $i$ are set to be equal to the weighted average of shocks between all remaining countries and country $i$. "All Shocks but $\widehat{\phi}_{\text {China }}=1$ ": model is fed with all shocks recovered in section 5.1 but China's savings glut by setting $\widehat{\phi}_{\text {China }}^{t}=1$ for all $t$. $t=0$ corresponds to year 2000. $t=14$ corresponds to 2014, the last year of data we employed to extract the shocks. Panel (b) - Behavior of sectoral unemployment rates in response to "All Shocks".

This debt must be repaid in the form of large surpluses in the long run. This result illustrates the quantitatively important role of shocks to inter-temporal preferences $\widehat{\phi}_{\text {China }}^{t}$ not only in offsetting these large short-run trade deficits, but also in turning them into surpluses. ${ }^{38}$

Neutralizing the Chinese savings glut also has important effects on the dynamics of the US trade deficit. The yellow dotted line in Figure 17b shows that the US trade deficit would be significantly smaller in the short run. However, the trade deficit would stabilize at a larger value in the long run (5\% of GDP) as China runs a permanent large trade surplus. This result highlights that, even in the absence of the China's savings glut, consumption smoothing motives in the global economy would lead to a large trade surplus in China in the long run (north of 10\% of GDP) as the trade deficit in the US deteriorates to $5 \%$ of GDP.

The dotted yellow and solid blue lines in Figure 18 show interesting consequences of China's savings glut on the size of the manufacturing sector in the US. The Chinese savings glut was responsible for large declines in US manufacturing in the short run. This behavior is consistent across all three manufacturing sectors. However, absent the Chinese savings glut, manufacturing employment in the US would be substantially lower in the long run as China runs a permanently large trade surplus, and the US runs a permanently large trade deficit. ${ }^{39}$

\footnotetext{
${ }^{38}$ If we abstract from intertemporal preference shocks in China, our result is in line with the "allocation puzzle" in open economy macroeconomics which states that aggregate net capital inflows tend to be negatively correlated with productivity growth across developing countries (Gourinchas and Rey, 2014). In line with this puzzle, our intertemporal preference shifters could be interpreted as arising from the public sector's expenditure decisions (Gourinchas and Jeanne, 2013; Aguiar and Amador, 2011; Alfaro et al., 2008).

${ }^{39}$ These results are in line with the findings of Kehoe et al. (2018) on the effects of the savings glut on structural
} 
We now turn to the welfare consequences of the China shock. Specifically, we use equation (44) to compute the welfare effects of the shocks we recover in Section 5.1 (Benchmark), relative to the counterfactuals where (a) shocks to China are set to be equal to the average shocks experienced by the remaining countries: $\widehat{W}_{i}^{\text {Benchmark }} / \widehat{W}_{i}^{(a)}$; and where (b) we neutralize China's savings glut by setting $\widehat{\phi}_{\text {China }}^{t}=1$ for all $t: \widehat{W}_{i}^{\text {Benchmark }} / \widehat{W}_{i}^{(b)}$. By doing so, we are capturing the effect of China's shocks relative to a situation where the Chinese fundamentals behaved similarly to the average global economy (excluding China), and relative to a situation where China did not experience a savings glut.

Table VI: Global Consumption Gains of the China Shock (2000-2014)

\begin{tabular}{lcc}
\hline \hline & \multirow{2}{*}{ Panel A. $\widehat{W}_{i}^{\text {Benchmark }} / \widehat{W}_{i}^{(a)}$} \\
\hline Country & Complete Model & Balanced Trade \\
\hline US & 1.001 & 1.012 \\
Europe & 1.001 & 1.003 \\
Asia & 1.004 & 0.994 \\
Americas & 1.001 & 1.001 \\
Rest of the World & 1.005 & 1.031 \\
\hline
\end{tabular}

\begin{tabular}{lcc} 
& Panel B. $\widehat{W}_{i}^{\text {Benchmark }} / \widehat{W}_{i}^{(b)}$ \\
\hline Country & Complete Model & Balanced Trade \\
\hline US & 0.997 & 1.000 \\
Europe & 0.998 & 1.000 \\
Asia & 0.998 & 1.000 \\
Americas & 1.003 & 1.000 \\
Rest of the World & 1.010 & 1.000 \\
\hline
\end{tabular}

Notes: Changes in welfare obtained using equation (44). $\widehat{W}_{i}^{\text {Benchmark }}$ refers to all shocks extracted in Section 5.1 fed into the model. $\widehat{W}_{i}^{(a)}$ refers to these same shocks, but China's shocks at set to the average of shocks in remaining countries. $\widehat{W}_{i}^{(b)}$ refers to all shocks extracted in Section 5.1 fed into the model, but $\widehat{\phi}_{\text {China }}^{t}=1$ for all $t$. To make the Complete Model exercises comparable with the Balanced Trade ones, we initialize the Complete Model with $N X_{i}^{t}=0$ for $t=0$.

Panel A of Table VI shows the effect of the China shock relative to a situation where China experienced shocks set to the average of the remaining countries. The first column displays the consumption effects of the China shock in our complete model with trade imbalances. The second column shows the consumption effects if we impose trade balance period by period. First, note that all countries benefitted from the extraordinary shocks accrued to China. However, these consumption effects were all very small. Interestingly, the welfare effects of the China shock in the change in the US. 
US and the Rest of the World would be significantly higher if trade balanced every period. Panel B shows the effect of the Chinese savings glut. Our model predicts that the US, Europe, and Asia were negatively affected by the large shocks to inter-temporal preferences in China, but that these effects were, again, very mild. The exception is the Rest of the World, that actually benefits from the Chinese savings glut. The Chinese savings glut would have had virtually no consumption effects around the world under balanced trade.

\section{Conclusion}

There is a widespread concern among policy makers that, as globalization brings disruption to the labor market, growing trade deficits can accentuate job losses and the decline of manufacturing. Given how persistent these concerns have been in the past four decades, it is surprising that trade economists typically abstract from trade imbalances when they study the labor market consequences of globalization shocks. This paper fills this gap by extending the workhorse model of trade (Eaton and Kortum, 2002, and Caliendo and Parro, 2015) to allow for inter-sectoral mobility costs (as in Artuç et al., 2010), search frictions (as in Mortensen and Pissarides, 1999), and trade imbalances (as in Reyes-Heroles, 2016). In short, we extend the state-of-the-art model of trade with the workhorse models for mobility frictions, unemployment and trade imbalances. We estimate the model using data from the WIOD, ILOSTAT, and the US CPS. Our estimation method conditions on trade shares and net exports and can be performed country by country. Even though the parameter space is large, the country by country estimation allows us to break a large optimization problem into smaller ones.

After analyzing impulse response functions to hypothetical shocks, we find that: (i) incorporating trade imbalances has the potential to significantly magnify the effect of globalization shocks on inter-sectoral reallocation and unemployment; (ii) reallocation paths can be substantially different if we allow for trade imbalances compared to balanced trade - specifically, sectors that expand in the short run with trade imbalances can instead contract with balanced trade; (iii) the behavior of trade imbalances and of the labor market depends on the whole path of shocks; (iv) the response of unemployment to globalization shocks is not related to sign of imbalances. In particular, temporary shocks can have important persistent long-run effects in our model, but none if we impose balanced trade. This motivates us to empirically extract the path of shocks the global economy actually experienced between 2000 and 2014 using standard methods from the international trade and macro literatures.

We conduct a standard exercise in the international trade literature and subject the model to the empirically-extracted global technology and trade cost shocks. Aligned with our impulse response function analyses, we find that allowing for trade imbalances have a first order effect on 
the behavior of labor markets around the world. Next, we investigate how the gains from trade in our model compare with those obtained using the influential sufficient-statistics approach pioneered by Arkolakis et al. (2012). We find that both trade imbalances and labor market frictions can lead to substantial discrepancies between the gains from trade in our model relative to those in the literature on quantitative models of trade, which is summarized in Costinot and Rodríguez-Clare (2014). Not only there are differences in the magnitude of the gains, but also on their signs. Relatedly, we assess, for each country, the gains from globalization between 2000 and 2014. Our model predicts that the US has enjoyed a gain of $2.2 \%$ in response to globalization shocks over this period. Our estimates suggest that these gains would have been $73 \%$ larger in the absence of the global savings glut. However, in a world where balanced trade is imposed, the US would have experienced $40 \%$ smaller gains.

We also use our model to investigate the impact of the "China Shock" on the US trade deficit and labor market. Consonant with previous literature, we find that shocks to the Chinese economy contributed to the decline of manufacturing in the US, especially Mid-Tech and High-Tech Manufacturing. However, other sectors of the economy quickly absorbed workers from these sectors, leading to small effects on unemployment. We also find that shocks to the Chinese economy had only a modest effect on the evolution of the US trade deficit, highlighting that the evolution of the US trade deficit was mostly driven by the full constellation of shocks the global economy experienced. Interestingly, if China had not experienced its savings glut, the US trade deficit would have been smaller in the short run, but larger in the long run.

Our work shows that carefully modeling imbalances can have quantitatively important implications for the adjustment process in response to globalization shocks and opens important questions for future work. Given the importance of imbalances for the reallocation process, a natural question to address next is to quantitatively characterize how trade imbalances shape the inequality effects of trade. Our model can be extended to allow heterogeneous workers, so this is a natural next step. In addition, we also hope to be able to add endogenous capital accumulation decisions and capitalskill complementarity, generating rich mechanisms linking globalization to the skill premium as in Reyes-Heroles et al. (2020). Finally, an interesting extension of our framework would allow workers to make borrowing and savings decisions at the individual level, which will aggregate into global imbalances. Even though this is a hard problem, especially regarding estimation, we believe that our method of simulated moments that can be performed country by country (conditional on trade shares and imbalances) can be applied to this situation. 


\section{References}

Adão, Rodrigo, Costas Arkolakis, and Federico Esposito, "General Equilibrium Effects in Space: Theory and Measurement," Technical Report 2020.

Aguiar, Mark and Manuel Amador, "Growth in the Shadow of Expropriation *," The Quarterly Journal of Economics, 06 2011, 126 (2), 651-697.

Alessandria, George A and Horag Choi, "The dynamics of the US trade balance and real exchange rate: The J curve and trade costs?," Technical Report, National Bureau of Economic Research 2019.

Alfaro, Laura, Sebnem Kalemli-Ozcan, and Vadym Volosovych, "Why Doesn't Capital Flow from Rich to Poor Countries? An Empirical Investigation," The Review of Economics and Statistics, 2008, 90 (2), 347-368.

Arkolakis, Costas, Arnaud Costinot, and Andrés Rodríguez-Clare, "New trade models, same old gains?," American Economic Review, 2012, 102 (1), 94-130.

Artuç, Erhan and John McLaren, "Trade policy and wage inequality: A structural analysis with occupational and sectoral mobility," Journal of International Economics, 2015, 97 (2), $278-294$.

Artuç, Erhan, Shubham Chaudhuri, and John McLaren, "Trade Shocks and Labor Adjustment: A Structural Empirical Approach," American Economic Review, June 2010, 100 (3), $1008-45$.

Autor, David H., David Dorn, and Gordon H. Hanson, "The China Syndrome: Local Labor Market Effects of Import Competition in the United States," American Economic Review, October 2013, 103 (6), 2121-68.

_ , _, and _, "The China Shock: Learning from Labor-Market Adjustment to Large Changes in Trade," Annual Review of Economics, 2016, 8 (1), 205-240.

Bai, Yan and José-Víctor Ríos-Rull, "Demand Shocks and Open Economy Puzzles," American Economic Review, May 2015, 105 (5), 644-49.

Barany, Zsofia, Nicholas Coeurdacier, and Stéphane Guibaud, "Capital Flows in an Aging World," 2018.

Bems, Rudolfs, Robert C. Johnson, and Kei-Mu Yi, "The Great Trade Collapse," Annual Review of Economics, 2013, 5 (1), 375-400.

Bernanke, Benjamin S., "The Global Saving Glut and the U.S. Current Account Deficit," Speech presented at the Sandridge Lecture, Virginia Association of Economists, Richmond, VA, March 102005.

Caballero, Ricardo J., Emmanuel Farhi, and Pierre-Olivier Gourinchas, "An Equilibrium Model of "Global Imbalances" and Low Interest Rates," American Economic Review, March 2008, 98 (1), 358-93.

Caliendo, Lorenzo and Fernando Parro, "Estimates of the Trade and Welfare Effects of NAFTA," Review of Economic Studies, 2015, 82 (1), 1-44. 
_ , Maximiliano Dvorkin, and Fernando Parro, "Trade and Labor Market Dynamics: General Equilibrium Analysis of the China Trade Shock," Econometrica, 2019, 87 (3), 741-835.

Carrère, Céline, Anja Grujovic, and Frédéric Robert-Nicoud, "Trade and Frictional Unemployment in the Global Economy," Journal of the European Economic Association, 012020. jvz074.

Coşar, A. Kerem, Nezih Guner, and James Tybout, "Firm Dynamics, Job Turnover, and Wage Distributions in an Open Economy," American Economic Review, March 2016, 106 (3), $625-63$.

Costa, Francisco, Jason Garred, and João Paulo Pessoa, "Winners and losers from a commodities-for-manufactures trade boom," Journal of International Economics, 2016, 102, 50 -69 .

Costinot, Arnaud and Andrés Rodríguez-Clare, "Trade Theory with Numbers: Quantifying the Consequences of Globalization," in "Handbook of International Economics," Vol. 4, Elsevier, 2014, pp. 197-261.

Dauth, Wolfgang, Sebastian Findeisen, and Jens Suedekum, "Adjusting to Globalization in Germany," IZA DP No. 11299, IZA 2018.

Dekle, Robert, Jonathan Eaton, and Samuel Kortum, "Unbalanced Trade," American Economic Review, May 2007, 97 (2), 351-355.

_ , _ , and _ , "Global rebalancing with gravity: Measuring the burden of adjustment," IMF Staff Papers, 2008, 55 (3), 511-540.

Dix-Carneiro, Rafael, "Trade Liberalization and Labor Market Dynamics," Econometrica, 2014, 82 (3), 825-885.

- and Brian K. Kovak, "Trade Liberalization and Regional Dynamics," American Economic Review, October 2017, 107 (10), 2908-46.

_ and _ , "Margins of labor market adjustment to trade," Journal of International Economics, $2019,117,125-142$.

Dornbusch, R., S. Fischer, and P. A. Samuelson, "Comparative Advantage, Trade, and Payments in a Ricardian Model with a Continuum of Goods," The American Economic Review, 1977, 67 (5), 823-839.

Eaton, Jonathan and Samuel Kortum, "Technology, Geography, and Trade," Econometrica, 2002, 70 (5), 1741-1779.

_ , _, and Brent Neiman, "On Deficits and Unemployment," Revue économique, 2013, 64 (3), 405-420.

_ , _ , , and John Romalis, "Trade and the Global Recession," American Economic Review, November 2016, 106 (11), 3401-38.

Flinn, Christopher J., "Minimum Wage Effects on Labor Market Outcomes under Search, Matching, and Endogenous Contact Rates," Econometrica, 2006, 74 (4), 1013-1062. 
Gourinchas, Pierre-Olivier and Hélène Rey, "Chapter 10 - External Adjustment, Global Imbalances, Valuation Effects," in Gita Gopinath, Elhanan Helpman, and Kenneth Rogoff, eds., Handbook of International Economics, Vol. 4 of Handbook of International Economics, Elsevier, 2014, pp. $585-645$.

- and Olivier Jeanne, "Capital Flows to Developing Countries: The Allocation Puzzle," The Review of Economic Studies, 02 2013, 80 (4), 1484-1515.

Guner, Nezih, Alessandro Ruggieri, and James Tybout, "Training, Offshoring, and the Job Ladder," Technical Report, Pennsylvania State University 2020.

Hagedorn, Marcus and Iourii Manovskii, "The Cyclical Behavior of Equilibrium Unemployment and Vacancies Revisited," American Economic Review, September 2008, 98 (4), 1692-1706.

Head, Keith and John Ries, "Increasing Returns versus National Product Differentiation as an Explanation for the Pattern of U.S.-Canada Trade," American Economic Review, September 2001, 91 (4), 858-876.

Heathcote, Jonathan and Fabrizio Perri, "Chapter 9 - Assessing International Efficiency," in Gita Gopinath, Elhanan Helpman, and Kenneth Rogoff, eds., Handbook of International Economics, Vol. 4 of Handbook of International Economics, Elsevier, 2014, pp. 523 - 584.

Heid, Benedikt and Mario Larch, "Gravity with unemployment," Journal of International Economics, 2016, 101, $70-85$.

Helpman, Elhanan and Oleg Itskhoki, "Labour Market Rigidities, Trade and Unemployment," The Review of Economic Studies, 07 2010, 77 (3), 1100-1137.

_ and _ , "Trade Liberalization and Labor Market Dynamics with Heterogeneous Firms," Technical Report 2015.

Hornstein, Andreas, Per Krusell, and Giovanni L. Violante, "Frictional Wage Dispersion in Search Models: A Quantitative Assessment," American Economic Review, December 2011, 101 (7), 2873-98.

Ju, Jiandong, Kang Shi, and Shang-Jin Wei, "On the connections between intra-temporal and intertemporal trades," Journal of International Economics, 2014, 92, S36 - S51. 36th Annual NBER International Seminar on Macroeconomics.

Kambourov, Gueorgui, "Labour Market Regulations and the Sectoral Reallocation of Workers: The Case of Trade Reforms," The Review of Economic Studies, 10 2009, 76 (4), 1321-1358.

Kehoe, Timothy J and Kim J Ruhl, "Sudden stops, sectoral reallocations, and the real exchange rate," Journal of Development Economics, 2009, 89 (2), 235-249.

Kehoe, Timothy J., Kim J. Ruhl, and Joseph B. Steinberg, "Global Imbalances and Structural Change in the United States," Journal of Political Economy, 2018, 126 (2), 761-796.

Krugman, Paul, "Globalization: What Did We Miss?," in Luis Catao and Maury Obstfeld, eds., Meeting Globalization's Challenges, Princeton University Press, 2019, pp. 113-120.

Meghir, Costas, Renata Narita, and Jean-Marc Robin, "Wages and Informality in Developing Countries," American Economic Review, April 2015, 105 (4), 1509-46. 
Mendoza, Enrique G., Vincenzo Quadrini, and José-Víctor Ríos-Rull, "Financial Integration, Financial Development, and Global Imbalances," Journal of Political Economy, 2009, $117(3), 371-416$.

Meza, Felipe and Carlos Urrutia, "Financial liberalization, structural change, and real exchange rate appreciations," Journal of International Economics, 2011, 85 (2), 317-328.

Mortensen, Dale T. and Christopher A. Pissarides, "Job Creation and Job Destruction in the Theory of Unemployment," The Review of Economic Studies, 07 1994, 61 (3), 397-415.

_ and _ , "Unemployment Responses to 'Skill-Biased' Technology Shocks: The Role of Labour Market Policy," The Economic Journal, 1999, 109 (455), 242-265.

Navarro, Peter, "A Tariff Issue on Which Free and Fair Traders Can Agree," Wall Street Journal, May 2019.

Obstfeld, Maurice and Kenneth Rogoff, "The Intertemporal Approach to the Current Account," Handbook of International Economics, 1995, 3, 1731-99.

_ and _, "Global current account imbalances and exchange rate adjustments," Brookings papers on economic activity, 2005, 2005 (1), 67-146.

Pavcnik, Nina, "The Impact of Trade on Inequality in Developing Countries," Working Paper 23878, National Bureau of Economic Research September 2017.

Pierce, Justin R. and Peter K. Schott, "The Surprisingly Swift Decline of US Manufacturing Employment," American Economic Review, July 2016, 106 (7), 1632-62.

Reyes-Heroles, Ricardo, "The Role of Trade Costs in the Surge of Trade Imbalances," Technical Report 2016.

_ , Sharon Traiberman, and Eva Van Leemput, "Emerging Markets and the New Geography of Trade: The Effects of Rising Trade Barriers," IMF Economic Review, 2020, 68, 456-508.

Rodriguez-Clare, Andres, Mauricio Ulate, and Juan Pablo Vasquez, "New Keynesian Trade: Understanding the Employment and Welfare Effects of Sectoral Shocks," Technical Report, University of California at Berkeley 2020.

Ruggieri, Alesandro, "Trade and labour market institutions: A tale of two liberalizations," Research Paper 2019/15, University of Nottingham 2019.

Scott, Robert E., "Causes of the Trade Deficit and Its Implications for the U.S. Economy: Hearing Before the Committee on Finance, US Senate," 105th Congress. Testimony of Robert E. Scott 1998.

Shimer, Robert, "The Cyclical Behavior of Equilibrium Unemployment and Vacancies," American Economic Review, March 2005, 95 (1), 25-49.

Simonovska, Ina and Michael E Waugh, "The elasticity of trade: Estimates and evidence," Journal of International Economics, 2014, 92 (1), 34-50.

Song, Zheng, Kjetil Storesletten, and Fabrizio Zilibotti, "Growing Like China," American Economic Review, February 2011, 101 (1), 196-233. 
Stockman, Alan C. and Linda L. Tesar, "Tastes and Technology in a Two-Country Model of the Business Cycle: Explaining International Comovements," The American Economic Review, 1995, 85 (1), 168-185.

Traiberman, Sharon, "Occupations and Import Competition: Evidence from Denmark," American Economic Review, December 2019, 109 (12), 4260-4301.

Utar, Hale, "Workers beneath the Floodgates: Low-Wage Import Competition and Workers' Adjustment," The Review of Economics and Statistics, 2018, 100 (4), 631-647.

Zhu, Xiaodong, "Understanding China's Growth: Past, Present, and Future," Journal of Economic Perspectives, November 2012, 26 (4), 103-24. 


\section{Online Appendix, Not for Publication}

\section{A Decentralizing the Labor Supply Decision in the Household Problem}

This Section shows that the labor supply decision solving (6) subject to (7) and (5) can be decentralized to individual workers solving equations (5) and (9).

The Lagrangian of problem (6), (7) and (5) is

$$
\mathcal{L}=E_{0}\left\{\begin{array}{c}
\sum_{t=0}^{\infty}\left[\delta^{t} \phi^{t}\left(\bar{L} u\left(c_{\ell}^{t}\right)-\widetilde{\lambda}^{t}\left(\bar{L} P^{F, t} c_{\ell}^{t}+B^{t+1}-\Pi^{t}-R^{t} B^{t}\right)\right)\right]+ \\
\int_{0}^{\bar{L}}\left[\sum_{t=0}^{\infty}\left[\delta^{t} \phi^{t}\left(\begin{array}{c}
\left(1-e_{\ell}^{t}\right)\left(-C_{k_{\ell}^{t}, k_{\ell}^{t+1}}+\omega_{\ell, k_{\ell}^{t+1}}^{t}+b_{k_{\ell}^{t+1}}\right)+ \\
e_{\ell}^{t} \eta_{k_{\ell}^{t}}+\widetilde{\lambda}^{t}\left(\sum_{k=1}^{K} \mathcal{I}\left(k_{\ell}^{t+1}=k\right) e_{\ell}^{t} w_{k}^{t}\left(x_{\ell}^{t}\right)\right)
\end{array}\right)\right]\right] d \ell
\end{array}\right\}
$$

Because each worker is infinitesimal, and the allocation of one worker does not interfere with the allocation/utility of other individual workers (conditional on aggregates), maximizing

$$
\int_{0}^{\bar{L}}\left[\sum_{t=0}^{\infty} \delta^{t} \phi^{t}\left(\begin{array}{c}
\left(1-e_{\ell}^{t}\right)\left(-C_{k_{\ell}^{t}, k_{\ell}^{t+1}}+\omega_{\ell, k_{\ell}^{t+1}}^{t}+b_{k_{\ell}^{t+1}}\right)+ \\
e_{\ell}^{t} \eta_{k_{\ell}^{t}}+\tilde{\lambda}^{t}\left(\sum_{k=1}^{K} \mathcal{I}\left(k_{\ell}^{t+1}=k\right) e_{\ell}^{t} w_{k}^{t}\left(x_{\ell}^{t}\right)\right)
\end{array}\right)\right] d \ell
$$

means maximizing each individual term. Therefore, the planner solves, for each individual, the recursive problem:

$$
\mathcal{L}_{W}^{t}\left(k_{\ell}^{t}, e_{\ell}^{t}, x_{\ell}^{t}, \omega_{\ell}^{t}\right)=\max _{k_{\ell}^{t+1}, \widetilde{e}_{k}^{t+1}(.)}\left\{\begin{array}{c}
\left(1-e_{\ell}^{t}\right)\left(-C_{k_{\ell}^{t}, k_{\ell}^{t+1}}+\omega_{\ell, k_{\ell}^{t+1}}^{t}+b_{k_{\ell}^{t+1}}\right)+e_{\ell}^{t} \eta_{k_{\ell}^{t}} \\
+\widetilde{\lambda}_{t} \sum_{k=1}^{K} \mathcal{I}\left(k_{\ell}^{t}=k\right) e_{\ell}^{t} w_{k}^{t}\left(x_{\ell}^{t}\right)+ \\
\delta \widehat{\phi}^{t+1} E_{t} \mathcal{L}_{W}^{t+1}\left(k_{\ell}^{t+1}, e_{\ell}^{t+1}, x_{\ell}^{t+1}, \omega_{\ell}^{t+1}\right)
\end{array}\right\},
$$

where $\widehat{\phi}^{t+1} \equiv \frac{\phi^{t+1}}{\phi^{t}}$.

Denote by $\mathcal{F}^{t}$ the set of information at $t$. So, $E_{t}()=.E\left(. \mid \mathcal{F}^{t}\right)$. For an unemployed worker in sector $k$ at time $t, k_{\ell}^{t}=k, e_{\ell}^{t}=0$ :

$$
\mathcal{L}_{W}^{t}\left(k_{\ell}^{t}=k, e_{\ell}^{t}=0, x_{\ell}^{t}, \omega_{\ell}^{t}\right)=\max _{k^{\prime},\left\{\tilde{e}_{k}^{t+1}(.)\right\}}-C_{k k^{\prime}}+\omega_{\ell, k^{\prime}}^{t}+b_{k^{\prime}}+\delta \widehat{\phi}^{t+1} E_{t} \mathcal{L}_{W}^{t+1}\left(k^{\prime}, e_{\ell}^{t+1}, x_{\ell}^{t+1}, \omega_{\ell}^{t+1}\right) .
$$


Using the law of iterated expectations we obtain:

$$
\begin{aligned}
& \mathcal{L}_{W}^{t}\left(k_{\ell}^{t}=k, e_{\ell}^{t}=0, x_{\ell}^{t}, \omega_{\ell}^{t}\right)=\max _{k^{\prime},\left\{\widetilde{e}_{k}^{t+1}(.)\right\}}-C_{k k^{\prime}}+\omega_{\ell, k^{\prime}}^{t}+b_{k^{\prime}} \\
& +\delta \widehat{\phi}^{t+1} E\left\{E\left[\mathcal{L}_{W}^{t+1}\left(k^{\prime}, 1, x_{\ell}^{t+1}, \omega_{\ell}^{t+1}\right) \mid x_{\ell}^{t+1}, \mathcal{F}^{t}\right] \times \operatorname{Pr}\left(k_{\ell}^{t+1}=k^{\prime}, e_{\ell}^{t+1}=1 \mid x_{\ell}^{t+1}, \mathcal{F}^{t}\right) \mid \mathcal{F}^{t}\right\} \\
& +\delta \widehat{\phi}^{t+1} E\left\{E\left[\mathcal{L}_{W}^{t+1}\left(k^{\prime}, 0, x_{\ell}^{t+1}, \omega_{\ell}^{t+1}\right) \mid x_{\ell}^{t+1}, \mathcal{F}^{t}\right] \times \operatorname{Pr}\left(k_{\ell}^{t+1}=k^{\prime}, e_{\ell}^{t+1}=0 \mid x_{\ell}^{t+1}, \mathcal{F}^{t}\right) \mid \mathcal{F}^{t}\right\} \\
& =\max _{k^{\prime},\left\{\widetilde{e}_{k}^{t+1}(.)\right\}}-C_{k k^{\prime}}+\omega_{\ell, k^{\prime}}^{t}+b_{k^{\prime}} \\
& +\delta \widehat{\phi}^{t+1} \theta_{k^{\prime}}^{t} q\left(\theta_{k^{\prime}}^{t}\right) E\left\{\mathcal{L}_{W}^{t+1}\left(k^{\prime}, 1, x_{\ell^{\prime}}^{t+1}, \omega_{\ell}^{t+1}\right) \widetilde{e}_{k^{\prime}}^{t+1}\left(x_{\ell}^{t+1}\right) \mid \mathcal{F}^{t}\right\} \\
& +\delta \widehat{\phi}^{t+1} E\left\{\left(1-\theta_{k}^{t} q\left(\theta_{k^{\prime}}^{t}\right) \widetilde{e}_{k^{\prime}}^{t+1}\left(x_{\ell}^{t+1}\right)\right) \mathcal{L}_{W}^{t+1}\left(s^{\prime}, 0, x_{\ell}^{t+1}, \omega_{\ell}^{t+1}\right) \mid \mathcal{F}^{t}\right\}
\end{aligned}
$$

For an employed worker in sector $k, k_{\ell}^{t}=k, e_{\ell}^{t}=1$ :

$$
\begin{aligned}
\mathcal{L}_{W}^{t}\left(k_{\ell}^{t}=k, e_{\ell}^{t}=1, x_{\ell}^{t}, \omega_{\ell}^{t}\right) & =\max _{\left\{\widetilde{e}_{k}^{t+1}(.)\right\}} \widetilde{\lambda}^{t} w_{k}^{t}\left(x_{\ell}^{t}\right)+\eta_{k}+\delta \widehat{\phi}^{t+1} E_{t} \mathcal{L}_{W}^{t+1}\left(k, e_{\ell}^{t+1}, x_{\ell}^{t}, \omega_{\ell}^{t+1}\right) \\
& =\max _{\left\{\tilde{e}_{k}^{t+1}(.)\right\}} \widetilde{\lambda}^{t} w_{k}^{t}\left(x_{\ell}^{t}\right)+\eta_{k} \\
& +\delta \widehat{\phi}^{t+1} E\left\{\begin{array}{c}
E\left[\mathcal{L}_{W}^{t+1}\left(k, 1, x_{\ell}^{t}, \omega_{\ell}^{t+1}\right) \mid k_{\ell}^{t+1}=k,, e_{\ell}^{t+1}=1, x_{\ell}^{t+1}, \mathcal{F}^{t}\right] \times \\
\operatorname{Pr}\left(k_{\ell}^{t+1}=k, e_{\ell}^{t+1}=1 \mid x_{\ell}^{t+1}, \mathcal{F}^{t}\right) \mid \mathcal{F}^{t}
\end{array}\right\} \\
+ & \delta \widehat{\phi}^{t+1} E\left\{\begin{array}{c}
E\left[\mathcal{L}_{W}^{t+1}\left(k, 0, x_{\ell}^{t}, \omega_{\ell}^{t+1}\right) \mid k_{\ell}^{t+1}=k, e_{\ell}^{t+1}=0, x_{\ell}^{t+1}, \mathcal{F}^{t}\right] \times \\
\operatorname{Pr}\left(k_{\ell}^{t+1}=k, e_{\ell}^{t+1}=0 \mid x_{\ell}^{t+1}, \mathcal{F}^{t}\right) \mid \mathcal{F}^{t}
\end{array}\right\} \\
= & \max _{\left\{\widetilde{e}_{k}^{t+1}(.)\right\}} \widetilde{\lambda}^{t} w_{k}^{t}\left(x_{\ell}^{t}\right)+\eta_{k} \quad \begin{array}{c}
\widetilde{e}_{k}^{t+1}\left(x_{\ell}^{t}\right) \mathcal{L}_{W}^{t+1}\left(k, 1, x_{\ell}^{t}, \omega_{\ell}^{t+1}\right) \\
+
\end{array} \\
+ & \delta \widehat{\phi}^{t+1}\left(1-\chi_{k}\right) E\left[\begin{array}{c}
\left.+1-\widetilde{e}_{k}^{t+1}\left(x_{\ell}^{t}\right)\right) \mathcal{L}_{W}^{t+1}\left(k, 0, x_{\ell}^{t}, \omega_{\ell}^{t+1}\right) \mid \mathcal{F}^{t}
\end{array}\right] \\
+ & \delta \widehat{\phi}^{t+1} \chi_{k} E\left[\mathcal{L}_{W}^{t+1}\left(k, 0, x_{\ell}^{t}, \omega_{\ell}^{t+1}\right) \mid \mathcal{F}^{t}\right]
\end{aligned}
$$

Make the following definitions

$$
\begin{gathered}
\widetilde{U}_{k}^{t}\left(\omega_{\ell}^{t}\right) \equiv \mathcal{L}_{W}^{t}\left(k_{\ell}^{t}=k, e_{\ell}^{t}=0, x_{\ell}^{t}, \omega_{\ell}^{t}\right), \text { and } \\
W_{k}^{t}(x) \equiv \mathcal{L}_{W}^{t}\left(k_{\ell}^{t}=k, e_{\ell}^{t}=1, x, \omega_{\ell}^{t}\right) .
\end{gathered}
$$

$\widetilde{U}_{k}^{t}\left(\omega_{\ell}^{t}\right)$ is the value of unemployment in sector $k$, conditional on the preference shocks $\omega_{\ell}^{t}$, and $W_{k}^{t}(x)$ is the value of a job with match productivity $x$. Note that $\mathcal{L}_{W}^{t}\left(k_{\ell}^{t}=k, e_{\ell}^{t}=0, x_{\ell}^{t}, \omega_{\ell}^{t}\right)$ does not depend on $x_{\ell}^{t}$ and $\mathcal{L}_{W}^{t}\left(k_{\ell}^{t}=k, e_{\ell}^{t}=1, x, \omega_{\ell}^{t}\right)$ does not depend on $\omega_{\ell}^{t}$. Rewrite $\widetilde{U}_{k}^{t}\left(\omega_{\ell}^{t}\right)$ as

$$
\begin{aligned}
\widetilde{U}_{k}^{t}\left(\omega_{\ell}^{t}\right) & =\max _{k^{\prime},\left\{\widetilde{e}_{k}^{t+1}(.)\right\}}-C_{k k^{\prime}}+\omega_{\ell, k^{\prime}}^{t}+b_{k^{\prime}} \\
& +\delta \widehat{\phi}^{t+1} \theta_{k^{\prime}}^{t} q\left(\theta_{k^{\prime}}^{t}\right) \int W_{k^{\prime}}^{t+1}(x) \widetilde{e}_{k^{\prime}}^{t+1}(x) d G_{k^{\prime}}(x) \\
& +\delta \widehat{\phi}^{t+1}\left(1-\theta_{k^{\prime}}^{t} q\left(\theta_{k^{\prime}}^{t}\right) \operatorname{Pr}\left(\widetilde{e}_{k^{\prime}}^{t+1}\left(x_{\ell}^{t+1}\right)=1\right)\right) E_{\omega}\left(\widetilde{U}_{k^{\prime}}^{t+1}\left(\omega_{\ell}^{t+1}\right)\right),
\end{aligned}
$$


and so:

$$
\begin{aligned}
\widetilde{U}_{k}^{t}\left(\omega_{\ell}^{t}\right) & =\max _{k^{\prime},\left\{\widetilde{e}_{k}^{t+1}(.)\right\}}-C_{k k^{\prime}}+\omega_{\ell, k^{\prime}}^{t}+b_{k^{\prime}} \\
& +\delta \widehat{\phi}^{t+1} \theta_{k^{\prime}}^{t} q\left(\theta_{k^{\prime}}^{t}\right) \int\left(\begin{array}{c}
W_{k^{\prime}}^{t+1}(x) \widetilde{e}_{k^{\prime}}^{t+1}(x)+ \\
E_{\omega}\left(\widetilde{U}_{k^{\prime}}^{t+1}\left(\omega_{\ell}^{t+1}\right)\right)\left(1-\widetilde{e}_{k^{\prime}}^{t+1}(x)\right)
\end{array}\right) d G_{k^{\prime}}(x) \\
& +\delta \widehat{\phi}^{t+1}\left(1-\theta_{k^{\prime}}^{t} q\left(\theta_{k^{\prime}}^{t}\right)\right) E_{\omega}\left(\widetilde{U}_{k^{\prime}}^{t+1}\left(\omega_{\ell}^{t+1}\right)\right) .
\end{aligned}
$$

Now, wewrite $W_{k}^{t}(x)$ as:

$$
\begin{aligned}
& W_{k}^{t}(x)=\max _{\left\{\widetilde{e}_{k}^{t+1}(.)\right\}} \widetilde{\lambda}^{t} w_{k}^{t}(x)+\eta_{k} \\
& +\delta \widehat{\phi}^{t+1}\left(1-\chi_{k}\right) \widetilde{e}_{k}^{t+1}(x) W_{k}^{t+1}(x) \\
& +\delta \widehat{\phi}^{t+1}\left(1-\left(1-\chi_{k}\right) \widetilde{e}_{k}^{t+1}(x)\right) E\left(\widetilde{U}_{k}^{t+1}\left(\omega_{\ell}^{t+1}\right)\right),
\end{aligned}
$$

and so

$$
\begin{aligned}
& W_{k}^{t}(x)=\max _{\left\{\widetilde{e}_{k}^{t+1}(.)\right\}} \widetilde{\lambda}^{t} w_{k}^{t}\left(x_{\ell}^{t}\right)+\eta_{k} \\
& +\delta \widehat{\phi}^{t+1}\left(1-\chi_{k}\right)\left(\widetilde{e}_{k}^{t+1}(x) W_{k}^{t+1}(x)+\left(1-\widetilde{e}_{k}^{t+1}(x)\right) E_{\omega}\left(\widetilde{U}_{k}^{t+1}\left(\omega_{\ell}^{t+1}\right)\right)\right) \\
& +\delta \widehat{\phi}^{t+1} \chi_{k} E_{\omega}\left(\widetilde{U}_{k}^{t+1}\left(\omega_{\ell}^{t+1}\right)\right) .
\end{aligned}
$$

It is now clear that the optimal policy $\widetilde{e}_{k}^{t+1}($.$) is:$

$$
\widetilde{e}_{k}^{t+1}(x)=\left\{\begin{array}{c}
1 \text { if } W_{k}^{t+1}(x)>E_{\omega}\left(\widetilde{U}_{k}^{t+1}\left(\omega_{\ell}^{t+1}\right)\right) \\
0 \text { otherwise }
\end{array}\right\} .
$$

Define $U_{k}^{t} \equiv E_{\omega}\left(\widetilde{U}_{k}^{t}\left(\omega_{\ell}^{t}\right)\right)$. We therefore have the following Bellman equations:

$$
\begin{gathered}
\left.U_{k}^{t}=E_{\omega}\left(\begin{array}{c}
\max _{k^{\prime}}-C_{k k^{\prime}}+b_{k^{\prime}}+\omega_{\ell, k^{\prime}}^{t} \\
+\delta \widehat{\phi}^{t+1} \theta_{k^{\prime}}^{t} q\left(\theta_{k^{\prime}}^{t}\right) \int \max \left\{W_{k^{\prime}}^{t+1}(x), U_{k^{\prime}}^{t+1}\right\} \\
+\delta \widehat{\phi}^{t+1}\left(1-\theta_{k^{\prime}}^{t} q\left(\theta_{k^{\prime}}^{t}\right)\right) U_{k^{\prime}}^{t+1}
\end{array}\right) d G_{k^{\prime}}(x)\right) \\
W_{k}^{t}(x)=\widetilde{\lambda}^{t} w_{k}^{t}(x)+\eta_{k}+\delta \widehat{\phi}^{t+1}\left(1-\chi_{k}\right)\left(\max \left\{W_{k}^{t+1}(x), U_{k}^{t+1}\right\}\right)+\delta \widehat{\phi}^{t+1} \chi_{k} U_{k}^{t+1}
\end{gathered}
$$




\section{B Steady State Equilibrium}

In this section we derive the equations characterizing the steady state equilibrium. The key conditions that we impose is that variables are constant over time, inflows of workers into each sector equal outflows, and job destruction rates equal job creation rates. We also impose that the preference shifters $\left\{\phi_{i}^{t}\right\}$ are constant and equal to 1 in the long run.

Wage Equation

$$
w_{k, i}(x)=\beta_{k, i} \widetilde{w}_{k, i} x+\frac{\left(1-\beta_{k, i}\right)(1-\delta) U_{k, i}-\left(1-\beta_{k, i}\right) \eta_{k, i}}{\widetilde{\lambda}_{i}}
$$

Firms' value function

$$
J_{k, i}(x)=\frac{1-\beta_{k, i}}{1-\left(1-\chi_{k, i}\right) \delta} \widetilde{\lambda}_{i} \widetilde{w}_{k, i}\left(x-\underline{x}_{k, i}\right)
$$

Probability of filling a vacancy

$$
q_{i}\left(\theta_{k, i}\right)=\frac{\kappa_{k, i} P_{i}^{F}}{\widetilde{w}_{k, i}} \times \frac{1-\delta\left(1-\chi_{k, i}\right)}{\delta\left(1-\beta_{k, i}\right) I_{k, i}\left(\underline{x}_{k, i}\right)}
$$

where

$$
I_{k, i}\left(\underline{x}_{k, i}\right) \equiv \int_{\underline{x}_{k, i}}^{x_{\max }}\left(s-\underline{x}_{k, i}\right) d G_{k, i}(s)
$$

\section{Unemployed workers' Bellman equation}

$$
U_{k, i}=\zeta_{i} \log \left(\sum_{k^{\prime}} \exp \left\{\frac{-C_{k k^{\prime}, i}+b_{k^{\prime}, i}+\theta_{k^{\prime}, i} \frac{\kappa_{k^{\prime}, i} P_{i}^{F}}{\widetilde{w}_{k^{\prime}, i}} \times \widetilde{\lambda}_{i} \widetilde{w}_{k^{\prime}, i} \frac{\beta_{k^{\prime}, i}}{\left(1-\beta_{k^{\prime}, i}\right)}+\delta U_{k^{\prime}, i}}{\zeta_{i}}\right\}\right)
$$

\section{Transition rates}

$$
s_{k \ell, i}=\frac{\exp \left\{\frac{-C_{k \ell, i}+b_{\ell, i}+\theta_{\ell, i} \frac{\kappa_{\ell, i} P_{i}^{F}}{\widehat{w}_{\ell, i}} \times \widetilde{\lambda}_{i} \widetilde{w}_{\ell, i} \frac{\beta_{\ell, i}}{\left(1-\beta_{\ell, i}\right)}+\delta U_{\ell, i}}{\zeta_{i}}\right\}}{\sum_{\bar{k}} \exp \left\{\frac{-C_{k \bar{k}, i}+b_{\bar{k}, i}+\theta_{\bar{k}, i} \frac{\kappa_{\bar{k}, i} P_{i}^{F}}{\widetilde{w}_{\bar{k}, i}} \times \widetilde{\lambda}_{i} \widetilde{w}_{\bar{k}, i} \frac{\beta_{\bar{k}, i}}{\left(1-\beta_{\bar{k}, i}\right)}+\delta U_{\bar{k}, i}}{\zeta_{i}}\right\}}
$$

Steady-state unemployment rates

$$
u_{k, i}=\frac{\chi_{k, i}}{\theta_{k, i} q_{i}\left(\theta_{k, i}\right)\left(1-G_{k, i}\left(\underline{x}_{k, i}\right)\right)+\chi_{k, i}}
$$


Trade + Price System

Input Bundle Price

$$
P_{k, i}^{M}=\prod_{\ell=1}^{K}\left(\frac{P_{\ell, i}^{I}}{\nu_{k \ell, i}}\right)^{\nu_{k \ell, i}}
$$

Domestic Sectoral Output Price

$$
c_{k, i}=\left(\frac{\widetilde{w}_{k, i}}{\gamma_{k, i}}\right)^{\gamma_{k, i}}\left(\frac{P_{k, i}^{M}}{1-\gamma_{k, i}}\right)^{1-\gamma_{k, i}}
$$

Price of Composite Sector-Specific Intermediate Good

$$
P_{k, i}^{I}=\Gamma_{k, i}\left[\sum_{j=1}^{N} \frac{A_{k, j}}{\left(c_{k, j} d_{k, j i}\right)^{\lambda}}\right]^{-1 / \lambda}
$$

where $\Gamma_{k, i}$ is a sector and country specific constant.

Price of Final Consumption Good

$$
P_{i}^{F}=\prod_{k=1}^{K}\left(\frac{P_{k, i}^{I}}{\mu_{k, i}}\right)^{\mu_{k i}}
$$

Trade Shares

$$
\pi_{k, o i}=\frac{A_{k, o}\left(c_{k, o} d_{k, o i}\right)^{-\lambda}}{\Phi_{k, i}}
$$

where

$$
\Phi_{k, i}=\sum_{o=1}^{N} A_{k, o}\left(c_{k, o} d_{k, o i}\right)^{-\lambda}
$$

\section{Zero net flows condition}

$$
\left(L_{i} \cdot u_{i}\right)=\left(\sum_{\ell=1}^{K} s_{\ell k, i} L_{\ell, i} u_{\ell, i}\right)_{k=1}^{K}=s_{i}^{\prime}\left(L_{i} \cdot u_{i}\right)
$$

Product market clearing

Gross Output

$$
\begin{aligned}
\gamma_{k, o} Y_{k, o} & =\widetilde{w}_{k, o} L_{k, o}\left(1-u_{k, o}\right) \int_{\underline{x}_{k, o}}^{x_{\max }} \frac{s}{1-G_{k, i}\left(\underline{x}_{k, o}\right)} d G_{k, o}(s) \\
& =\widetilde{w}_{k, o} \widetilde{L}_{k, o}
\end{aligned}
$$

Expenditure with Vacancies

$$
E_{k, o}^{V}=\kappa_{k, o} P_{o}^{F} \theta_{k, o} u_{k, o} L_{k, o}
$$


Market Clearing System

$$
\begin{gathered}
Y_{k, o}=\sum_{i=1}^{N} \pi_{k, o i} E_{k, i} \\
E_{k, i}=\mu_{k, i}\left(\sum_{\ell=1}^{K} \gamma_{\ell, i} Y_{\ell, i}\right)+\sum_{\ell=1}^{K}\left(1-\gamma_{\ell, i}\right) \nu_{\ell k, i} Y_{\ell, i}-\mu_{k, i} N X_{i}
\end{gathered}
$$

Normalization: World total revenue is the numeraire

$$
\sum_{i=1}^{N} \sum_{k=1}^{K} Y_{k, i}=1
$$

Final Good Consumption Expenditure

$$
E_{i}^{C}=\sum_{k=1}^{K} \gamma_{k, i} Y_{k, i}-\sum_{k=1}^{K} E_{k, i}^{V}-N X_{i}
$$

\section{Lagrange multipliers}

$$
\widetilde{\lambda}_{i}=\frac{\bar{L}_{i}}{E_{i}^{C}}
$$




\section{Solution Methods}

This Section presents the different algorithms we developped to estimate the model and to perform counterfactual simulations. Section C.1 details the estimation algorithm and Section C.2 obtains expressions for simulated moments. Section C.3 outlines an exact hat alebra algorithm to compute changes in the steady state equilibrium in response to shocks in trade costs, productivities or net exports. Section C.4 develops the algorithm solving for the transition path of our complete model with trade imbalances. Section C.5 adapts this algorithm to the case where we have exogenous deficits. Finally, Section C.6 outlines the procedure we use in Section 5.1 to extract the shocks in trade costs, productivities and inter-temporal shocks.

\section{C.1 Estimation Algorithm}

Define $I_{k, i}(x) \equiv \int_{x}^{x_{\max }}(s-x) d G_{k, i}(s)$. Imposing $G_{k, i} \sim \log \mathcal{N}\left(0, \sigma_{k, i}^{2}\right)$ and a bit of algebra leads to:

- $G_{k, i}(x)=\Phi\left(\frac{\ln x}{\sigma_{k, i}}\right)$

- $I_{k, i}(x)=\exp \left(\frac{\sigma_{k, i}^{2}}{2}\right) \Phi\left(\sigma_{k, i}-\frac{\ln x}{\sigma_{k, i}}\right)-x \Phi\left(-\frac{\ln x}{\sigma_{k, i}}\right)$

- $I_{k, i}(0)=\exp \left(\frac{\sigma_{k, i}^{2}}{2}\right)$

- $\int_{\underline{x}_{k, i}}^{x_{\max }} \frac{s}{1-G_{k, i}\left(\underline{x}_{k, i}\right)} d G_{k, i}(s)=\exp \left(\frac{\sigma_{k, i}^{2}}{2}\right) \frac{\Phi\left(\sigma_{k, i}-\frac{\ln \underline{x} k, i}{\sigma_{k, i}}\right)}{\Phi\left(-\frac{\ln \underline{x}_{k, i}}{\sigma_{k, i}}\right)}$

Note: The estimation procedure we describe takes trade shares $\pi_{k, o i}^{D a t a}$ and net exports $N X_{i}^{D a t a}$ as given.

Step 1: Solve for $\left\{Y_{k, i}\right\}$ using:

$$
\begin{gathered}
Y_{k, o}=\sum_{i=1}^{N} \sum_{\ell=1}^{K} \pi_{k, o i}^{D a t a}\left(\mu_{k, i} \gamma_{\ell, i}+\left(1-\gamma_{\ell, i}\right) \nu_{\ell k, i}\right) Y_{\ell, i}-\sum_{i=1}^{N} \pi_{k, o i}^{D a t a} \mu_{k, i} N X_{i} \\
\sum_{o=1}^{N} \sum_{k=1}^{K} Y_{k, o}=1
\end{gathered}
$$

The rest of the procedure conditions on these values of $\left\{Y_{k, i}\right\}$.

Step 2: Guess model parameters $\Omega$. We treat $\widetilde{\kappa}_{k, i} \equiv \frac{\kappa_{k, i} P_{i}^{F}}{\widetilde{w}_{k, i}}$ as parameters to be estimated. 
Step 3: Define

$$
\varpi_{k, i} \equiv \frac{\left(1-\left(1-\chi_{k, i}\right) \delta\right) \widetilde{\kappa}_{k, i}}{\delta\left(1-\beta_{k, i}\right)}
$$

If $\frac{\left(1-\left(1-\chi_{k, i}\right) \delta\right) \widetilde{\kappa}_{k, i}}{\delta\left(1-\beta_{k, i}\right) I_{k, i}(0)}=\frac{\varpi_{k, i}}{I_{k, i}(0)} \geq 1$, the free entry condition cannot be satisfied- $I_{k, i}$ is decreasing. Abort the procedure and highly penalize the objective function.

Step 4: Find $\underline{x}_{k, i}^{u b}$ such that $\frac{\left(1-\left(1-\chi_{k, i}\right) \delta\right) \widetilde{\kappa}_{k, i}}{\delta\left(1-\beta_{k, i}\right) I_{k, i}\left(\underline{x}_{k, i}^{u b}\right)}=1 \Longleftrightarrow I_{k, i}\left(\underline{x}_{k, i}^{u b}\right)=\varpi_{k, i}$. If along the algorithm $\underline{x}_{k, i}$ goes above $\underline{x}_{k, i}^{u b}$, we update it to be equal to $\underline{x}_{k, i}^{u b}$ (minus a small number).

Step 5: Guess $\left\{L_{k, i}\right\}$, and $\left\{\underline{x}_{k, i}\right\}$

Step 6: Compute $I_{k, i}\left(\underline{x}_{k, i}\right), G_{k, i}\left(\underline{x}_{k, i}\right), \theta_{k, i}$ and $u_{k, i}$.

- $\theta_{k, i}=q_{i}^{-1}\left(\frac{\varpi_{k, i}}{I_{k, i}\left(\underline{x}_{k, i}\right)}\right)$ where $q_{i}^{-1}(y)=\left(\frac{1-y^{\xi_{i}}}{y^{\xi_{i}}}\right)^{1 / \xi_{i}}$

- $u_{k, i}=\frac{\chi_{k, i}}{\theta_{k, i} q_{i}\left(\theta_{k, i}\right)\left(1-G_{k, i}\left(\underline{x}_{k, i}\right)\right)+\chi_{k, i}}$

Step 7: Compute $\left\{\widetilde{L}_{k, i}\right\}$

$$
\begin{aligned}
\widetilde{L}_{k, i} & \equiv L_{k, i}\left(1-u_{k, i}\right) \int_{\underline{x}_{k, i}}^{x_{\max }} \frac{s}{1-G_{k, i}\left(\underline{x}_{k, i}\right)} d G_{k, i}(s) \\
& =L_{k, i}\left(1-u_{k, i}\right) \exp \left(\frac{\sigma_{k, i}^{2}}{2}\right) \frac{\Phi\left(\sigma_{k, i}-\frac{\ln \underline{x}_{k, i}}{\sigma_{k, i}}\right)}{\Phi\left(-\frac{\ln \underline{x}_{k, i}}{\sigma_{k, i}}\right)}
\end{aligned}
$$

Step 8: Compute $\left\{\widetilde{w}_{k, i}\right\}$

$$
\widetilde{w}_{k, i}=\frac{\gamma_{k, i} Y_{k, i}}{\widetilde{L}_{k, i}}
$$

Step 9: Compute $\left\{E_{k, i}^{V}\right\}$

$$
E_{k, i}^{V}=\widetilde{\kappa}_{k, i} \widetilde{w}_{k, i} \theta_{k, i} u_{k, i} L_{k, i}
$$

Step 10: Compute $\left\{E_{i}^{C}\right\}$

$$
E_{i}^{C}=\sum_{k=1}^{K} \gamma_{k, i} Y_{k, i}-\sum_{k=1}^{K} E_{k, i}^{V}-N X_{i}
$$

Step 11: Compute $\left\{\widetilde{\lambda}_{i}\right\}$

$$
\widetilde{\lambda}_{i}=\frac{\bar{L}_{i}}{E_{i}^{C}}
$$

Step 12: Obtain $\left\{U_{k, i}\right\}$. 
- Step 12a: Guess $\left\{U_{k i}^{0}\right\}$

- Step 12b: Compute until convergence

$$
U_{k, i}^{g+1}=\zeta_{i} \log \left(\sum_{\ell=1}^{K} \exp \left\{\frac{-C_{k \ell, i}+b_{\ell, i}+\theta_{\ell, i} \widetilde{\kappa}_{\ell, i} \widetilde{\lambda}_{i} \widetilde{w}_{\ell, i} \frac{\beta_{\ell, i}}{\left(1-\beta_{\ell, i}\right)}+\delta U_{\ell, i}-\delta U_{k, i}^{g}}{\zeta_{i}}\right\}\right)+\delta U_{k, i}^{g}
$$

Step 13: Update $\left\{L_{k, i}\right\}$.

- Step 13a: Given knowledge of $\left\{U_{k, i}\right\}$, compute transition rates $s_{k \ell, i}$.

$$
s_{k \ell, i}=\frac{\exp \left\{\frac{-C_{k \ell, i}+b_{\ell, i}+\theta_{\ell, i} \widetilde{\kappa}_{\ell, i} \widetilde{\lambda}_{i} \widetilde{w}_{\ell, i} \frac{\beta_{\ell, i}}{1-\beta_{\ell, i}}+\delta U_{\ell, i}}{\zeta_{i}}\right\}}{\sum_{\bar{k}} \exp \left\{\frac{-C_{k \bar{k}, i}+b_{\bar{k}, i}+\theta_{\bar{k}, i} \widetilde{\kappa}_{\bar{k}, i} \widetilde{\lambda}_{i} \widetilde{w}_{\bar{k}, i} \frac{\beta_{\bar{k}, i}}{1-\beta_{\bar{k}, i}}+\delta U_{\bar{k}, i}}{\zeta_{i}}\right\}}
$$

- Step 13b: Find $y_{i}$ such that

$$
\left(I-s_{i}^{\prime}\right) y_{i}=0
$$

- Step 13c: Find allocations $L_{k, i}$

$$
\begin{gathered}
L_{k, i} u_{k, i}=\varphi y_{k, i} \\
\Rightarrow L_{k, i}=\varphi \underbrace{y_{k, i} / u_{k, i}}_{\widetilde{y}_{k, i}} \\
\Rightarrow L_{i}^{\prime} 1_{K \times 1}=\varphi \widetilde{y}_{k, i}^{\prime} 1_{K \times 1}=\bar{L}_{i} \\
\Rightarrow \varphi=\frac{\bar{L}_{i}}{\widetilde{y}_{k, i}^{\prime} 1_{K \times 1}} \\
\left(L_{k, i}\right)^{\prime}=\varphi \widetilde{y}_{k, i} \\
L_{k, i}^{n e w}=\left(1-\lambda_{L}\right) L_{k, i}+\lambda_{L}\left(L_{k, i}\right)^{\prime}
\end{gathered}
$$

Step 14: Update $\left\{\underline{x}_{k, i}\right\}$.

Note that in equilibrium:

$$
\widetilde{\lambda}_{i} \widetilde{w}_{k, i} \underline{x}_{k, i}=(1-\delta) U_{k, i}-\eta_{k, i}
$$

So, we update $\underline{x}_{k, i}$ according to:

$$
\begin{gathered}
\left(\underline{x}_{k, i}\right)^{\prime}=\frac{(1-\delta) U_{k, i}-\eta_{k, i}}{\widetilde{\lambda}_{i} \widetilde{w}_{k, i}} \\
\underline{x}_{k, i}^{\text {new }}=\min \left\{\left(1-\lambda_{x}\right) \underline{x}_{k, i}+\lambda_{x}\left(\underline{x}_{k, i}\right)^{\prime}, \underline{x}_{k, i}^{u b}\right\}
\end{gathered}
$$


Step 15: Armed with $L_{k, i}^{n e w}$ and $\underline{x}_{k, i}^{n e w}$ go to Step 6 until $\left\|\left\{L_{k, i}^{n e w}-L_{k, i}\right\}\right\| \rightarrow 0$ and $\left\|\left\{\underline{x}_{k, i}^{n e w}-\underline{x}_{k, i}\right\}\right\| \rightarrow$ 0 .

Note that $\left\|\left\{\underline{x}_{k, i}^{\text {new }}-\underline{x}_{k, i}\right\}\right\| \rightarrow 0$ does not imply that (A.37) is satisfied. Therefore, we penalize deviations from (A.37) in the objective function.

Step 16: Generate moments, compute Loss Function, guess new parameter set $\Omega$ and go to Step 3 , until objective function is minimized.

Note: Given that we condition on the trade shares $\pi_{k, o i}^{\text {Data }}$, we can estimate the model country by country, separately. However, in practice, we will first estimate the model for the US and obtain all of the US specific parameters. Next, armed with US-specific mobility costs $C_{k k^{\prime}}$ and sector-specific exogenous exit components $\chi_{k}$ (we will impose $\chi_{k, i}=\chi_{i}+\chi_{k}$ ), we estimate the remaining countries' parameters separately, in parallel.

\section{C.2 Expressions for Simulated Moments}

\section{C.2.1 Employment Shares}

$$
e m p_{k, i}=\frac{L_{k, i}\left(1-u_{k, i}\right)}{\sum_{k=1}^{K} L_{k, i}\left(1-u_{k, i}\right)}
$$

\section{C.2.2 National Unemployment Rate}

$$
\text { unemp }_{i}=\frac{\sum_{k=1}^{K} L_{k, i} u_{k, i}}{\sum_{k=1}^{K} L_{k, i}}
$$

\section{C.2.3 Sector-Specific Average Wages}

$$
\begin{gathered}
w_{k, i}(x)=\left(1-\beta_{k, i}\right) \widetilde{w}_{k, i} \underline{x}_{k, i}+\beta_{k, i} \widetilde{w}_{k, i} x \\
\bar{w}_{k, i}=\frac{\int_{\underline{x}_{k, i}}^{x_{\max }} w_{k, i}(s) d G_{k, i}(s)}{1-G_{k, i}\left(\underline{x}_{k, i}\right)} \\
=\left(1-\beta_{k, i}\right) \widetilde{w}_{k, i} \underline{x}_{k, i}+\beta_{k, i} \widetilde{w}_{k, i} \int_{\underline{x}_{k, i}}^{x_{\max }} \frac{s}{1-G_{k, i}\left(\underline{x}_{k, i}\right)} d G_{k, i}(s) \\
=\left(1-\beta_{k, i}\right) \widetilde{w}_{k, i} \underline{x}_{k, i}+\beta_{k, i} \widetilde{w}_{k, i} \exp \left(\frac{\sigma_{k, i}^{2}}{2}\right) \frac{\Phi\left(\sigma_{k, i}-\frac{\ln \underline{x}_{k, i}}{\sigma_{k, i}}\right)}{\Phi\left(-\frac{\ln \underline{x}_{k, i}}{\sigma_{k, i}}\right)}
\end{gathered}
$$




\section{C.2.4 Sector-Specific Variance of Wages}

$$
\begin{aligned}
\sigma_{w, k, i}^{2} & =\frac{\int_{\underline{x}_{k, i}}^{\infty}\left(w_{k, i}(s)-\bar{w}_{k, i}\right)^{2} d G_{k, i}(s)}{1-G_{k, i}\left(\underline{x}_{k, i}\right)} \\
& =\left(\beta_{k, i} \widetilde{w}_{k, i}\right)^{2} \times \frac{\int_{\underline{x}_{k, i}}^{\infty}\left(s-\exp \left(\frac{\sigma_{k, i}^{2}}{2}\right) \frac{\Phi\left(\sigma_{k, i}-\frac{\ln \underline{x}_{k, i}}{\sigma_{k, i}}\right)}{\Phi\left(-\frac{\ln \underline{x}_{k, i}}{\sigma_{k, i}}\right)}\right)^{2} d G_{k, i}(s)}{1-G_{k, i}\left(\underline{x}_{k, i}\right)} \\
& =\left(\beta_{k, i} \widetilde{w}_{k, i}\right)^{2} \times\left(\exp \left(2 \sigma_{k, i}^{2}\right) \frac{\Phi\left(2 \sigma_{k, i}-\frac{\ln \underline{x}_{k, i}}{\sigma_{k, i}}\right)}{\Phi\left(-\frac{\ln \underline{x}_{k, i}}{\sigma_{k, i}}\right)}-\exp \left(\sigma_{k, i}^{2}\right)\left(\frac{\Phi\left(\sigma_{k, i}-\frac{\ln \underline{x}_{k, i}}{\sigma_{k, i}}\right)}{\Phi\left(-\frac{\ln \underline{x}_{k, i}}{\sigma_{k, i}}\right)}\right)^{2}\right)
\end{aligned}
$$

\section{C.2.5 Transition Rates}

Note that the transition rates in equation (10) are transitions from unemployment in sector $k$ to search in sector $k^{\prime}$ within period $t$. There are no data counterfactuals for this variable. However, we can construct a matrix with transition rates between all possible (model) states between time $t$ and time $t+N$ (where $N$ is even) - where variables are measured at the $t_{a}$ stage (which is the production stage). From this matrix, we can obtain $N$-period transition rates between all states observed in the data (employment in each of the sectors and unconditional unemployment). First, we obtain the one-year transition matrix $\widetilde{s}^{t, t+1}$ between states $\left\{\widetilde{u}_{1}, \ldots, \widetilde{u}_{K}, 1, \ldots, K\right\}$. Here, we abuse notation to mean $\widetilde{u}_{k}$ as sector- $k$ unemployment at the very beginning of a period.

The one-year transition rate between sector- $\ell$ unemployment and sector- $k$ unemployment is given by:

$$
\widetilde{s}_{\widetilde{u}_{\ell} \widetilde{u}_{k}, i}^{t, t+1}=s_{\ell k, i}^{t, t+1}\left(1-\theta_{k, i}^{t} q_{i}\left(\theta_{k, i}^{t}\right)\left(1-G_{k, i}\left(\underline{x}_{k, i}^{t+1}\right)\right)\right),
$$

that is, a share $s_{\ell k, i}^{t}$ of individuals starting period $t$ unemployed in sector $\ell$ choose to search in sector $k$. A fraction $\left(1-\theta_{k, i}^{t} q_{i}\left(\theta_{k, i}^{t}\right)\left(1-G_{k, i}\left(\underline{x}_{k, i}^{t+1}\right)\right)\right)$ of those do not find a match that survives until $t+1$. Similarly, the one-year transition rate between sector- $\ell$ unemployment and sector- $k$ employment is given by:

$$
\begin{aligned}
\widetilde{s}_{\widetilde{u}_{\ell} k, i}^{t, t+1} & =s_{\ell k, i}^{t, t+1} \theta_{k, i}^{t} q_{i}\left(\theta_{k, i}^{t}\right)\left(1-G_{k, i}\left(\underline{x}_{k, i}^{t+1}\right)\right) \\
& =s_{\ell k, i}^{t, t+1}-\widetilde{s}_{\widetilde{u}_{\ell}, t+\widetilde{u}_{k}, i} .
\end{aligned}
$$

According to the timing assumptions of the model, the one-year transition rate between employment in sector $k$ and employment in sector $k^{\prime}$ is zero if $k \neq k^{\prime}$. However, the persistence rate of employment in sector $k$ is given by the probability that a match does not receive a death shock times the probability that the match is not dissolved because the threshold for production increases in the following period:

$$
\widetilde{s}_{k k^{\prime}, i}^{t, t+1}=\left\{\begin{array}{c}
0 \text { if } k \neq k^{\prime} \\
\left(1-\chi_{k, i}\right) \operatorname{Pr}\left(x \geq \underline{x}_{k, i}^{t+1} \mid x \geq \underline{x}_{k, i}^{t}\right) \text { if } k=k^{\prime} .
\end{array}\right.
$$

Finally, the one-year transition rate between sector- $k$ employment and unemployment in sector $\ell$ 
is given by:

$$
\widetilde{s}_{k \widetilde{u}_{\ell}, i}^{t, t+1}=\left\{\begin{array}{c}
0 \text { if } k \neq \ell \\
\chi_{k, i}+\left(1-\chi_{k, i}\right) \operatorname{Pr}\left(x<\underline{x}_{k, i}^{t+1} \mid x \geq \underline{x}_{k, i}^{t}\right) \text { if } k=\ell
\end{array} .\right.
$$

That is, if a worker is employed in sector $k$ at $t$, she cannot start next period unemployed in sector $\ell$ if $k \neq \ell$. Otherwise, workers transition between sector $k$ employment to sector $k$ unemployment if their match is hit with a death shock or if their employer's productivity goes below the threshold for production at $t+1$.

We can now write the $N$-period transition matrix as:

$$
\widetilde{s}^{t, t+N}=\widetilde{s}^{t+k-1, t+k} \times \ldots \times \widetilde{s}^{t+1, t+2} \times \widetilde{s}^{t, t+1},
$$

and we can write transition rates between unemployment $\widetilde{u}$ and sector- $k$ employment between $t$ and $t+N$ as:

$$
\widetilde{s}_{\widetilde{u}, k, i}^{t, t+N}=\frac{\sum_{\ell=1}^{K} L_{\ell, i}^{t-1} \widetilde{u}_{\ell, i}^{t-1} \widetilde{s}_{\widetilde{u}_{\ell}, k}^{t, t+N}}{\sum_{\ell=1}^{K} L_{\ell, i}^{t-1} \widetilde{u}_{\ell, i}^{t-1}} .
$$

Finally, we can write transition rates between sector- $k$ employment and unemployment $\widetilde{u}$ as:

$$
\widetilde{s}_{k, \widetilde{u}, i}^{t, t+N}=1-\sum_{k^{\prime}=1}^{K} \widetilde{s}_{k, k^{\prime}, i}^{t, t+N}
$$

\section{1-period transition rates}

$$
\begin{gathered}
\widetilde{s}_{\widetilde{u}_{\ell} \widetilde{u}_{k}, i}=s_{\ell k, i}\left(1-\theta_{k, i} q_{i}\left(\theta_{k, i}\right)\left(1-G_{k, i}\left(\underline{x}_{k, i}\right)\right)\right) \\
\widetilde{s}_{\widetilde{u}_{\ell} k, i}=s_{\ell k, i} \theta_{k, i} q_{i}\left(\theta_{k, i}\right)\left(1-G_{k, i}\left(\underline{x}_{k, i}\right)\right) \\
\widetilde{s}_{\ell k, i}=\left\{\begin{array}{c}
0 \text { if } \ell \neq k \\
\left(1-\chi_{\ell, i}\right) \text { if } \ell=k
\end{array}\right. \\
\widetilde{s}_{\ell \widetilde{u}_{k}, i}=\left\{\begin{array}{c}
0 \text { if } \ell \neq k \\
\chi_{k, i} \text { if } \ell=k
\end{array}\right.
\end{gathered}
$$

\section{$N$-period transition rates from and to unconditional unemployment: $\widetilde{s}^{N}$}

$$
\begin{gathered}
\widetilde{s}_{\widetilde{u}, k, i}^{N}=\frac{\sum_{\ell=1}^{K} L_{\ell, i} u_{\ell, i} \widetilde{s}_{\widetilde{u}_{\ell}, k, i}^{N}}{\sum_{\ell=1}^{K} L_{\ell, i} u_{\ell, i}} \\
\widetilde{s}_{k, \widetilde{u}, i}^{N}=1-\sum_{\ell=1}^{K} \widetilde{s}_{k, \ell, i}^{N} .
\end{gathered}
$$




\section{C.3 Algorithm: Steady-State Equilibrium Following Shock}

Consider shocks $\left\{A_{k, i}^{0}\right\} \rightarrow\left\{A_{k, i}^{1}\right\},\left\{d_{k, o i}^{0}\right\} \rightarrow\left\{d_{k, o i}^{1}\right\},\left\{N X^{0}\right\} \rightarrow\left\{N X_{i}^{1}\right\}$

We will be using 0 superscripts to denote the initial steady state, and 1 superscripts to denote the final steady state.

Start from estimated Steady State: $\left\{L_{k, i}^{0}\right\},\left\{\underline{x}_{k, i}^{0}\right\},\left\{\widetilde{w}_{k, i}^{0}\right\},\left\{\pi_{k, o i}^{0}\right\}$

Note that $\pi_{k, o i}^{0}=\pi_{k, o i}^{D a t a}$

We also have $\widetilde{\kappa}_{k, i}^{0}=\frac{\kappa_{k, i} P_{i}^{F, 0}}{\widetilde{w}_{k, i}^{0}}$, but we do not know $\left\{P_{i}^{F, 0}\right\}$

Denote relative changes in variable $a$ by $\widehat{a}=\frac{a^{1}}{a^{0}}$

Step 1: Guess $\left\{L_{k, i}^{1}\right\}$ and $\left\{\underline{x}_{k, i}^{1}\right\}$

Step 2: Guess $\left\{\widetilde{w}_{k, i}^{1}\right\}$

- Step 2a: Compute $\widehat{\widetilde{w}}_{k, i}=\frac{\widetilde{w}_{k, i}^{1}}{\widetilde{w}_{k, i}^{0}}$ and iteratively solve for $\widehat{P}_{k, i}^{I}$ and $\widehat{c}_{k, i}$ using the system

$$
\begin{gathered}
\widehat{c}_{k, i}=\left(\widehat{\widetilde{w}}_{k, i}\right)^{\gamma_{k, i}} \prod_{\ell=1}^{K}\left(\widehat{P}_{\ell, i}^{I}\right)\left(1-\gamma_{k, i}\right) \nu_{k \ell, i} \\
\widehat{P}_{k, i}^{I}=\left(\sum_{o=1}^{N} \pi_{k, o i}^{0} \widehat{A}_{k, o}\left(\widehat{c}_{k, o} \widehat{d}_{k, o i}\right)^{-\lambda}\right)^{-1 / \lambda}
\end{gathered}
$$

- Step 2b: Compute $\widehat{P}_{k, i}^{F}$ :

$$
\widehat{P}_{i}^{F}=\prod_{k=1}^{K}\left(\widehat{P}_{k, i}^{I}\right)^{\mu_{k i}}
$$

- Step 2c: Compute $\widehat{\pi}_{k, o i}$ :

$$
\widehat{\pi}_{k, o i}=\widehat{A}_{k, o}\left(\frac{\widehat{c}_{k, o} \widehat{d}_{k, o i}}{\widehat{P}_{k, i}^{I}}\right)^{-\lambda}
$$

- Step 2d: Compute 


$$
\begin{aligned}
& -\pi_{k, o i}^{1}=\pi_{k, o i}^{0} \widehat{\pi}_{k, o i} \\
& -\widetilde{\kappa}_{k, i}^{1} \equiv \frac{\kappa_{k, i} P_{i}^{F, 1}}{\widetilde{w}_{k, i}^{1}}=\frac{\kappa_{k, i} P_{i}^{F, 0}}{\widetilde{w}_{k, i}^{0}} \frac{P_{i}^{F, 1}}{P_{i}^{F, 0}} \widetilde{w}_{k, i}^{0}=\widetilde{\kappa}_{k, i}^{0} \frac{\widehat{P}_{i}^{F}}{\widetilde{\widehat{w}}_{k, i}}
\end{aligned}
$$

Step 3: If $\widetilde{\kappa}_{k, i}^{1} \times \frac{1-\delta\left(1-\chi_{k, i}\right)}{\delta\left(1-\beta_{k, i}\right) I_{k, i}\left(\underline{x}_{k, i}^{1}\right)} \geq 1$ abort, set $\underline{x}_{k, i}^{1}$ such that $\widetilde{\kappa}_{k, i}^{1} \times \frac{1-\delta\left(1-\chi_{k, i}\right)}{\delta\left(1-\beta_{k, i}\right) I_{k, i}\left(\underline{x}_{k, i}^{1}\right)}=1-\varepsilon$ and go back to Step 1 with this new guess. If $\widetilde{\kappa}_{k, i}^{1} \times \frac{1-\delta\left(1-\chi_{k, i}\right)}{\delta\left(1-\beta_{k, i}\right) I_{k, i}\left(\underline{x}_{k, i}^{1}\right)}<1$, proceed to Step 4 .

Step 4: Compute

$$
\begin{gathered}
q_{i}\left(\theta_{k, i}^{1}\right)=\widetilde{\kappa}_{k, i}^{1} \times \frac{1-\delta\left(1-\chi_{k, i}\right)}{\delta\left(1-\beta_{k, i}\right) I_{k, i}\left(\underline{x}_{k, i}^{1}\right)} \\
\theta_{k, i}^{1}=q_{i}^{-1}\left(\widetilde{\kappa}_{k, i}^{1} \times \frac{1-\delta\left(1-\chi_{k, i}\right)}{\delta\left(1-\beta_{k, i}\right) I_{k, i}\left(\underline{x}_{k, i}^{1}\right)}\right) \\
u_{k, i}^{1}=\frac{\chi_{k, i}}{\theta_{k, i}^{1} q_{i}\left(\theta_{k, i}^{1}\right)\left(1-G_{k, i}\left(\underline{x}_{k, i}^{1}\right)\right)+\chi_{k, i}}
\end{gathered}
$$

Step 5: Solve system in $\left\{Y_{k, o}^{1}\right\}$

$$
\begin{gathered}
Y_{k, o}^{1}=\sum_{i=1}^{N} \pi_{k, o i}^{1}\left(\mu_{k, i}\left(\sum_{\ell=1}^{K} \gamma_{\ell, i} Y_{\ell, i}^{1}\right)+\sum_{\ell=1}^{K}\left(1-\gamma_{\ell, i}\right) \nu_{\ell k, i} Y_{\ell, i}^{1}\right)-\sum_{i=1}^{N} \pi_{k, o i}^{1} \mu_{k, i} N X_{i}^{1} \\
\sum_{i=1}^{N} \sum_{k=1}^{K} Y_{k, i}^{1}=1
\end{gathered}
$$

Step 6: Compute $\left\{\widetilde{L}_{k, i}^{1}\right\}$

$$
\begin{aligned}
\widetilde{L}_{k, i}^{1} & \equiv L_{k, i}^{1}\left(1-u_{k, i}^{1}\right) \int_{\underline{x}_{k, i}^{1}}^{x_{\max }} \frac{s}{1-G_{k, i}\left(\underline{x}_{k, i}^{1}\right)} d G_{k, i}(s) \\
& =L_{k, i}^{1}\left(1-u_{k, i}^{1}\right) \exp \left(\frac{\sigma_{k, i}^{2}}{2}\right) \frac{\Phi\left(\sigma_{k, i}-\frac{\ln \underline{x}_{k, i}^{1}}{\sigma_{k, i}}\right)}{\Phi\left(-\frac{\ln \underline{x}_{k, i}^{1}}{\sigma_{k, i}}\right)}
\end{aligned}
$$

Step 7: Update $\left\{\widetilde{w}_{k, i}^{1}\right\}$

$$
\left(\widetilde{w}_{k, i}^{1}\right)^{n e w}=\frac{\gamma_{k, i} Y_{k, i}^{1}}{\widetilde{L}_{k, i}^{1}}
$$

Go back to Step 2a and repeat until converegence of $\left\{\widetilde{w}_{k, i}^{1}\right\}$. 
Step 8: Compute

$$
\begin{gathered}
E_{k, i}^{V, 1}=\widetilde{\kappa}_{k, i}^{1} \widetilde{w}_{k, i}^{1} \theta_{k, i}^{1} u_{k, i}^{1} L_{k, i}^{1} \\
E_{i}^{C, 1}=\sum_{k=1}^{K} \gamma_{k, i} Y_{k, i}^{1}-\sum_{k=1}^{K} E_{k, i}^{V, 1}-N X_{i}^{1}
\end{gathered}
$$

Step 9: Obtain Lagrange Multipliers

$$
\widetilde{\lambda}_{i}^{1}=\frac{\bar{L}_{i}}{E_{i}^{C, 1}}
$$

Step 10: Compute Bellman Equations

$$
U_{k, i}^{1}=\zeta_{i} \log \left(\sum_{k^{\prime}} \exp \left\{\frac{-C_{k k^{\prime}, i}+b_{k^{\prime}, i}+\theta_{k^{\prime}, i}^{1} \widetilde{\kappa}_{k^{\prime}, i}^{1} \widetilde{\lambda}_{i}^{1} \widetilde{w}_{k^{\prime}, i}^{1} \frac{\beta_{k^{\prime}, i}}{\left(1-\beta_{k^{\prime}, i}\right)}+\delta U_{k^{\prime}, i}^{1}}{\zeta_{i}}\right\}\right)
$$

Step 11: Update $\left\{L_{k, i}^{1}\right\}$.

- Step 11a: Given knowledge of $\left\{U_{k, i}^{1}\right\}$, compute transition rates $s_{k \ell, i}^{1}$.

$$
s_{k \ell, i}^{1}=\frac{\exp \left\{\frac{-C_{k \ell, i}+b_{\ell, i}+\theta_{\ell, i}^{1} \widetilde{\kappa}_{\ell, i}^{1} \widetilde{\lambda}_{i}^{1} \widetilde{w}_{\ell, i}^{1} \frac{\beta_{\ell, i}}{\left(1-\beta_{\ell, i}\right)}+\delta U_{\ell, i}^{1}}{\zeta_{i}}\right\}}{\sum_{\bar{k}} \exp \left\{\frac{-C_{k \bar{k}, i}+b_{\bar{k}, i}+\theta_{\bar{k}, i}^{1} \widetilde{\kappa}_{\bar{k}, i}^{1} \widetilde{\lambda}_{i}^{1} \widetilde{w}_{\bar{k}, i}^{1} \frac{\beta_{\bar{k}, i}}{\left(1-\beta_{\bar{k}, i}\right)}+\delta U_{\bar{k}, i}^{1}}{\zeta_{i}}\right\}}
$$

- Step 11b: Find $y_{i}$ such that

$$
\left(I-\left(s_{i}^{1}\right)^{T}\right) y_{i}=0
$$

- Step 11c: Find allocations $L_{k, i}$

$$
\begin{gathered}
L_{k, i}^{1} u_{k, i}^{1}=\varphi y_{k, i} \\
\Rightarrow L_{k, i}^{1}=\varphi \underbrace{y_{k, i} / u_{k, i}^{1}}_{\widetilde{y}_{k, i}} \\
\Rightarrow\left(L_{i}^{1}\right)^{T} 1_{K \times 1}=\varphi \widetilde{y}_{k, i}^{T} 1_{K \times 1}=\bar{L}_{i} \\
\Rightarrow \varphi=\frac{\bar{L}_{i}}{\widetilde{y}_{k, i}^{T} 1_{K \times 1}}
\end{gathered}
$$




$$
\begin{gathered}
\left(L_{k, i}^{1}\right)^{\prime}=\varphi \widetilde{y}_{k, i} \\
\left(L_{k, i}^{1}\right)^{\text {new }}=\left(1-\lambda_{L}\right) L_{k, i}^{1}+\lambda_{L}\left(L_{k, i}^{1}\right)^{\prime}
\end{gathered}
$$

Step 12: Update $\left\{\underline{x}_{k, i}^{1}\right\}$.

Note that in equilibrium:

$$
\widetilde{\lambda}_{i}^{1} \widetilde{w}_{k, i}^{1} \underline{x}_{k, i}^{1}=(1-\delta) U_{k, i}^{1}-\eta_{k, i}
$$

So, we update $\underline{x}_{k, i}^{1}$ according to:

$$
\begin{gathered}
\left(\underline{x}_{k, i}^{1}\right)^{\prime}=\frac{(1-\delta) U_{k, i}^{1}-\eta_{k, i}}{\widetilde{\lambda}_{i}^{1} \widetilde{w}_{k, i}^{1}} \\
\left(\underline{x}_{k, i}^{1}\right)^{\text {new }}=\min \left\{\left(1-\lambda_{x}\right) \underline{x}_{k, i}^{1}+\lambda_{x}\left(\underline{x}_{k, i}^{1}\right)^{\prime}, \underline{x}_{k, i}^{u b}\right\}
\end{gathered}
$$

Step 13: Armed with $\left(L_{k, i}^{1}\right)^{\text {new }}$ and $\left(\underline{x}_{k, i}^{1}\right)^{\text {new }}$ go to Step 2 until $\left\|\left\{\left(L_{k, i}^{1}\right)^{\text {new }}-L_{k, i}^{1}\right\}\right\| \rightarrow 0$ and $\left\|\left\{\left(\underline{x}_{k, i}^{1}\right)^{\text {new }}-\underline{x}_{k, i}^{1}\right\}\right\| \rightarrow 0$. 


\section{C.4 Algorithm: Out-of-Steady-State Transition}

Inner Loop: conditional on paths for expenditures $\left\{E_{i}^{C, t}\right\}$-determined in the Outer Loop below.

Consider paths $\left\{A_{k, i}^{t}\right\}_{t=0}^{T_{S S}}$ and $\left\{d_{o, i, k}^{t}\right\}_{t=0}^{T_{S S}}$ with $A_{k, i}^{0}=1$ and $d_{o, i, k}^{0}=1$. Also, consider paths $\left\{\phi_{i}^{t}\right\}_{t=0}^{T_{S S}}$ with $\phi_{i}^{0}=1$ and $\widehat{\phi}_{i}^{t}=1$ for $T \leq t \leq T_{S S}$, for some $T<<T_{S S}$.

Step 0: Given paths $\left\{E_{i}^{C, t}\right\}$, compute paths $\left\{\widetilde{\lambda}_{i}^{t}\right\}: \widetilde{\lambda}_{i}^{t}=\frac{\bar{L}_{i}}{E_{i}^{C, t}}$

Step 1: Guess paths $\left\{\widetilde{w}_{k, i}^{t}\right\}_{t=1}^{T_{S S}}$ for each sector $k$ and country $i$.

Step 2: Compute $\underline{x}_{k, i}^{T_{S S}}$ consistent with $\widetilde{w}_{k, i}^{T_{S S}}$ and $\widetilde{\lambda}_{i}^{T_{S S}}$. Obtain $\theta_{k, i}^{T_{S S}}, U_{k, i}^{T_{S S}}, s_{k \ell, i}^{T_{S S}, T_{S S}+1}$ and $\pi_{k, o i}^{T_{S S}}$.

- Step 2a: Compute $\widehat{\widetilde{w}}_{k, i}=\frac{\widetilde{w}_{k, i}^{T_{S S}}}{\widetilde{w}_{k, i}^{0}}, \widehat{A}_{k, i}=\frac{A_{k, i}^{T_{S S}}}{A_{k, i}^{0}}$ and $\widehat{d}_{k, i}=\frac{d_{o, i, k}^{T_{S S}}}{d_{o, i, k}^{0}}$. Iteratively solve for $\widehat{P}_{k, i}^{I}$ and $\widehat{c}_{k, i}$ using the system

$$
\begin{gathered}
\widehat{c}_{k, i}=\left(\widehat{\widetilde{w}}_{k, i}\right)^{\gamma_{k, i}} \prod_{\ell=1}^{K}\left(\widehat{P}_{\ell, i}^{I}\right)^{\left(1-\gamma_{k, i}\right) \nu_{k \ell, i}} \\
\widehat{P}_{k, i}^{I}=\left(\sum_{o=1}^{N} \pi_{k, o i}^{0} \widehat{A}_{k, o}\left(\widehat{c}_{k, o} \widehat{d}_{k, o i}\right)^{-\lambda}\right)^{-1 / \lambda}
\end{gathered}
$$

- Step 2b: Compute $\widehat{P}_{k, i}^{F}$ :

$$
\widehat{P}_{i}^{F}=\prod_{k=1}^{K}\left(\widehat{P}_{k, i}^{I}\right)^{\mu_{k i}}
$$

- Step 2c: Compute

$$
\widehat{\pi}_{k, o i}=\widehat{A}_{k, o}\left(\frac{\widehat{c}_{k, o} \widehat{d}_{k, o i}}{\widehat{P}_{k, i}^{I}}\right)^{-\lambda}
$$

And obtain $\pi_{k, o i}^{T_{S S}}=\pi_{k, o i}^{0} \widehat{\pi}_{k, o i}$

- Step 2d: Compute

$$
-\widetilde{\kappa}_{k, i}^{T_{S S}}=\widetilde{\kappa}_{k, i}^{0} \frac{\widehat{P}_{i}^{F}}{\widehat{\widehat{w}}_{k, i}}
$$

- Step 2e: Guess $\left\{\underline{x}_{k, i}^{T_{S S}}\right\}$

- Step 2f: Compute

$$
\theta_{k, i}^{T_{S S}}=q_{i}^{-1}\left(\widetilde{\kappa}_{k, i}^{T_{S S}} \times \frac{1-\delta\left(1-\chi_{k, i}\right)}{\delta\left(1-\beta_{k, i}\right) I_{k, i}\left(\underline{x}_{k, i}^{T_{S S}}\right)}\right)
$$


- Step 2g: Compute Bellman Equations

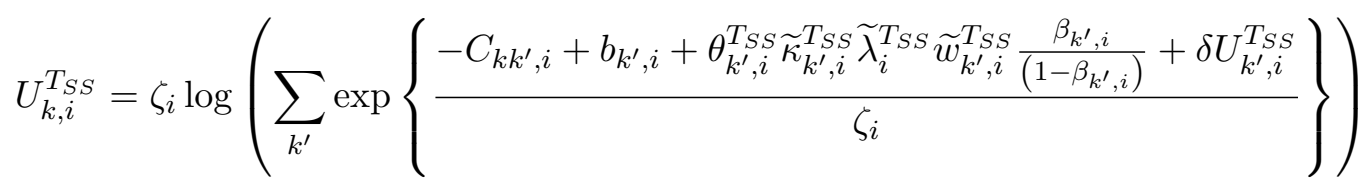

- Step 2h: Compute

$$
\left(\underline{x}_{k, i}^{T_{S S}}\right)^{\prime}=\frac{(1-\delta) U_{k, i}^{T_{S S}}-\eta_{k, i}}{\widetilde{\lambda}_{i}^{T_{S S}} \widetilde{w}_{k, i}^{T_{S S}}}
$$

- Step 2i: Update $\underline{x}_{k, i}^{T_{S S}}=\left(1-\lambda_{x}\right) \underline{x}_{k, i}^{T_{S S}}+\lambda_{x}\left(\underline{x}_{k, i}^{T_{S S}}\right)^{\prime}$, for a small step size $\lambda_{x}$, and go back to Step 2d until convergence.

- Step 2j: Compute $s_{k k^{\prime}}^{T_{S S}, T_{S S}+1}$

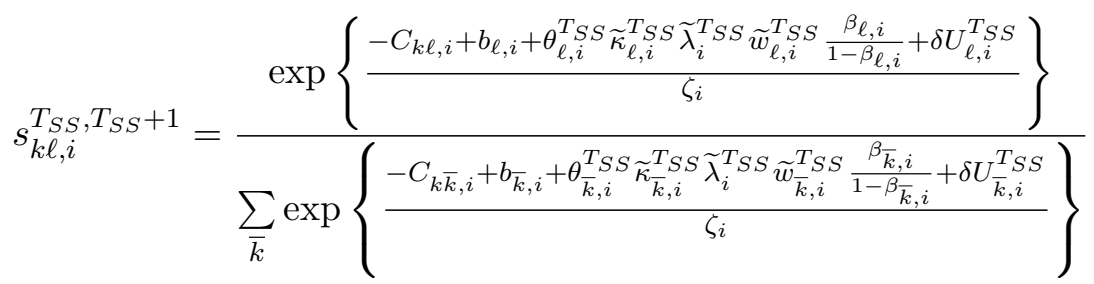

Step 3: Obtain series $\left\{\pi_{k, o i}^{t}\right\}_{t=0}^{T_{S S}},\left\{\widetilde{\kappa}_{k, i}^{t}\right\}_{t=0}^{T_{S S}}$. Define $\widehat{x}^{t} \equiv \frac{x^{t}}{x^{0}}$.

- Step 3a: For $t=1, \ldots, T_{S S}-1$ compute $\widehat{\widetilde{w}}_{k, i}^{t}=\frac{\widetilde{w}_{k, i}^{t}}{\widetilde{w}_{k, i}^{0}}$ and iteratively solve for $\widehat{P}_{k, i}^{I, t}$ and $\widehat{c}_{k, i}^{t}$ using the system

$$
\begin{aligned}
\widehat{c}_{k, i}^{t} & =\left(\widehat{\widetilde{w}}_{k, i}^{t}\right)^{\gamma_{k, i}} \prod_{\ell=1}^{K}\left(\widehat{P}_{\ell, i}^{I, t}\right)^{\left(1-\gamma_{k, i}\right) \nu_{k \ell, i}} \\
\widehat{P}_{k, i}^{I, t} & =\left(\sum_{o=1}^{N} \pi_{k, o i}^{0} \widehat{A}_{k, o}^{t}\left(\widehat{c}_{k, o}^{t} \widehat{d}_{k, o i}^{t}\right)^{-\lambda}\right)^{-1 / \lambda}
\end{aligned}
$$

- Step 3b: Compute $\widehat{P}_{k, i}^{F, t}$ for $t=1, \ldots, T_{S S}-1$ :

$$
\widehat{P}_{i}^{F, t}=\prod_{k=1}^{K}\left(\widehat{P}_{k, i}^{I, t}\right)^{\mu_{k i}}
$$

- Step 3c: Compute $\widehat{\pi}_{k, o i}^{t}$ for $t=1, \ldots, T_{S S}-1$ :

$$
\widehat{\pi}_{k, o i}^{t}=\widehat{A}_{k, o}^{t}\left(\frac{\widehat{c}_{k, o}^{t} \widehat{d}_{k, o i}^{t}}{\widehat{P}_{k, i}^{I, t}}\right)^{-\lambda}
$$

- Step 3d: Compute or $t=1, \ldots, T_{S S}-1$ : 


$$
\begin{aligned}
& -\pi_{k, o i}^{t}=\pi_{k, o i}^{0} \widehat{\pi}_{k, o i}^{t} \\
& -\widetilde{\kappa}_{k, i}^{t} \equiv \frac{\kappa_{k, i} P_{i}^{F, t}}{\widetilde{w}_{k, i}^{t}}=\frac{\kappa_{k, i} P_{i}^{F, 0}}{\widetilde{w}_{k, i}^{0}} \frac{P_{i}^{F, t}}{P_{i}^{F, 0}} \frac{\widetilde{w}_{k, i}^{0}}{\widetilde{w}_{k, i}^{t}}=\widetilde{\kappa}_{k, i}^{0} \frac{\widehat{P}_{i}^{F, t}}{\widehat{\widetilde{w}}_{k, i}^{t}}
\end{aligned}
$$

Step 4: Given knowledge of $\widetilde{w}_{k, i}^{T_{S S}}, \widetilde{\lambda}_{i}^{T_{S S}}$ and $\underline{x}_{k, i}^{T_{S S}}$ (and therefore $J_{k, i}^{T_{S S}}(s)$ ), start at $t=T_{S S}-1$ and sequentially compute (backwards) for each $t=T_{S S}-1, \ldots, 1$

- Step 4a: Given $\widetilde{w}_{k, i}^{t}, \underline{x}_{k, i}^{t+1}, \widetilde{\kappa}_{k, i}^{t}, \widetilde{\lambda}_{i}^{t}$ and $J_{k, i}^{t+1}(s)$ compute $\theta_{k, i}^{t}$.

If $\frac{\widetilde{\lambda}_{i}^{t} \widetilde{\kappa}_{k, i}^{t} \widetilde{w}_{k, i}^{t}}{\delta \widehat{\phi}_{i}^{t+1} \int_{\underline{x}_{k, i}^{t+1}}^{x+1} J_{k, i}^{t+1}(s) d G_{k, i}(s)} \leq 1$ then

$$
\theta_{k, i}^{t}=q_{i}^{-1}\left(\frac{\widetilde{\lambda}_{i}^{t} \widetilde{\kappa}_{k, i}^{t} \widetilde{w}_{k, i}^{t}}{\delta \widehat{\phi}_{i}^{t+1} \int_{\underline{x}_{k, i}^{t+1}}^{x_{\max }} J_{k, i}^{t+1}(s) d G_{k, i}(s)}\right)
$$

If $\frac{\widetilde{\lambda}_{i}^{t} \widetilde{\kappa}_{k, i}^{t} \widetilde{w}_{k, i}^{t}}{\delta \widehat{\phi}_{i}^{t+1} \int_{\underline{x}_{k, i}^{t+1}}^{x+1} J_{k, i}^{t+1}(s) d G_{k, i}(s)}>1$, it is not possible to satisfy $V_{k, i}^{t}=0$, so that $V_{k, i}^{t}<0$ and

$$
\theta_{k, i}^{t}=0 .
$$

- Step 4b: Given $\underline{x}_{k, i}^{t+1}, W_{k, i}^{t+1}(x)=\frac{\beta_{k, i}}{1-\beta_{k, i}} J_{k, i}^{t+1}(x)+U_{k, i}^{t+1}$ (for $\left.x \geq \underline{x}_{k, i}^{t+1}\right), \theta_{k, i}^{t}, U_{k, i}^{t+1}$ compute $U_{k, i}^{t}$. Notice that $\int_{\underline{x}_{k, i}^{t+1}}^{x_{\max }} W_{k, i}^{t+1}(s) d G_{k, i}(s)=\frac{\beta_{k, i}}{1-\beta_{k, i}} \int_{\underline{x}_{k, i}^{t+1}}^{x_{\max }} J_{k, i}^{t+1}(s) d G_{k, i}(s)+\left(1-G_{k, i}\left(\underline{x}_{k, i}^{t+1}\right)\right) U_{k, i}^{t+1}$ so that:

$$
U_{k, i}^{t}=\zeta_{i} \log \left(\sum_{k^{\prime}} \exp \left\{\frac{+\delta \widehat{\phi}_{i}^{t+1} \theta_{k^{\prime}, i}^{t} q_{i}\left(\theta_{k^{\prime}, i}^{t}\right) \frac{\beta_{k, i}}{1-\beta_{k, i}} \int_{\underline{x}_{k, i}^{t+1}}^{x_{\text {max }}} J_{k, i}^{t+1}(s) d G_{k, i}(s)+\delta \widehat{\phi}_{i}^{t+1} U_{k^{\prime}, i}^{t+1}}{\zeta_{i}}\right\}\right)
$$

- Step 4c: Given $J_{k, i}^{t+1}(x), \widetilde{w}_{k, i}^{t}, U_{k, i}^{t}, U_{k, i}^{t+1}$ and $\underline{x}_{k, i}^{t+1}$ compute $J_{k, i}^{t}(x)$

$$
\begin{aligned}
J_{k, i}^{t}(x) & =\left(1-\beta_{k, i}\right) \widetilde{\lambda}_{i}^{t} \widetilde{w}_{k, i}^{t} x+\left(1-\beta_{k, i}\right) \eta_{k, i} \\
& -\left(1-\beta_{k, i}\right)\left(U_{k, i}^{t}-\delta \widehat{\phi}_{i}^{t+1} U_{k, i}^{t+1}\right)+\left(1-\chi_{k, i}\right) \delta \widehat{\phi}_{i}^{t+1} \max \left\{J_{k, i}^{t+1}(x), 0\right\}
\end{aligned}
$$

- Step 4d: Solve for $\underline{x}_{k, i}^{t}: J_{k, i}^{t}\left(\underline{x}_{k, i}^{t}\right)=0$

Step 5: Compute transition rates $\left\{s_{k k^{\prime}, i}^{t, t+1}\right\}_{t=1}^{T_{S S}-1}$ for all countries $i$ according to:

$$
s_{k k^{\prime}, i}^{t, t+1}=\frac{\exp \left\{\delta \widehat{\phi}_{i}^{t+1} \theta_{k^{\prime}, i}^{t} q\left(\theta_{k^{\prime}, i}^{t}\right) \frac{\beta_{k^{\prime}, i}}{1-\beta_{k^{\prime}, i}} \int_{\underline{x}_{k^{\prime}, i}^{t+1}}^{x_{\max }} J_{k^{\prime}, i}^{t+1}(x) d G_{k^{\prime}, i}(x)+\delta \widehat{\phi}_{i}^{t+1} U_{k^{\prime}, i}^{t+1}\right\}}{\sum_{k^{\prime \prime}} \exp \left\{\delta \widehat{\phi}_{i}^{t+1} \theta_{k^{\prime \prime}, i}^{t} q\left(\theta_{k^{\prime \prime}, i}^{t}\right) \frac{\beta_{k^{\prime \prime}, i}}{1-\beta_{k^{\prime \prime}, i}} \int_{\underline{x}_{k^{\prime \prime}, i}+,}^{x_{\max }+b_{k^{\prime \prime}, i}+} J_{k^{\prime \prime}, i}^{t+1}(x) d G_{k^{\prime \prime}, i}(x)+\delta \widehat{\phi}_{i}^{t+1} U_{k^{\prime \prime}, i}^{t+1}\right\}} .
$$


Step 6: Start loop over $t$ going forward $\left(t=0\right.$ to $\left.t=T_{S S}-1\right)$

Initial conditions: we know $\widetilde{u}_{k, i}^{t=-1}=u_{k, i}^{t=0}, L_{k, i}^{t=-1}=L_{k, i}^{t=0}$, and $\theta_{k, i}^{t=0}$ from the initial steady state computation. Obtain $\widetilde{u}_{k, i}^{t}$ and $L_{k, i}^{t}$ using flow conditions and sequences $\left\{\theta_{k, i}^{t}\right\},\left\{\underline{x}_{k, i}^{t}\right\}$.

- Step 6a: Compute

$$
\begin{gathered}
J C_{k, i}^{t}=L_{k, i}^{t} u_{k, i}^{t} \theta_{k, i}^{t} q_{i}\left(\theta_{k, i}^{t}\right)\left(1-G_{k, i}\left(\underline{x}_{k, i}^{t+1}\right)\right) \\
J D_{k, i}^{t}=\left(\chi_{k, i}+\left(1-\chi_{k, i}\right) \max \left\{\frac{G_{k, i}\left(\underline{x}_{k, i}^{t+1}\right)-G_{k, i}\left(\underline{x}_{k, i}^{t}\right)}{1-G_{k, i}\left(\underline{x}_{k, i}^{t}\right)}, 0\right\}\right) L_{k, i}^{t-1}\left(1-\widetilde{u}_{k, i}^{t-1}\right) \\
\widetilde{u}_{k, i}^{t}=\frac{L_{k, i}^{t} u_{k, i}^{t}-J C_{k, i}^{t}+J D_{k, i}^{t}}{L_{k, i}^{t}}
\end{gathered}
$$

- Step 6b: Compute

$$
L_{k, i}^{t+1}=L_{k, i}^{t}+I F_{k, i}^{t+1}-O F_{k, i}^{t+1}
$$

where

$$
I F_{k, i}^{t+1}=\sum_{\ell \neq k} L_{\ell, i}^{t} \widetilde{u}_{\ell, i}^{t} s_{\ell k, i}^{t+1, t+2}
$$

and

$$
O F_{k, i}^{t+1}=L_{k, i}^{t} \widetilde{u}_{k, i}^{t}\left(1-s_{k k, i}^{t+1, t+2}\right)
$$

- Step 6c: Compute

$$
u_{k, i}^{t+1}=\frac{\sum_{\ell=1}^{K} L_{\ell, i}^{t} \widetilde{u}_{\ell, i}^{t} s_{\ell k, i}^{t+1, t+2}}{L_{k, i}^{t+1}}
$$

- Step 6d: Compute

$$
\begin{aligned}
\widetilde{L}_{k, i}^{t+1} & =L_{k, i}^{t}\left(1-\widetilde{u}_{k, i}^{t}\right) \int_{\underline{x}_{k, i}^{t+1}}^{\infty} \frac{s}{1-G_{k, i}\left(\underline{x}_{k, i}^{t+1}\right)} d G_{k, i}(s) \\
& =L_{k, i}^{t}\left(1-\widetilde{u}_{k, i}^{t}\right) \exp \left(\frac{\sigma_{k, i}^{2}}{2}\right) \frac{\Phi\left(\sigma_{k, i}-\frac{\ln \underline{x}_{k, i}^{t+1}}{\sigma_{k, i}}\right)}{\Phi\left(-\frac{\ln \underline{x}_{k, i}^{t+1}}{\sigma_{k, i}}\right)}
\end{aligned}
$$

- Step 6e: Compute expenditure with vacancies

$$
E_{k, i}^{V, t+1}=\widetilde{\kappa}_{k, i}^{t+1} \widetilde{w}_{k, i}^{t+1} \theta_{k, i}^{t+1} u_{k, i}^{t+1} L_{k, i}^{t+1}
$$


- Step 6f: Solve for $\left\{Y_{k, i}^{t+1}\right\}$ in the system

$$
\begin{gathered}
E_{k, i}^{t+1}=\mu_{k, i} E_{i}^{C, t+1}+\sum_{\ell=1}^{K}\left(\mu_{k, i} E_{\ell, i}^{V, t+1}+\left(1-\gamma_{\ell, i}\right) \nu_{\ell k, i} Y_{\ell, i}^{t+1}\right) . \\
Y_{k, o}^{t+1}=\sum_{i=1}^{N} \pi_{k, o i}^{t+1} E_{k, i}^{t+1} .
\end{gathered}
$$

- Step 6g: Compute $\left(\widetilde{w}_{k, i}^{t+1}\right)^{\prime}=\frac{\gamma_{k, i} Y_{k, i}^{t+1}}{\widetilde{L}_{k, i}^{t+1}}$

Step 7: Compute distance $\operatorname{dist}\left(\left\{\widetilde{w}_{k, i}^{t}\right\},\left\{\left(\widetilde{w}_{k, i}^{t}\right)^{\prime}\right\}\right)$

- Step 7b: Update $\widetilde{w}_{k, i}^{t}=\left(1-\lambda_{w}\right) \widetilde{w}_{k, i}^{t}+\lambda_{w}\left(\widetilde{w}_{k, i}^{t}\right)^{\prime} t=1, \ldots, T_{S S}$, for a small step size $\lambda_{w}$.

- Step 7c: At this point, we have a new series for $\left\{\widetilde{w}_{k, i}^{t}\right\}$ - go back to Step 2 until convergence of $\left\{\widetilde{w}_{k, i}^{t}\right\}$.

Step 8: Compute disposable income $\left\{I_{i}^{t}\right\}_{t=1}^{T_{S S}}$

$$
I_{i}^{t}=\sum_{\ell=1}^{K}\left(\gamma_{\ell, i} Y_{\ell, i}^{t}-E_{\ell, i}^{V, t}\right)
$$

\section{Outer Loop: iteration on $\left\{N X_{i}^{t}\right\}$}

Step 0: Impose a change in a subset of parameters that happens at $t=0$, but between $t_{c}$ and $t_{d}$. That is, the shock occurs after production, workers' decisions of where to search and after firms post vacancies at $t=0$. Impose a large value for $T_{S S}$. Assume that for $t \geq T_{S S}$ the system will have converged to a new steady state. World expenditure with final goods $\sum_{i=1}^{I} E_{i}^{C, t}$ is normalized to 1 for every $t$.

Step 1: Start with estimated state equilibrium at $t=0$. Remember that we used the normalization $\sum_{i=1}^{I} \sum_{k=1}^{K} Y_{k, i}=1$ during the estimation procedure. Change the normalization from $\sum_{i=1}^{I} \sum_{k=1}^{K} Y_{k, i}=1$ to $\sum_{i=1}^{I} E_{i}^{C}=1$. Nominal variables to be renormalized: $\left\{Y_{k, i}^{0}\right\},\left\{\widetilde{w}_{k, i}^{0}\right\},\left\{E_{i}^{C, 0}\right\},\left\{N X_{i}^{0}\right\}$.

Step 2: Obtain $B_{i}^{0}$ with respect to the normalization $\sum_{i=1}^{I} E_{i}^{C}=1$. Equation (37) gives us:

$$
B_{i}^{0}=\frac{N X_{i}^{0}}{\left(1-\frac{1}{\delta}\right)}
$$


Step 3: Make initial guess for $N X_{i}^{T_{S S}}$ (with respect to the normalization $\sum_{i=1}^{I} E_{i}^{C}=1$ ).

Step 4: Compute steady state equilibrium at $T_{S S}$, conditional on $N X_{i}^{T_{S S}}$, and the change in parameter values.

- Step 4a: Notice that the steady-state algorithm uses the normalization $\sum_{i=1}^{I} \sum_{k=1}^{K} Y_{k, i}=1$. Normalize $N X_{i}^{T_{S S}}$ with respect to normalization $\sum_{i=1}^{I} \sum_{k=1}^{K} Y_{k, i}=1$. To perform such normalization, use revenue $\left\{Y_{k, i}^{T_{S S}}\right\}$ obtained in the initial steady state if this is the first outer loop iteration, otherwise use revenue $\left\{Y_{k, i}^{T_{S S}}\right\}$ obtained in Step 6 below.

- Step 4b: After computing the final steady state, change the normalization from $\sum_{i=1}^{I} \sum_{k=1}^{K} Y_{k, i}=$ 1 to $\sum_{i=1}^{I} E_{i}^{C}=1$ using $\left\{E_{i}^{C}\right\}$ obtained in Step 3a. Nominal variables to be renormalized: $\left\{Y_{k, i}^{T_{S S}}\right\},\left\{\widetilde{w}_{k, i}^{T_{S S}}\right\},\left\{E_{i}^{C, T_{S S}}\right\},\left\{N X_{i}^{T_{S S}}\right\}$.

Step 5: Start at $t=T_{S S}-1$ and go backward until $t=1$ and sequentially compute:

$$
\begin{gathered}
R^{t+1}=\frac{1}{\delta} \frac{\sum_{i=1}^{N} \frac{E_{i}^{C, t+1}}{\widehat{\phi}_{i}^{t+1}}}{\sum_{i=1}^{N} E_{i}^{C, t}}=\frac{1}{\delta} \sum_{i=1}^{N} \frac{E_{i}^{C, t+1}}{\widehat{\phi}_{i}^{t+1}} \\
E_{i}^{C, t}=\frac{E_{i}^{C, t+1}}{\delta \widehat{\phi}_{i}^{t+1} R^{t+1}}
\end{gathered}
$$

to obtain paths for $\left\{R^{t}\right\}$ and $\left\{E_{i}^{C, t}\right\}$. Note that, because $B_{i}^{1}$ is decided at $t=0$, before the shock, $R^{1}=R^{0}=\frac{1}{\delta}$.

Step 6: Solve for the out-of-steady-state dynamics conditional on aggregate expenditures $\left\{E_{i}^{C, t}\right\}$.

Step 7: Using the path for disposable income $\left\{I_{i}^{t}\right\}_{t=1}^{T_{S S}}$ obtained in Step 6 and equation (7) compute:

$$
\begin{gathered}
\left(N X_{i}^{t}\right)^{\prime}=I_{i}^{t}-E^{C, t} \text { for } 1 \leq t<T_{S S} \\
\left(N X_{i}^{T_{S S}}\right)^{\prime}=-\frac{1-\delta}{\delta} \frac{1}{\left(\prod_{\tau=1}^{T_{S S}-1}\left(R^{\tau}\right)^{-1}\right)}\left(B_{i}^{0}+\sum_{t=1}^{T_{S S}-1}\left(\prod_{\tau=1}^{t}\left(R^{\tau}\right)^{-1}\right)\left(N X_{i}^{t}\right)^{\prime}\right)
\end{gathered}
$$

Step 8: Compute

$$
\operatorname{dist}\left(\left\{N X_{i}^{T_{S S}}\right\},\left\{\left(N X_{i}^{T_{S S}}\right)^{\prime}\right\}\right)
$$


Step 9: Update $N X_{i}^{T_{S S}}$

$$
N X_{i}^{T_{S S}}=\left(1-\lambda_{o}\right) N X_{i}^{T_{S S}}+\lambda_{o}\left(N X_{i}^{T_{S S}}\right)^{\prime}
$$

for a small step size $\lambda_{o}$ Go back to Step 4 until convergence of $\left\{N X_{i}^{T_{S S}}\right\}$. 


\section{C.5 Algorithm: Out-of-Steady-State Transition, Exogenous Deficits (No Bonds)}

Consider paths $\left\{A_{k, i}^{t}\right\}_{t=0}^{T_{S S}}$ and $\left\{d_{o, i, k}^{t}\right\}_{t=0}^{T_{S S}}$ with $A_{k, i}^{0}=1$ and $d_{o, i, k}^{0}=1$. Also, consider paths $\left\{\phi_{i}^{t}\right\}_{t=0}^{T_{S S}}$ with $\phi_{i}^{0}=1$ and $\widehat{\phi}_{i}^{t}=1$ for $T \leq t \leq T_{S S}$, for some $T<<T_{S S}$.

We condition on an exogenous path for $\left\{N X_{i}^{t}\right\}_{t=1}^{T_{S S}}$.

Step 1: Guess paths $\left\{\widetilde{\lambda}_{i}^{t}\right\}_{t=1}^{T_{S S}}$ for each country $i$.

Step 2: Guess paths $\left\{\widetilde{w}_{k, i}^{t}\right\}_{t=1}^{T_{S S}}$ for each sector $k$ and country $i$.

Step 3: Compute $\underline{x}_{k, i}^{T_{S S}}$ consistent with $\widetilde{w}_{k, i}^{T_{S S}}$ and $\widetilde{\lambda}_{i}^{T_{S S}}$. Obtain $\theta_{k, i}^{T_{S S}}, U_{k, i}^{T_{S S}}, s_{k \ell, i}^{T_{S S}, T_{S S}+1}$ and $\pi_{k, o i}^{T_{S S}}$.

- Step 3a: Compute $\widehat{\widetilde{w}}_{k, i}=\frac{\widetilde{w}_{k, i}^{T_{S S}}}{\widehat{w}_{k, i}^{0}}, \widehat{A}_{k, i}=\frac{A_{k, i}^{T_{S S}}}{A_{k, i}^{0}}$ and $\widehat{d}_{k, i}=\frac{d_{o, i, k}^{T_{S S}}}{d_{o, i, k}^{0}}$. Iteratively solve for $\widehat{P}_{k, i}^{I}$ and $\widehat{c}_{k, i}$ using the system

$$
\begin{gathered}
\widehat{c}_{k, i}=\left(\widehat{\widetilde{w}}_{k, i}\right)^{\gamma_{k, i}} \prod_{\ell=1}^{K}\left(\widehat{P}_{\ell, i}^{I}\right)\left(1-\gamma_{k, i}\right) \nu_{k \ell, i} \\
\widehat{P}_{k, i}^{I}=\left(\sum_{o=1}^{N} \pi_{k, o i}^{0} \widehat{A}_{k, o}\left(\widehat{c}_{k, o} \widehat{d}_{k, o i}\right)^{-\lambda}\right)^{-1 / \lambda}
\end{gathered}
$$

- Step 3b: Compute $\widehat{P}_{k, i}^{F}$ :

$$
\widehat{P}_{i}^{F}=\prod_{k=1}^{K}\left(\widehat{P}_{k, i}^{I}\right)^{\mu_{k i}}
$$

- Step 3c: Compute

$$
\widehat{\pi}_{k, o i}=\widehat{A}_{k, o}\left(\frac{\widehat{c}_{k, o} \widehat{d}_{k, o i}}{\widehat{P}_{k, i}^{I}}\right)^{-\lambda}
$$

and obtain $\pi_{k, o i}^{T_{S S}}=\pi_{k, o i}^{0} \widehat{\pi}_{k, o i}$

- Step 3d: Compute

$$
-\widetilde{\kappa}_{k, i}^{T_{S S}}=\widetilde{\kappa}_{k, i}^{0} \frac{\widehat{P}_{i}^{F}}{\widehat{\widetilde{w}}_{k, i}}
$$

- Step 3e: Guess $\left\{\underline{x}_{k, i}^{T_{S S}}\right\}$

- Step 3f: Compute

$$
\theta_{k, i}^{T_{S S}}=q_{i}^{-1}\left(\widetilde{\kappa}_{k, i}^{T_{S S}} \times \frac{1-\delta\left(1-\chi_{k, i}\right)}{\delta\left(1-\beta_{k, i}\right) I_{k, i}\left(\underline{x}_{k, i}^{T_{S S}}\right)}\right)
$$


- Step 3g: Compute Bellman Equations

$$
U_{k, i}^{T_{S S}}=\zeta_{i} \log \left(\sum_{k^{\prime}} \exp \left\{\frac{-C_{k k^{\prime}, i}+b_{k^{\prime}, i}+\theta_{k^{\prime}, i}^{T_{S} \widetilde{\kappa}_{k^{\prime}, i}} \widetilde{\lambda}_{i}^{T_{S S}} \widetilde{\lambda}_{S S}^{T_{S S}} \widetilde{w}_{k^{\prime}, i}^{T_{S S}} \frac{\beta_{k^{\prime}, i}}{\left(1-\beta_{k^{\prime}, i}\right)}+\delta U_{k^{\prime}, i}^{T_{S S}}}{\zeta_{i}}\right\}\right)
$$

- Step 3h: Compute

$$
\left(\underline{x}_{k, i}^{T_{S S}}\right)^{\prime}=\frac{(1-\delta) U_{k, i}^{T_{S S}}-\eta_{k, i}}{\widetilde{\lambda}_{i}^{T_{S S}} \widetilde{w}_{k, i}^{T_{S S}}}
$$

- Step 3i: Update $\underline{x}_{k, i}^{T_{S S}}=\left(1-\lambda_{x}\right) \underline{x}_{k, i}^{T_{S S}}+\lambda_{x}\left(\underline{x}_{k, i}^{T_{S S}}\right)^{\prime}$, for a small step size $\lambda_{x}$, and go back to Step 2d until convergence.

- Step 3j: Compute $s_{k k^{\prime}}^{T_{S S}, T_{S S}+1}$

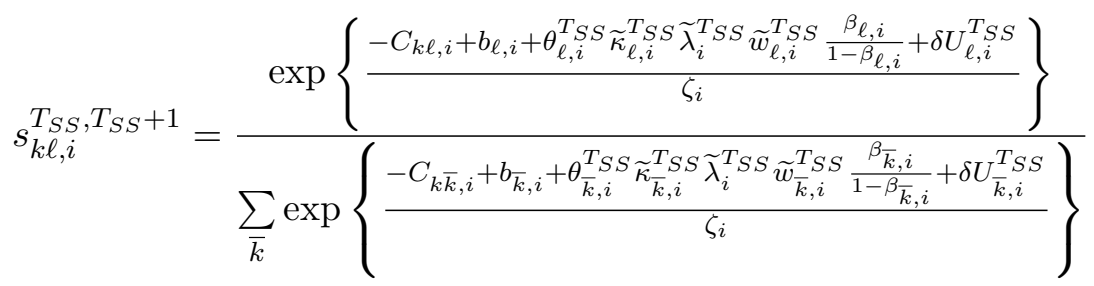

Step 4: Obtain series $\left\{\pi_{k, o i}^{t}\right\}_{t=0}^{T_{S S}},\left\{\widetilde{\kappa}_{k, i}^{t}\right\}_{t=0}^{T_{S S}}$. Define $\widehat{x}^{t} \equiv \frac{x^{t}}{x^{0}}$.

- Step 4a: For $t=1, \ldots, T_{S S}-1$ compute $\widehat{\widetilde{w}}_{k, i}^{t}=\frac{\widetilde{w}_{k, i}^{t}}{\widetilde{w}_{k, i}^{0}}$ and iteratively solve for $\widehat{P}_{k, i}^{I, t}$ and $\widehat{c}_{k, i}^{t}$ using the system

$$
\begin{aligned}
\widehat{c}_{k, i}^{t} & =\left(\widehat{\widetilde{w}}_{k, i}^{t}\right)^{\gamma_{k, i}} \prod_{\ell=1}^{K}\left(\widehat{P}_{\ell, i}^{I, t}\right)^{\left(1-\gamma_{k, i}\right) \nu_{k \ell, i}} \\
\widehat{P}_{k, i}^{I, t} & =\left(\sum_{o=1}^{N} \pi_{k, o i}^{0} \widehat{A}_{k, o}^{t}\left(\widehat{c}_{k, o}^{t} \widehat{d}_{k, o i}^{t}\right)^{-\lambda}\right)^{-1 / \lambda}
\end{aligned}
$$

- Step 4b: Compute $\widehat{P}_{k, i}^{F, t}$ for $t=1, \ldots, T_{S S}-1$ :

$$
\widehat{P}_{i}^{F, t}=\prod_{k=1}^{K}\left(\widehat{P}_{k, i}^{I, t}\right)^{\mu_{k i}}
$$

- Step 4c: Compute $\widehat{\pi}_{k, o i}^{t}$ for $t=1, \ldots, T_{S S}-1$ :

$$
\widehat{\pi}_{k, o i}^{t}=\widehat{A}_{k, o}^{t}\left(\frac{\widehat{c}_{k, o}^{t} \widehat{d}_{k, o i}^{t}}{\widehat{P}_{k, i}^{I, t}}\right)^{-\lambda}
$$

- Step 4d: Compute or $t=1, \ldots, T_{S S}-1$ : 


$$
\begin{aligned}
& -\pi_{k, o i}^{t}=\pi_{k, o i}^{0} \widehat{\pi}_{k, o i}^{t} \\
& -\widetilde{\kappa}_{k, i}^{t} \equiv \frac{\kappa_{k, i} P_{i}^{F, t}}{\widetilde{w}_{k, i}^{t}}=\frac{\kappa_{k, i} P_{i}^{F, 0}}{\widetilde{w}_{k, i}^{0}} \frac{P_{i}^{F, t}}{P_{i}^{F, 0}} \frac{\widetilde{w}_{k, i}^{0}}{\widetilde{w}_{k, i}^{t}}=\widetilde{\kappa}_{k, i}^{0} \frac{\widehat{P}_{i}^{F, t}}{\widehat{\widetilde{w}}_{k, i}^{t}}
\end{aligned}
$$

Step 5: Given knowledge of $\widetilde{w}_{k, i}^{T_{S S}}, \widetilde{\lambda}_{i}^{T_{S S}}$ and $\underline{x}_{k, i}^{T_{S S}}$ (and therefore $J_{k, i}^{T_{S S}}(s)$ ), start at $t=T_{S S}-1$ and sequentially compute (backwards) for each $t=T_{S S}-1, \ldots, 1$

- Step 5a: Given $\widetilde{w}_{k, i}^{t}, \widetilde{\lambda}_{i}^{t}, \underline{x}_{k, i}^{t+1}, \widetilde{\kappa}_{k, i}^{t}$ and $J_{k, i}^{t+1}(s)$ compute $\theta_{k, i}^{t}$.

If $\frac{\widetilde{\lambda}_{i}^{t} \widetilde{\kappa}_{k, i}^{t} \widetilde{w}_{k, i}^{t}}{\delta \widehat{\phi}_{i}^{t+1} \int_{\underline{x}_{k, i}^{t+1}}^{x+1} J_{k, i}^{t+1}(s) d G_{k, i}(s)} \leq 1$ then

$$
\theta_{k, i}^{t}=q_{i}^{-1}\left(\frac{\widetilde{\lambda}_{i}^{t} \widetilde{\kappa}_{k, i}^{t} \widetilde{w}_{k, i}^{t}}{\delta \widehat{\phi}_{i}^{t+1} \int_{\underline{x}_{k, i}^{t+1}}^{x_{\max }} J_{k, i}^{t+1}(s) d G_{k, i}(s)}\right)
$$

If $\frac{\widetilde{\lambda}_{i}^{t} \widetilde{\kappa}_{k, i}^{t} \widetilde{w}_{k, i}^{t}}{\delta \widehat{\phi}_{i}^{t+1} \int_{\underline{x}_{k, i}^{t+1}}^{x_{\max }} J_{k, i}^{t+1}(s) d G_{k, i}(s)}>1$, it is not possible to satisfy $V_{k, i}^{t}=0$, so that $V_{k, i}^{t}<0$ and

$$
\theta_{k, i}^{t}=0
$$

- Step 5b: Given $\underline{x}_{k, i}^{t+1}, W_{k, i}^{t+1}(x)=\frac{\beta_{k, i}}{1-\beta_{k, i}} J_{k, i}^{t+1}(x)+U_{k, i}^{t+1}\left(\right.$ for $\left.x \geq \underline{x}_{k, i}^{t+1}\right), \theta_{k, i}^{t}, U_{k, i}^{t+1}$ compute $U_{k, i}^{t}$. Notice that $\int_{\underline{x}_{k, i}^{t+1}}^{x_{\max }} W_{k, i}^{t+1}(s) d G_{k, i}(s)=\frac{\beta_{k, i}}{1-\beta_{k, i}} \int_{\underline{x}_{k, i}^{t+1}}^{x_{\max }} J_{k, i}^{t+1}(s) d G_{k, i}(s)+\left(1-G_{k, i}\left(\underline{x}_{k, i}^{t+1}\right)\right) U_{k, i}^{t+1}$ so that:

$$
U_{k, i}^{t}=\zeta_{i} \log \left(\sum_{k^{\prime}} \exp \left\{\frac{+\delta \widehat{\phi}_{i}^{t+1} \theta_{k^{\prime}, i}^{t} q_{i}\left(\theta_{k^{\prime}, i}^{t}\right) \frac{\beta_{k, i}}{1-\beta_{k, i}} \int_{\underline{x}_{k, i}^{t+1}}^{x_{\text {max }}} J_{k, i}^{t+1}(s) d G_{k, i}(s)+\delta \widehat{\phi}_{i}^{t+1} U_{k^{\prime}, i}^{t+1}}{\zeta_{i}}\right\}\right)
$$

- Step 5c: Given $\widetilde{\lambda}_{i}^{t}, J_{k, i}^{t+1}(x), \widetilde{w}_{k, i}^{t}, \theta_{k, i}^{t}, \delta, U_{k, i}^{t}, U_{k, i}^{t+1}$ and $\underline{x}_{k, i}^{t+1}$ compute $J_{k, i}^{t}(x)$

$$
\begin{aligned}
J_{k, i}^{t}(x) & =\left(1-\beta_{k, i}\right) \widetilde{\lambda}_{i}^{t} \widetilde{w}_{k, i}^{t} x+\left(1-\beta_{k, i}\right) \eta_{k, i} \\
& -\left(1-\beta_{k, i}\right)\left(U_{k, i}^{t}-\delta \widehat{\phi}_{i}^{t+1} U_{k, i}^{t+1}\right)+\left(1-\chi_{k, i}\right) \delta \widehat{\phi}_{i}^{t+1} \max \left\{J_{k, i}^{t+1}(x), 0\right\}
\end{aligned}
$$

- Step 5d: Solve for $\underline{x}_{k, i}^{t}: J_{k, i}^{t}\left(\underline{x}_{k, i}^{t}\right)=0$

Step 6: Compute transition rates $\left\{s_{k k^{\prime}, i}^{t, t+1}\right\}_{t=1}^{T_{S S}-1}$ for all countries $i$ according to:

$$
s_{k k^{\prime}, i}^{t, t+1}=\frac{\exp \left\{\delta \widehat{\phi}_{i}^{t+1} \theta_{k^{\prime}, i}^{t} q\left(\theta_{k^{\prime}, i}^{t}\right) \frac{\beta_{k^{\prime}, i}}{1-\beta_{k^{\prime}, i}} \int_{\underline{x}_{k^{\prime}, i}^{t+1}}^{x_{\max }} J_{k^{\prime}, i}^{t+1}(x) d G_{k^{\prime}, i}(x)+\delta \widehat{\phi}_{i}^{t+1} U_{k^{\prime}, i}^{t+1}\right\}}{\sum_{k^{\prime \prime}} \exp \left\{\delta \widehat{\phi}_{i}^{t+1} \theta_{k^{\prime \prime}, i}^{t} q\left(\theta_{k^{\prime \prime}, i}^{t}\right) \frac{\beta_{k^{\prime \prime}, i}}{1-\beta_{k^{\prime \prime}, i}} \int_{\underline{x}_{k^{\prime \prime}, i}}^{x_{\max }+1} J_{k^{\prime \prime}, i}^{t+1}(x) d G_{k^{\prime \prime}, i}(x)+\delta \widehat{\phi}_{i}^{t+1} U_{k^{\prime \prime}, i}^{t+1}\right\}} .
$$


Step 7: Start loop over $t$ going forward $\left(t=0\right.$ to $\left.t=T_{S S}-1\right)$

Initial conditions: we know $\widetilde{u}_{k, i}^{t=-1}=u_{k, i}^{t=0}, L_{k, i}^{t=-1}=L_{k, i}^{t=0}$, and $\theta_{k, i}^{t=0}$ from the initial steady state computation. Obtain $\widetilde{u}_{k, i}^{t}$ and $L_{k, i}^{t}$ using flow conditions and sequences $\left\{\theta_{k, i}^{t}\right\},\left\{\underline{x}_{k, i}^{t}\right\}$.

- Step 7a: Compute

$$
\begin{gathered}
J C_{k, i}^{t}=L_{k, i}^{t} u_{k, i}^{t} \theta_{k, i}^{t} q_{i}\left(\theta_{k, i}^{t}\right)\left(1-G_{k, i}\left(\underline{x}_{k, i}^{t+1}\right)\right) \\
J D_{k, i}^{t}=\left(\chi_{k, i}+\left(1-\chi_{k, i}\right) \max \left\{\frac{G_{k, i}\left(\underline{x}_{k, i}^{t+1}\right)-G_{k, i}\left(\underline{x}_{k, i}^{t}\right)}{1-G_{k, i}\left(\underline{x}_{k, i}^{t}\right)}, 0\right\}\right) L_{k, i}^{t-1}\left(1-\widetilde{u}_{k, i}^{t-1}\right) \\
\widetilde{u}_{k, i}^{t}=\frac{L_{k, i}^{t} u_{k, i}^{t}-J C_{k, i}^{t}+J D_{k, i}^{t}}{L_{k, i}^{t}}
\end{gathered}
$$

- Step 7b: Compute

$$
L_{k, i}^{t+1}=L_{k, i}^{t}+I F_{k, i}^{t+1}-O F_{k, i}^{t+1}
$$

where

$$
I F_{k, i}^{t+1}=\sum_{\ell \neq k} L_{\ell, i}^{t} \widetilde{u}_{\ell, i}^{t} s_{\ell k, i}^{t+1, t+2}
$$

and

$$
O F_{k, i}^{t+1}=L_{k, i}^{t} \widetilde{u}_{k, i}^{t}\left(1-s_{k k, i}^{t+1, t+2}\right)
$$

- Step 7c: Compute

$$
u_{k, i}^{t+1}=\frac{\sum_{\ell=1}^{K} L_{\ell, i}^{t} \widetilde{u}_{\ell, i}^{t} s_{\ell k, i}^{t+1, t+2}}{L_{k, i}^{t+1}}
$$

- Step 7d: Compute

$$
\begin{aligned}
\widetilde{L}_{k, i}^{t+1} & =L_{k, i}^{t}\left(1-\widetilde{u}_{k, i}^{t}\right) \int_{\underline{x}_{k, i}^{t+1}}^{\infty} \frac{s}{1-G_{k, i}\left(\underline{x}_{k, i}^{t+1}\right)} d G_{k, i}(s) \\
& =L_{k, i}^{t}\left(1-\widetilde{u}_{k, i}^{t}\right) \exp \left(\frac{\sigma_{k, i}^{2}}{2}\right) \frac{\Phi\left(\sigma_{k, i}-\frac{\ln \underline{x}_{k, i}^{t+1}}{\sigma_{k, i}}\right)}{\Phi\left(-\frac{\ln \underline{x}_{k, i}^{t+1}}{\sigma_{k, i}}\right)}
\end{aligned}
$$

and $Y_{k, i}^{t+1}=\widetilde{w}_{k, i}^{t+1} \widetilde{L}_{k, i}^{t+1}$ 
- Step 7e: Compute expenditure with vacancies

$$
E_{k, i}^{V, t+1}=\widetilde{\kappa}_{k, i}^{t+1} \widetilde{w}_{k, i}^{t+1} \theta_{k, i}^{t+1} u_{k, i}^{t+1} L_{k, i}^{t+1}
$$

- Step 7f: Compute $E_{i}^{C, t+1}=\frac{\bar{L}_{i}}{\tilde{\lambda}_{i}^{t+1}}$

- Step 7g: Solve for $\left\{Y_{k, i}^{t+1}\right\}$ in the system

$$
\begin{gathered}
E_{k, i}^{t+1}=\mu_{k, i} E_{i}^{C, t+1}+\sum_{\ell=1}^{K}\left(\mu_{k, i} E_{\ell, i}^{V, t+1}+\left(1-\gamma_{\ell, i}\right) \nu_{\ell k, i} Y_{\ell, i}^{t+1}\right) . \\
Y_{k, o}^{t+1}=\sum_{i=1}^{N} \pi_{k, o i}^{t+1} E_{k, i}^{t+1} .
\end{gathered}
$$

- Step 7h: Normalize $\left\{Y_{k, i}^{t+1}\right\}$ to make sure it sums to 1 across sectors and countries.

- Step 7i: Compute $\left(\widetilde{w}_{k, i}^{t+1}\right)^{\prime}=\frac{\gamma_{k, i} Y_{k, i}^{t+1}}{\widetilde{L}_{k, i}^{t+1}}$

Step 8: Compute $\operatorname{dist}\left(\left\{\widetilde{w}_{k, i}^{t}\right\}_{t=1}^{T_{S S}},\left\{\left(\widetilde{w}_{k, i}^{t}\right)^{\prime}\right\}_{t=1}^{T_{S S}}\right)$

Step 9: Update $\widetilde{w}_{k, i}^{t}=\left(1-\alpha_{w}\right) \widetilde{w}_{k, i}^{t}+\alpha_{w}\left(\widetilde{w}_{k, i}^{t}\right)^{\prime}$ for $t=1, \ldots, T_{S S}$, for a small step size $\alpha_{w}$, and go back to Step 3 until convergence of $\left\{\widetilde{w}_{k, i}^{t}\right\}$

Step 10: Compute disposable income $\left\{I_{i}^{t}\right\}_{t=1}^{T_{S S}}$

$$
I_{i}^{t}=\sum_{\ell=1}^{K}\left(\gamma_{\ell, i} Y_{\ell, i}^{t}-E_{\ell, i}^{V, t}\right)
$$

Step 11: Update $\left\{E_{i}^{C, t}\right\}_{t=1}^{T_{S S}}$ using

$$
E_{i}^{C, t}=I_{i}^{t}-N X_{i}^{t}
$$

Step 12a: Compute $\left(\widetilde{\lambda}_{i}^{t}\right)^{\prime}=\frac{\bar{L}_{i}}{E_{i}^{C, t}}$ for all $t=1, \ldots, T_{S S}$

- Step 12b: Compute dist $\left(\left\{\widetilde{\lambda}_{i}^{t}\right\}_{t=1}^{T_{S S}},\left\{\left(\widetilde{\lambda}_{i}^{t}\right)^{\prime}\right\}_{t=1}^{T_{S S}}\right)$

- Step 12c: Update $\widetilde{\lambda}_{i}^{t}=\left(1-\alpha_{\lambda}\right) \widetilde{\lambda}_{i}^{t}+\alpha_{\lambda}\left(\widetilde{\lambda}_{i}^{t}\right)^{\prime}$ for $t=1, \ldots, T_{S S}$, for a small step size $\alpha_{\lambda}$, and go back to Step 2 until convergence of $\left\{\tilde{\lambda}_{i}^{t}\right\}$. 


\section{C.6 Algorithm: Recovering Shocks}

Inner Loop: conditional on paths for expenditures $\left\{E_{i}^{C, t}\right\}_{t=1}^{T_{S S}}$ and shocks $\left\{\widehat{\phi}_{i}^{t}\right\}_{t=2}^{T_{S S}},\left\{\widehat{d}_{k, i}^{t}\right\}_{t=1}^{T_{S S}}$ and $\widehat{A}_{k, i} \equiv \frac{A_{k, i}^{T} T_{k, i}}{A_{k, i}^{0}}-$ determined in the Outer Loop below.

As before, we denote changes relative to $t=0$ by $\widehat{x}^{t}=\frac{x^{t}}{x^{0}}$. This loop conditions on data on $\left\{\widehat{\pi}_{k, o i}^{t}\right\}_{t=1}^{T}$ and $\left\{\widehat{P}_{k, i}^{I, t}\right\}_{t=1}^{T}$, where $T$ is the last period we have data on these variables. We assume $t=0$ is the estimated steady state. Define $\widehat{d}_{k, i} \equiv \frac{d_{o, i, k}^{T}}{d_{o, i, k}^{0}}$.

Step 1: Given paths $\left\{E_{i}^{C, t}\right\}$, compute paths $\left\{\widetilde{\lambda}_{i}^{t}\right\}: \widetilde{\lambda}_{i}^{t}=\frac{\bar{L}_{i}}{E_{i}^{C, t}}$.

Step 2: Guess paths $\left\{\widetilde{w}_{k, i}^{t}\right\}_{t=1}^{T_{S S}}$ for each sector $k$ and country $i$.

Step 3: Compute $\underline{x}_{k, i}^{T_{S S}}$ consistent with $\widetilde{w}_{k, i}^{T_{S S}}$ and $\widetilde{\lambda}_{i}^{T_{S S}}$. Obtain $\theta_{k, i}^{T_{S S}}, U_{k, i}^{T_{S S}}, s_{k \ell, i}^{T_{S S}, T_{S S}+1}$ and $\pi_{k, o i}^{T_{S S}}$.

- Step 3a: Compute $\widehat{\widetilde{w}}_{k, i}=\frac{\widetilde{w}_{k, i}^{T} \widehat{w}_{k, i}^{0}}{\widetilde{w}_{k, i}^{0}}$ Iteratively solve for $\widehat{P}_{k, i}^{I}$ and $\widehat{c}_{k, i}$ using the system

$$
\begin{gathered}
\widehat{c}_{k, i}=\left(\widehat{\widetilde{w}}_{k, i}\right)^{\gamma_{k, i}} \prod_{\ell=1}^{K}\left(\widehat{P}_{\ell, i}^{I}\right)^{\left(1-\gamma_{k, i}\right) \nu_{k \ell, i}} \\
\widehat{P}_{k, i}^{I}=\left(\sum_{o=1}^{N} \pi_{k, o i}^{0} \widehat{A}_{k, o}\left(\widehat{c}_{k, o} \widehat{d}_{k, o i}\right)^{-\lambda}\right)^{-1 / \lambda}
\end{gathered}
$$

- Step 3b: Compute $\widehat{P}_{k, i}^{F}$ :

$$
\widehat{P}_{i}^{F}=\prod_{k=1}^{K}\left(\widehat{P}_{k, i}^{I}\right)^{\mu_{k i}}
$$

- Step 3c: Compute

$$
\widehat{\pi}_{k, o i}=\widehat{A}_{k, o}\left(\frac{\widehat{c}_{k, o} \widehat{d}_{k, o i}}{\widehat{P}_{k, i}^{I}}\right)^{-\lambda}
$$

And obtain $\pi_{k, o i}^{T_{S S}}=\pi_{k, o i}^{0} \widehat{\pi}_{k, o i}$

- Step 3d: Compute

$$
-\widetilde{\kappa}_{k, i}^{T_{S S}}=\widetilde{\kappa}_{k, i}^{0} \frac{\widehat{P}_{i}^{F}}{\widehat{\widetilde{w}}_{k, i}}
$$

- Step 3e: Guess $\left\{\underline{x}_{k, i}^{T_{S S}}\right\}$ 
- Step 3f: Compute

$$
\theta_{k, i}^{T_{S S}}=q_{i}^{-1}\left(\widetilde{\kappa}_{k, i}^{T_{S S}} \times \frac{1-\delta\left(1-\chi_{k, i}\right)}{\delta\left(1-\beta_{k, i}\right) I_{k, i}\left(\underline{x}_{k, i}^{T_{S S}}\right)}\right)
$$

- Step 3g: Compute Bellman Equations

$$
U_{k, i}^{T_{S S}}=\zeta_{i} \log \left(\sum_{k^{\prime}} \exp \left\{\frac{-C_{k k^{\prime}, i}+b_{k^{\prime}, i}+\theta_{k^{\prime}, i}^{T_{S S}} \widetilde{\kappa}_{k^{\prime}, i}^{T_{S S}} \widetilde{\lambda}_{i}^{T_{S S}} \widetilde{w}_{k^{\prime}, i}^{T_{S S}} \frac{\beta_{k^{\prime}, i}}{\left(1-\beta_{k^{\prime}, i}\right)}+\delta U_{k^{\prime}, i}^{T_{S S}}}{\zeta_{i}}\right\}\right)
$$

- Step 3h: Compute

$$
\left(\underline{x}_{k, i}^{T_{S S}}\right)^{\prime}=\frac{(1-\delta) U_{k, i}^{T_{S S}}-\eta_{k, i}}{\widetilde{\lambda}_{i}^{T_{S S}} \widetilde{w}_{k, i}^{T_{S S}}}
$$

- Step 3i: Update $\underline{x}_{k, i}^{T_{S S}}=\left(1-\lambda_{x}\right) \underline{x}_{k, i}^{T_{S S}}+\lambda_{x}\left(\underline{x}_{k, i}^{T_{S S}}\right)^{\prime}$, for a small step size $\lambda_{x}$ and go back to Step 2d until convergence.

- Step 3j: Compute $s_{k k^{\prime}}^{T_{S S}, T_{S S}+1}$

$$
s_{k \ell, i}^{T_{S S}, T_{S S}+1}=\frac{\exp \left\{\frac{-C_{k \ell, i}+b_{\ell, i}+\theta_{\ell, i}^{T_{S S}} \widetilde{\kappa}_{\ell, i}^{T_{S S}} \widetilde{\lambda}_{i}^{T} \widetilde{w}_{S S} \widetilde{w}_{\ell, i}^{T_{S S}} \frac{\beta_{\ell, i}}{1-\beta_{\ell, i}}+\delta U_{\ell, i}^{T_{S S}}}{\zeta_{i}}\right\}}{\sum_{\bar{k}} \exp \left\{\frac{-C_{k \bar{k}, i}+b_{\bar{k}, i}+\theta_{\bar{k}, i}^{T_{S S}} \widetilde{\kappa}_{\bar{k}, i}^{T_{S S}} \widetilde{\lambda}_{i}^{T} S S \widetilde{w}_{\bar{k}, i}^{T_{S S}} \frac{\beta_{\bar{k}, i}}{1-\beta_{\bar{k}, i}}+\delta U_{\bar{k}, i}^{T_{S S}}}{\zeta_{i}}\right\}}
$$

Step 4: Obtain series $\left\{\pi_{k, o i}^{t}\right\}_{t=T+1}^{T_{S S}}$ and $\left\{\widetilde{\kappa}_{k, i}^{t}\right\}_{t=1}^{T_{S S}}$.

- Step 4a: For $t=T+1, \ldots, T_{S S}$ do:

Compute $\widehat{\widetilde{w}}_{k, i}^{t}=\frac{\widetilde{w}_{k, i}^{t}}{\widetilde{w}_{k, i}^{0}}$ and iteratively solve for $\widehat{P}_{k, i}^{I, t}$ and $\widehat{c}_{k, i}^{t}$ using the system

$$
\begin{gathered}
\widehat{c}_{k, i}^{t}=\left(\widehat{\widetilde{w}}_{k, i}^{t}\right)^{\gamma_{k, i}} \prod_{\ell=1}^{K}\left(\widehat{P}_{\ell, i}^{I, t}\right)^{\left(1-\gamma_{k, i}\right) \nu_{k \ell, i}}, \\
\widehat{P}_{k, i}^{I, t}=\left(\sum_{o=1}^{N} \pi_{k, o i}^{0} \widehat{A}_{k, o}\left(\widehat{c}_{k, o}^{t} \widehat{d}_{k, o i}\right)^{-\lambda}\right)^{-1 / \lambda} .
\end{gathered}
$$

- Step 4b: Compute $\widehat{P}_{k, i}^{F, t}$ for $t=1, \ldots, T_{S S}-1$ (remember $\widehat{P}_{k, i}^{I, t}$ is data for $\left.t=1, \ldots, T\right)$ :

$$
\widehat{P}_{i}^{F, t}=\prod_{k=1}^{K}\left(\widehat{P}_{k, i}^{I, t}\right)^{\mu_{k i}}
$$


- Step 4c: Compute $\widehat{\pi}_{k, o i}^{t}$ and $\pi_{k, o i}^{t}$ for $t=T+1, \ldots, T_{S S}-1$.

For $t=1, \ldots, T_{S S}-1$ do:

First Case: If $t \leq T$ then $\widehat{\pi}_{k, o i}^{t}$ is data, so do:

$$
\pi_{k, o i}^{t}=\pi_{k, o i}^{0} \widehat{\pi}_{k, o i}^{t}
$$

End of First Case

Second Case if $t \geq T+1$ do:

$$
\begin{gathered}
\widehat{\pi}_{k, o i}^{t}=\left(\widehat{A}_{k, o}^{t}\right)^{\prime}\left(\frac{\widehat{c}_{k, o}^{t} \widehat{d}_{k, o i}^{t}}{\widehat{P}_{k, i}^{I, t}}\right)^{-\lambda} \\
\pi_{k, o i}^{t}=\pi_{k, o i}^{0} \widehat{\pi}_{k, o i}^{t}
\end{gathered}
$$

End of Second Case

- Step 4d: Compute for $t=1, \ldots, T_{S S}-1$

$$
-\widetilde{\kappa}_{k, i}^{t} \equiv \frac{\kappa_{k, i} P_{i}^{F, t}}{\widetilde{w}_{k, i}^{t}}=\frac{\kappa_{k, i} P_{i}^{F, 0}}{\widetilde{w}_{k, i}^{0}} \frac{P_{i}^{F, t}}{P_{i}^{F, 0}} \frac{\widetilde{w}_{k, i}^{0}}{\widetilde{w}_{k, i}^{t}}=\widetilde{\kappa}_{k, i}^{0} \frac{\widehat{P}_{i}^{F, t}}{\widehat{\widetilde{w}}_{k, i}^{t}}
$$

Step 5: Given knowledge of $\widetilde{w}_{k, i}^{T_{S S}}, \widetilde{\lambda}_{i}^{T_{S S}}$ and $\underline{x}_{k, i}^{T_{S S}}$ (and therefore $J_{k, i}^{T_{S S}}(s)$ ), start at $t=T_{S S}-1$ and sequentially compute (backwards) for each $t=T_{S S}-1, \ldots, 1$

- Step 5a: Given $\widetilde{w}_{k, i}^{t}, \underline{x}_{k, i}^{t+1}, \widetilde{\kappa}_{k, i}^{t}, \widetilde{\lambda}_{i}^{t}$ and $J_{k, i}^{t+1}(s)$ compute $\theta_{k, i}^{t}$.

If $\frac{\widetilde{\lambda}_{i}^{t} \widetilde{\kappa}_{k, i}^{t} \widetilde{w}_{k, i}^{t}}{\delta \bar{\phi}_{i}^{t+1} \int_{\underline{x}_{k, i}^{t+1}}^{x \rightarrow+1} J_{k, i}^{t+1}(s) d G_{k, i}(s)} \leq 1$ then

$$
\theta_{k, i}^{t}=q_{i}^{-1}\left(\frac{\widetilde{\lambda}_{i}^{t} \widetilde{\kappa}_{k, i}^{t} \widetilde{w}_{k, i}^{t}}{\delta \widehat{\phi}_{i}^{t+1} \int_{\underline{x}_{k, i}^{t+1}}^{x_{\max }} J_{k, i}^{t+1}(s) d G_{k, i}(s)}\right)
$$

If $\frac{\widetilde{\lambda}_{i}^{t} \widetilde{\kappa}_{k, i}^{t} \widetilde{w}_{k, i}^{t}}{\delta \widehat{\phi}_{i}^{t+1} \int_{\underline{x}_{k, i}^{t+1}}^{x_{\max }} J_{k, i}^{t+1}(s) d G_{k, i}(s)}>1$, it is not possible to satisfy $V_{k, i}^{t}=0$, so that $V_{k, i}^{t}<0$ and

$$
\theta_{k, i}^{t}=0
$$

- Step 5b: Given $\underline{x}_{k, i}^{t+1}, W_{k, i}^{t+1}(x)=\frac{\beta_{k, i}}{1-\beta_{k, i}} J_{k, i}^{t+1}(x)+U_{k, i}^{t+1}\left(\right.$ for $\left.x \geq \underline{x}_{k, i}^{t+1}\right), \theta_{k, i}^{t}, U_{k, i}^{t+1}$ compute $U_{k, i}^{t}$. Notice that $\int_{\underline{x}_{k, i}^{t+1}}^{x_{\max }} W_{k, i}^{t+1}(s) d G_{k, i}(s)=\frac{\beta_{k, i}}{1-\beta_{k, i}} \int_{\underline{x}_{k, i}^{t+1}}^{x_{\max }} J_{k, i}^{t+1}(s) d G_{k, i}(s)+\left(1-G_{k, i}\left(\underline{x}_{k, i}^{t+1}\right)\right) U_{k, i}^{t+1}$ so that:

$$
U_{k, i}^{t}=\zeta_{i} \log \left(\sum_{k^{\prime}} \exp \left\{\frac{+\delta \widehat{\phi}_{i}^{t+1} \theta_{k^{\prime}, i}^{t} q_{i}\left(\theta_{k^{\prime}, i}^{t}\right) \frac{\beta_{k, i}}{1-\beta_{k, i}} \int_{\underline{x}_{k, i}^{t+1}}^{x_{\text {max }}} J_{k, i}^{t+1}(s) d G_{k, i}(s)+\delta \widehat{\phi}_{i}^{t+1} U_{k^{\prime}, i}^{t+1}}{\zeta_{i}}\right\}\right)
$$


- Step 5c: Given $J_{k, i}^{t+1}(x), \widetilde{w}_{k, i}^{t}, U_{k, i}^{t}, U_{k, i}^{t+1}$ and $\underline{x}_{k, i}^{t+1}$ compute $J_{k, i}^{t}(x)$

$$
\begin{aligned}
J_{k, i}^{t}(x) & =\left(1-\beta_{k, i}\right) \widetilde{\lambda}_{i}^{t} \widetilde{w}_{k, i}^{t} x+\left(1-\beta_{k, i}\right) \eta_{k, i} \\
& -\left(1-\beta_{k, i}\right)\left(U_{k, i}^{t}-\delta \widehat{\phi}_{i}^{t+1} U_{k, i}^{t+1}\right)+\left(1-\chi_{k, i}\right) \delta \widehat{\phi}_{i}^{t+1} \max \left\{J_{k, i}^{t+1}(x), 0\right\}
\end{aligned}
$$

- Step 5d: Solve for $\underline{x}_{k, i}^{t}: J_{k, i}^{t}\left(\underline{x}_{k, i}^{t}\right)=0$

Step 6: Compute transition rates $\left\{s_{k k^{\prime}, i}^{t, t+1}\right\}_{t=1}^{T_{S S}-1}$ for all countries $i$ according to:

$$
s_{k k^{\prime}, i}^{t, t+1}=\frac{\exp \left\{\delta \widehat{\phi}_{i}^{t+1} \theta_{k^{\prime}, i}^{t} q\left(\theta_{k^{\prime}, i}^{t}\right) \frac{\beta_{k^{\prime}, i}}{1-\beta_{k^{\prime}, i}} \int_{\underline{x}_{k^{\prime}, i}^{t+1}}^{x_{\max }} J_{k^{\prime}, i}^{t+1}(x) d G_{k^{\prime}, i}(x)+\delta \widehat{\phi}_{i}^{t+1} U_{k^{\prime}, i}^{t+1}\right\}}{\sum_{k^{\prime \prime}} \exp \left\{\delta \widehat{\phi}_{i}^{t+1} \theta_{k^{\prime \prime}, i}^{t} q\left(\theta_{k^{\prime \prime}, i}^{t}\right) \frac{\beta_{k^{\prime \prime}, i}}{-C_{k k^{\prime \prime}, i}+b_{k^{\prime \prime}, i}} \int_{\underline{k}_{k^{\prime \prime}, i}+1}^{x_{\max }+1} J_{k^{\prime \prime}, i}^{t+1}(x) d G_{k^{\prime \prime}, i}(x)+\delta \widehat{\phi}_{i}^{t+1} U_{k^{\prime \prime}, i}^{t+1}\right\}} .
$$

Step 7: Start loop over $t$ going forward $\left(t=0\right.$ to $\left.t=T_{S S}-1\right)$

Initial conditions: we know $\widetilde{u}_{k, i}^{t=-1}=u_{k, i}^{t=0}, L_{k, i}^{t=-1}=L_{k, i}^{t=0}$, and $\theta_{k, i}^{t=0}$ from the initial steady state computation. Obtain $\widetilde{u}_{k, i}^{t}$ and $L_{k, i}^{t}$ using flow conditions and sequences $\left\{\theta_{k, i}^{t}\right\},\left\{\underline{x}_{k, i}^{t}\right\}$.

- Step 7a: Compute

$$
\begin{gathered}
J C_{k, i}^{t}=L_{k, i}^{t} u_{k, i}^{t} \theta_{k, i}^{t} q_{i}\left(\theta_{k, i}^{t}\right)\left(1-G_{k, i}\left(\underline{x}_{k, i}^{t+1}\right)\right) \\
J D_{k, i}^{t}=\left(\chi_{k, i}+\left(1-\chi_{k, i}\right) \max \left\{\frac{G_{k, i}\left(\underline{x}_{k, i}^{t+1}\right)-G_{k, i}\left(\underline{x}_{k, i}^{t}\right)}{1-G_{k, i}\left(\underline{x}_{k, i}^{t}\right)}, 0\right\}\right) L_{k, i}^{t-1}\left(1-\widetilde{u}_{k, i}^{t-1}\right) \\
\widetilde{u}_{k, i}^{t}=\frac{L_{k, i}^{t} u_{k, i}^{t}-J C_{k, i}^{t}+J D_{k, i}^{t}}{L_{k, i}^{t}}
\end{gathered}
$$

- Step 7b: Compute

$$
L_{k, i}^{t+1}=L_{k, i}^{t}+I F_{k, i}^{t+1}-O F_{k, i}^{t+1},
$$

where

$$
I F_{k, i}^{t+1}=\sum_{\ell \neq k} L_{\ell, i}^{t} \widetilde{u}_{\ell, i}^{t} s_{\ell k, i}^{t+1, t+2}
$$

and

$$
O F_{k, i}^{t+1}=L_{k, i}^{t} \widetilde{u}_{k, i}^{t}\left(1-s_{k k, i}^{t+1, t+2}\right)
$$


- Step 7c: Compute

$$
u_{k, i}^{t+1}=\frac{\sum_{\ell=1}^{K} L_{\ell, i}^{t} \widetilde{u}_{\ell, i}^{t} s_{\ell k, i}^{t+1, t+2}}{L_{k, i}^{t+1}}
$$

- Step 7d: Compute

$$
\begin{aligned}
\widetilde{L}_{k, i}^{t+1} & =L_{k, i}^{t}\left(1-\widetilde{u}_{k, i}^{t}\right) \int_{\underline{x}_{k, i}^{t+1}}^{\infty} \frac{s}{1-G_{k, i}\left(\underline{x}_{k, i}^{t+1}\right)} d G_{k, i}(s) \\
& =L_{k, i}^{t}\left(1-\widetilde{u}_{k, i}^{t}\right) \exp \left(\frac{\sigma_{k, i}^{2}}{2}\right) \frac{\Phi\left(\sigma_{k, i}-\frac{\ln \underline{x}_{k, i}^{t+1}}{\sigma_{k, i}}\right)}{\Phi\left(-\frac{\ln \underline{x}_{k, i}^{t+1}}{\sigma_{k, i}}\right)}
\end{aligned}
$$

- Step 7e: Compute expenditure with vacancies

$$
E_{k, i}^{V, t+1}=\widetilde{\kappa}_{k, i}^{t+1} \widetilde{w}_{k, i}^{t+1} \theta_{k, i}^{t+1} u_{k, i}^{t+1} L_{k, i}^{t+1}
$$

- Step 7f: Solve for $\left\{Y_{k, i}^{t+1}\right\}$ in the system

$$
\begin{gathered}
E_{k, i}^{t+1}=\mu_{k, i} E_{i}^{C, t+1}+\sum_{\ell=1}^{K}\left(\mu_{k, i} E_{\ell, i}^{V, t+1}+\left(1-\gamma_{\ell, i}\right) \nu_{\ell k, i} Y_{\ell, i}^{t+1}\right) . \\
Y_{k, o}^{t+1}=\sum_{i=1}^{N} \pi_{k, o i}^{t+1} E_{k, i}^{t+1} .
\end{gathered}
$$

- Step 7g: Compute $\left(\widetilde{w}_{k, i}^{t+1}\right)^{\prime}=\frac{\gamma_{k, i} Y_{k, i}^{t+1}}{\widetilde{L}_{k, i}^{t+1}}$

Step 8: Compute distance $\operatorname{dist}\left(\left\{\widetilde{w}_{k, i}^{t}\right\},\left\{\left(\widetilde{w}_{k, i}^{t}\right)^{\prime}\right\}\right)$

- Step 8b: Update $\widetilde{w}_{k, i}^{t}=\left(1-\lambda_{w}\right) \widetilde{w}_{k, i}^{t}+\lambda_{w}\left(\widetilde{w}_{k, i}^{t}\right)^{\prime} t=1, \ldots, T_{S S}$, for a small step size $\lambda_{w}$.

- Step 8c: At this point, we have a new series for $\left\{\widetilde{w}_{k, i}^{t}\right\}$ - go back to Step 3 until convergence of $\left\{\widetilde{w}_{k, i}^{t}\right\}$.

Step 9: Compute disposable income $\left\{I_{i}^{t}\right\}_{t=1}^{T_{S S}}$

$$
I_{i}^{t}=\sum_{\ell=1}^{K}\left(\gamma_{\ell, i} Y_{\ell, i}^{t}-E_{\ell, i}^{V, t}\right)
$$


Step 10: Compute $\left\{\left(\widehat{A}_{k, i}^{t}\right)^{\prime}\right\}$.

For $t=1, \ldots, T$ compute $\widehat{\widetilde{w}}_{k, i}^{t}=\frac{\widetilde{w}_{k, i}^{t}}{\widetilde{w}_{k, i}^{0}}$, obtain $\widehat{c}_{k, i}^{t}$ :

$$
\widehat{c}_{k, i}^{t}=\left(\widehat{\widetilde{w}}_{k, i}^{t}\right)^{\gamma_{k, i}} \prod_{\ell=1}^{K}\left(\widehat{P}_{\ell, i}^{I, t}\right)^{\left(1-\gamma_{k, i}\right) \nu_{k \ell, i}}
$$

and compute $\left(\widehat{A}_{k, i}^{t}\right)^{\prime}$ :

$$
\left(\widehat{A}_{k, i}^{t}\right)^{\prime}=\frac{\widehat{\pi}_{k, i i}^{t}}{\left(\widehat{P}_{k, i}^{I, t}\right)^{\lambda}}\left(\widehat{c}_{k, i}^{t}\right)^{\lambda}
$$

For $t \geq T+1$ set:

$$
\left(\widehat{A}_{k, i}^{t}\right)^{\prime}=\left(\widehat{A}_{k, i}^{T}\right)^{\prime}
$$

Feed outer loop with $\left\{\left(\widehat{A}_{k, i}^{T_{S S}}\right)^{\prime}\right\}$.

\section{Outer Loop: iteration on $\left\{N X_{i}^{t}\right\}$}

Step 0: Compute changes in trade costs $\left\{\widehat{d}_{k, o i}^{t}\right\}_{t=1}^{T}$ :

$$
\widehat{d}_{k, o i}^{t}=\left(\frac{\widehat{\pi}_{k, o o}^{t}}{\widehat{\pi}_{k, o i}^{t}}\right)^{1 / \lambda} \frac{\widehat{P}_{k, i}^{I, t}}{\widehat{P}_{k, o}^{I, t}}
$$

Set $\widehat{d}_{k, o i}^{t}=\widehat{d}_{k, o i}^{T}$ for $t>T$.

Step 1: Start with estimated state equilibrium at $t=0$. Remember that we used the normalization $\sum_{i=1}^{I} \sum_{k=1}^{K} Y_{k, i}=1$ during the estimation procedure. Change the normalization from $\sum_{i=1}^{I} \sum_{k=1}^{K} Y_{k, i}=1$ to $\sum_{i=1}^{I} E_{i}^{C}=1$. Nominal variables to be renormalized: $\left\{Y_{k, i}^{0}\right\},\left\{\widetilde{w}_{k, i}^{0}\right\},\left\{E_{i}^{C, 0}\right\},\left\{N X_{i}^{0}\right\}$.

Step 2: Compute $E_{i}^{C, t}=\frac{E_{i}^{C, 0}\left(\widehat{E}_{i}^{C, t}\right)_{\text {Data }}}{\sum_{i=1}^{N} E_{i}^{C, 0}\left(\widehat{E}_{i}^{C, t}\right)_{\text {Data }}}$ for $t=1, \ldots, T$ where $E_{i}^{C, 0}$ is aggregate consumption expenditure in the estimated steady state, and $\left(\widehat{E}_{i}^{C, t}\right)_{\text {Data }}$ comes from the data.

Step 3: Normalize $\widehat{\phi}_{U S}^{t}=1$ for $t=2, \ldots, T-1$. This yields

$$
R^{t+1}=\frac{E_{U S}^{C, t+1}}{\delta E_{U S}^{C, t}} \text { for } t=1, \ldots, T-1
$$

Obtain remaining shocks $\left\{\widehat{\phi}_{i}^{t}\right\}_{t=2}^{T}$ using

$$
\widehat{\phi}_{i}^{t+1}=\frac{E_{i}^{C, t+1}}{\delta E_{i}^{C, t} R^{t+1}} \text { for } t=1, \ldots, T-1
$$


Set $\widehat{\phi}_{i}^{t}=1$ for $t \geq T+2$. The value of $\widehat{\phi}_{i}^{T+1}$ will depend on $E_{i}^{C, T_{S S}}$ and will be recovered in Step 7 . Note: the value of $\widehat{\phi}_{i}^{t=1}$ does not matter. Individuals made decisions at $t=0$ assuming $\widehat{\phi}_{i}^{t=1}=1$, as the economy was assumed to be in steady state at $t=0$.

Step 4: Obtain $B_{i}^{0}$ with respect to the normalization $\sum_{i=1}^{I} E_{i}^{C}=1$. Equation (37) gives us:

$$
B_{i}^{0}=\frac{N X_{i}^{0}}{\left(1-\frac{1}{\delta}\right)}
$$

Step 5: Make initial guess for $N X_{i}^{T_{S S}}$ (with respect to the normalization $\sum_{i=1}^{I} E_{i}^{C}=1$ ) and for $\widehat{A}_{k, i}^{T_{S S}}=\widehat{A}_{k, i}^{T} \equiv \frac{A_{k, i}^{T}}{A_{k, i}^{0}}$

Step 6: Compute steady state equilibrium at $T_{S S}$, conditional on $N X_{i}^{T_{S S}}, \widehat{A}_{k, i}^{T_{S S}}$ and $\widehat{d}_{k, o i}^{T_{S S}}$.

- Step 6a: Notice that the steady-state algorithm uses the normalization $\sum_{i=1}^{I} \sum_{k=1}^{K} Y_{k, i}=1$. Normalize $N X_{i}^{T_{S S}}$ with respect to normalization $\sum_{i=1}^{I} \sum_{k=1}^{K} Y_{k, i}=1$. To perform such normalization, use revenue $\left\{Y_{k, i}^{T_{S S}}\right\}$ obtained in the initial steady state if this is the first outer loop iteration, otherwise use revenue $\left\{Y_{k, i}^{T_{S S}}\right\}$ obtained in Step 9 below.

- Step 6b: After computing the final steady state, change the normalization from $\sum_{i=1}^{I} \sum_{k=1}^{K} Y_{k, i}=$ 1 to $\sum_{i=1}^{I} E_{i}^{C}=1$ using $\left\{E_{i}^{C}\right\}$ obtained in Step 3a. Nominal variables to be renormalized: $\left\{Y_{k, i}^{T_{S S}}\right\},\left\{\widetilde{w}_{k, i}^{T_{S S}}\right\},\left\{E_{i}^{C, T_{S S}}\right\},\left\{N X_{i}^{T_{S S}}\right\}$.

Step 7: Set $E_{i}^{C, t}=\left\{\begin{array}{c}E_{i}^{C, 0}\left(\frac{E_{i}^{C, 2000+t}}{E_{i}^{C, 2000}}\right)_{\text {Data }} \text { for } t=0, \ldots, T \text {, as in Step } 0 \\ E_{i}^{C, T_{S S}} \text { for } t=T+1, \ldots, T_{S S}\end{array}\right.$

Note: $\widehat{\phi}_{i}^{t}=1$ for $t \geq T+2 \Rightarrow E_{i}^{C, t}=E_{i}^{C, T_{S S}}$ for at $t \geq T+1$ (Euler Equation)

Step 8: Compute $\widehat{\phi}_{i}^{T+1}$, imposing $\widehat{\phi}_{U S}^{T+1}=1$ :

$$
\begin{gathered}
\widehat{\phi}_{i}^{T+1}=\frac{E_{i}^{C, T_{S S}}}{\delta R^{T+1} E_{i}^{C, T}}, \\
R^{T+1}=\frac{E_{U S}^{C, T_{S S}}}{\delta E_{U S}^{C, T}} .
\end{gathered}
$$

We now have a full path for $\left\{\widehat{\phi}_{i}^{t}\right\}_{t=2}^{T_{S S}}$. 
Step 9: Solve for the out-of-steady-state dynamics conditional on aggregate expenditures $\left\{E_{i}^{C, t}\right\}_{t=0}^{T_{S S}}$, on preference shifters $\left\{\widehat{\phi}_{i}^{t}\right\}_{t=2}^{T_{S S}}$ and steady-state productivity shocks $\left\{\widehat{A}_{k, i}^{T_{S S}}\right\}$.

Step 10: Using the path for disposable income $\left\{I_{i}^{t}\right\}_{t=1}^{T_{S S}}$ obtained in Step 9 and equation (7) compute:

$$
\begin{gathered}
\left(N X_{i}^{t}\right)^{\prime}=I_{i}^{t}-E^{C, t} \text { for } 1 \leq t<T_{S S} \\
\left(N X_{i}^{T_{S S}}\right)^{\prime}=-\frac{1-\delta}{\delta} \frac{1}{\left(\prod_{\tau=1}^{T_{S S}-1}\left(R^{\tau}\right)^{-1}\right)}\left(B_{i}^{0}+\sum_{t=1}^{T_{S S}-1}\left(\prod_{\tau=1}^{t}\left(R^{\tau}\right)^{-1}\right)\left(N X_{i}^{t}\right)^{\prime}\right)
\end{gathered}
$$

Step 11: Compute

$$
\begin{gathered}
\operatorname{dist}\left(\left\{N X_{i}^{T_{S S}}\right\},\left\{\left(N X_{i}^{T_{S S}}\right)^{\prime}\right\}\right) \text { and } \\
\operatorname{dist}\left(\left\{\widehat{A}_{k, i}^{T_{S S}}\right\},\left\{\left(\widehat{A}_{k, i}^{T_{S S}}\right)^{\prime}\right\}\right),
\end{gathered}
$$

using the values of $\left\{\left(\widehat{A}_{k, i}^{T_{S S}}\right)^{\prime}\right\}$ obtained in Step 9.

Step 12: Update $N X_{i}^{T_{S S}}$

$$
N X_{i}^{T_{S S}}=\left(1-\lambda_{o}\right) N X_{i}^{T_{S S}}+\lambda_{o}\left(N X_{i}^{T_{S S}}\right)^{\prime}
$$

and $\widehat{A}_{k, i}^{T_{S S}}$

$$
\widehat{A}_{k, i}^{T_{S S}}=\left(1-\lambda_{A}\right) \widehat{A}_{k, i}^{T_{S S}}+\lambda_{A}\left(\widehat{A}_{k, i}^{T_{S S}}\right)^{\prime}
$$

for small step sizes $\lambda_{o}$ and $\lambda_{A}$.

Go back to Step 6 until convergence of $\left\{N X_{i}^{T_{S S}}\right\}$ and $\left\{\widehat{A}_{k, i}^{T_{S S}}\right\}$. 


\section{C.7 Parameter Estimates}

In this section, we display the complete set of parameter estimates.

Table A.1: Final Expenditure Shares $\mu_{k, i}$

\begin{tabular}{lcccccc}
\hline \hline Sector $\downarrow$ Country $\rightarrow$ & USA & China & Europe & Asia/Oceania & Americas & ROW \\
\hline Agr. & 0.01 & 0.12 & 0.02 & 0.01 & 0.02 & 0.09 \\
LT Manuf. & 0.03 & 0.02 & 0.04 & 0.03 & 0.04 & 0.03 \\
MT Manuf. & 0.05 & 0.11 & 0.08 & 0.07 & 0.1 & 0.11 \\
HT Manuf. & 0.1 & 0.15 & 0.11 & 0.1 & 0.11 & 0.1 \\
LT Serv. & 0.3 & 0.35 & 0.34 & 0.38 & 0.33 & 0.39 \\
HT Serv. & 0.51 & 0.25 & 0.41 & 0.41 & 0.4 & 0.29 \\
\hline \hline
\end{tabular}

Table A.2: Labor Shares in Production $\gamma_{k, i}$

\begin{tabular}{lcccccc}
\hline \hline Sector $\downarrow$ Country $\rightarrow$ & USA & China & Europe & Asia/Oceania & Americas & ROW \\
\hline Agr. & 0.45 & 0.58 & 0.56 & 0.54 & 0.62 & 0.67 \\
LT Manuf. & 0.37 & 0.25 & 0.32 & 0.35 & 0.28 & 0.27 \\
MT Manuf. & 0.33 & 0.28 & 0.31 & 0.37 & 0.32 & 0.28 \\
HT Manuf. & 0.39 & 0.24 & 0.33 & 0.32 & 0.31 & 0.25 \\
LT Serv. & 0.61 & 0.37 & 0.49 & 0.54 & 0.56 & 0.48 \\
HT Serv. & 0.62 & 0.55 & 0.63 & 0.67 & 0.67 & 0.68 \\
\hline \hline
\end{tabular}


Table A.3: Input-Output Table - Average Across Countries $\frac{1}{N} \sum_{i} \nu_{k \ell, i}$

\begin{tabular}{lcccccc}
\hline \hline User $\downarrow$ Supplier $\rightarrow$ & Agr. & LT Manuf. & MT Manuf. & HT Manuf. & LT Serv. & HT Serv. \\
\hline Agr. & 0.267 & 0.079 & 0.118 & 0.138 & 0.26 & 0.139 \\
& $(0.056)$ & $(0.019)$ & $(0.036)$ & $(0.029)$ & $(0.06)$ & $(0.07)$ \\
LT Manuf. & 0.195 & 0.376 & 0.043 & 0.081 & 0.223 & 0.082 \\
& $(0.047)$ & $(0.064)$ & $(0.009)$ & $(0.015)$ & $(0.041)$ & $(0.04)$ \\
MT Manuf. & 0.222 & 0.066 & 0.287 & 0.106 & 0.225 & 0.095 \\
& $(0.034)$ & $(0.017)$ & $(0.04)$ & $(0.022)$ & $(0.052)$ & $(0.044)$ \\
HT Manuf. & 0.022 & 0.157 & 0.067 & 0.463 & 0.184 & 0.106 \\
& $(0.018)$ & $(0.022)$ & $(0.015)$ & $(0.052)$ & $(0.044)$ & $0.05)$ \\
LT Serv. & 0.057 & 0.136 & 0.105 & 0.101 & 0.343 & 0.259 \\
& $(0.042)$ & $(0.03)$ & $(0.027)$ & $(0.034)$ & $(0.073)$ & $(0.11)$ \\
HT Serv. & 0.007 & 0.076 & 0.033 & 0.11 & 0.267 & 0.507 \\
& $(0.005)$ & $(0.023)$ & $(0.019)$ & $(0.071)$ & $(0.069)$ & $(0.171)$ \\
\hline \hline
\end{tabular}

Note: Standard Deviation of $\nu_{k \ell}$ across countries.

Table A.4: Mobility Costs Estimates $C_{k \ell}$

\begin{tabular}{lcccccc}
\hline \hline From $\downarrow /$ To $\rightarrow$ & Agr. & LT Manuf. & MT Manuf. & HT Manuf. & LT Serv. & HT Serv. \\
\hline Agriculture & 0 & 0.825 & 1.560 & 0.454 & 0.189 & 1.676 \\
LT Manufacturing & 0.414 & 0 & 0.005 & 0.000 & 0.799 & 2.034 \\
MT Manufacturing & 2.033 & 0.000 & 0 & 0.002 & 0.866 & 2.646 \\
HT Manufacturing & 0.015 & 0.001 & 0.003 & 0 & 0.276 & 0.917 \\
LT Services & 0.268 & 0.972 & 1.221 & 0.466 & 0 & 0.002 \\
HT Services & 0.790 & 1.826 & 2.150 & 1.201 & 0.004 & 0 \\
\hline \hline
\end{tabular}

Table A.5: Sector-Specific Utility and Variance Estimates $\eta_{k, i}, \sigma_{k, i}^{2}$

\begin{tabular}{lcccccc}
\hline \hline Sector $\downarrow /$ Country $\rightarrow$ & USA & China & Europe & Asia/Oceania & Americas & ROW \\
\hline Agriculture & 0 & 0 & 0 & 0 & 0 & 0 \\
LT Manuf. & -0.383 & -0.943 & -0.428 & -0.521 & -0.588 & -0.316 \\
MT Manuf. & 0.026 & -0.566 & -0.245 & -0.229 & -0.195 & -0.072 \\
HT Manuf. & -0.551 & -1.557 & -0.743 & -0.607 & -1.292 & -0.934 \\
LT Services & 0.085 & -0.454 & -0.382 & -0.236 & -0.405 & -0.588 \\
HT Services & -0.052 & -0.467 & -0.603 & -0.518 & -0.894 & -1.011 \\
\hline & & & & & & \\
$\sigma_{U S}^{2}$ & & & 0.727 & & \\
\hline \hline
\end{tabular}


Table A.6: Exogenous Death Rates Estimates $\chi_{k},=\chi_{i}+\chi_{k}$

\begin{tabular}{llc}
\hline \hline & USA & 0.026 \\
& China & 0.022 \\
Country & Europe & 0.054 \\
Component $\chi_{i}$ & Asia/Oceania & 0.021 \\
& Americas & 0.027 \\
& ROW & 0.020 \\
\hline \multirow{5}{*}{ Sector } & Agriculture & 0 \\
Component $\chi_{k}$ & Low Tech Man & 0.005 \\
& Med Tech Man & 0.014 \\
& Other Tech Man & 0.002 \\
& Hi Tech Services & 0.008 \\
& & -0.001 \\
\hline \hline
\end{tabular}

Table A.7: Unemployment Utility $b_{k, i}=b_{i}$

\begin{tabular}{ll}
\hline \hline USA & -14.4 \\
China & -12.7 \\
Europe & -7.1 \\
Asia/Oceania & -8.5 \\
Americas & -8.6 \\
ROW & -14.9 \\
\hline \hline
\end{tabular}

Table A.8: Vacancy Posting Costs Estimates $\widetilde{\kappa}_{k, i}$

\begin{tabular}{lcccccc}
\hline \hline Sector $\downarrow /$ Country $\rightarrow$ & USA & China & Europe & Asia/Oceania & Americas & ROW \\
\hline Agriculture & 0.539 & 0.450 & 0.451 & 0.664 & 0.451 & 0.452 \\
LT Manuf. & 0.632 & 0.810 & 0.681 & 0.865 & 0.809 & 0.583 \\
MT Manuf. & 0.472 & 0.615 & 0.549 & 0.660 & 0.540 & 0.447 \\
HT Manuf. & 0.698 & 0.984 & 0.821 & 0.922 & 1.076 & 0.830 \\
LT Services & 0.449 & 0.617 & 0.592 & 0.660 & 0.649 & 0.639 \\
HT Services & 0.511 & 0.674 & 0.672 & 0.840 & 0.872 & 0.813 \\
\hline \hline
\end{tabular}

